University of Windsor

Scholarship at UWindsor

\title{
Impact of product-design strategies on the operations of a closed-loop supply chain
}

\author{
Zhi Liu \\ Nanjing University of Aeronautics and Astronautics \\ Kevin Li \\ University of Windsor \\ Bang-Yi Li \\ Nanjing University of Aeronautics and Astronautics \\ Jun Huang \\ Hunan University \\ Juan Tang \\ Anhui Polytechnic University
}

Follow this and additional works at: https://scholar.uwindsor.ca/odettepub

Part of the Business Commons

\section{Recommended Citation}

Liu, Zhi; Li, Kevin; Li, Bang-Yi; Huang, Jun; and Tang, Juan. (2019). Impact of product-design strategies on the operations of a closed-loop supply chain. Transportation Research, Part E: Logistics and Transportation Review, 124, 75-91.

https://scholar.uwindsor.ca/odettepub/133

This Article is brought to you for free and open access by the Odette School of Business at Scholarship at UWindsor. It has been accepted for inclusion in Odette School of Business Publications by an authorized administrator of Scholarship at UWindsor. For more information, please contact scholarship@uwindsor.ca. 
Transportation Research, Part E: Logistics and Transportation Review, 124:

$75-91,2019$.

\title{
Impact of product-design strategies on the operations of a closed-loop supply chain
}

\author{
Zhi Liu ${ }^{\text {a, b, c }}$, Kevin W. Li ${ }^{\text {c, d }}$, Bang-Yi Li ${ }^{\text {a }}$, Jun Huang ${ }^{\text {e*, Juan Tang }}{ }^{\text {b }}$ \\ ${ }^{a}$ College of Economics and Management, Nanjing University of Aeronautics and Astronautics, Nanjing \\ 210016, China \\ ${ }^{\mathrm{b}}$ College of Management Engineering, Anhui Polytechnic University, Wuhu 241000, China \\ ${ }^{\mathrm{c}}$ Odette School of Business, University of Windsor, Windsor, Ontario, Canada N9B 3P4 \\ ${ }^{d}$ School of Economics and Management, Fuzhou University, Fuzhou, Fujian 350002, China \\ ${ }^{e}$ Business School, Hunan University, Changsha, Hunan 410082, China \\ * Corresponding author.
}

\section{Acknowledgments:}

The authors would like to acknowledge the financial support by the National Natural Science Foundation of China [grant numbers 71801003, 71572040, 71672055, 71671001, and 71771002] and a Discovery Grant funded by the Natural Sciences and Engineering Research Council of Canada (NSERC, grant \#: RGPIN-2018-05529).

\footnotetext{
* Corresponding author. Email: huangjun@hnu.edu.cn (Jun Huang).
} 


\title{
Impact of product-design strategies on the operations of a closed-loop supply chain
}

\begin{abstract}
This study investigates product design and its impact on the operations of a two-echelon closed-loop supply chain (CLSC). Research findings reveal that remanufacturing does not necessarily enhance the profitability of the supplier or the manufacturer, but adjusting product-design strategies helps to curb loss if profitability suffers. An environmental impact analysis identifies an interval for the base unit remanufacturing cost, within which remanufacturing is beneficial. We then obtain the condition under which the environmental impact can be mitigated by product design. These results shed insights for supply chain managers in their operational decisions and policy-makers in properly regulating remanufacturing activities.
\end{abstract}

Keywords: Product-design strategy; Closed-loop supply chain; Remanufacturing; Environmental impact

\section{Introduction}

The goal of remanufacturing is to recover residual values by disassembling, cleaning, and recovering end-of-use products, thereby improving resource utilization and reducing environmental footprints. The U.S. Environmental Protection Agency (1997) points out that remanufacturing can reduce raw material consumption and energy usage compared to regular manufacturing. Increased public awareness of and concerns about environmental impact and improved remanufacturing laws and regulations have resulted in rapid growth of the remanufacturing industry. In 2013, the annual sales of global remanufacturing industry were more than 150 billion U.S. dollars (Liu et al., 2017). Despite economic and environmental benefit of remanufacturing, some original equipment manufacturers (OEMs) remain reluctant to remanufacture their end-of-use products. Reasons that are cited include cannibalization of higher-margin new product sales and absence of proper infrastructure and expertise to collect and remanufacture used product profitably (Atasu et al., 2010; Ferguson and Toktay, 2006). On the other hand, some other OEMs choose to offer remanufactured products (e.g. Apple, Caterpillar, Cummins) for the sake of enhancing profitability or brand social image (McConocha and Speh, 1991) despite that the increased product variety makes supply chain operations more complicated (Fisher and Ittner, 1999; Wan et al., 2017). Many studies show that the remanufacturing decision of OEMs is contingent upon the corresponding cost savings (Liu et al., 2017; Xiong et al., 2013; Zheng et al., 2019), which are closely related to product design (Desai et al., 2001; Gu et al., 2015; Joshi and Gupta, 2019). If products are not properly designed for remanufacturing, it is difficult and costly to remanufacture them later on (Hatcher et al., 2011). Therefore, product design is a key factor that affects the efficiency of remanufacturing.

To make a product remanufacturing-friendly, proper product design has to be considered in many different aspects, ranging from material selection, to process designs and ease for disassembling and cleaning. Therefore, remanufacturing-oriented product design typically involves different design features such as modularity (Mukhopadhyay and Setoputro, 2005), interchangeability (Wu, 2013), possible disassembly (Wu, 2012), commonality (Subramanian et al., 2013), remanufacturability (Debo et al., 2005), and durability (Pangburn and Stavrulaki, 2014; Steeneck and Sarin, 2018). Generally 
speaking, remanufacturing-oriented product design interacts with other decisions such as collection (Atasu and Souza, 2013; Galbreth et al., 2013; Subramanian et al., 2013), remanufacturing (Reimann et al., 2019), and pricing strategies (Örsdemir et al., 2014; Wu, 2013). Existing studies show that a supply chain member, such as an OEM, may change its product-design strategy to deter its competitor from remanufacturing (Subramanian et al., 2013) or to attain win-win results for both parties (Wu, 2013). In addition, Gu et al. (2015) reveal that an OEM can adjust its product-design strategy to maximize its profits if it engages in remanufacturing. Therefore, product design proves to be an effective tool for a supply chain member to maximize its profit in a remanufacturing context.

The aforesaid studies assume that an integrated manufacturer is responsible for the design and production of a single product. But in reality, many products are designed and produced by a non-integrated manufacturer and its suppliers. For instance, PC manufacturers purchase CPUs from Intel or AMD and memory chips from other external chipmakers to make their final products. Most Chinese automakers rely on external suppliers for high-quality key components such as engines, gearboxes, and tires. It is apparent that the success of these manufacturers depends on their key component suppliers. Empirical evidence has revealed that an increasing number of OEMs are interested in motivating their suppliers to participate in their product development (Yan et al., 2018). In most cases, if a manufacturer engages in both producing new product and remanufacturing, the remanufactured product typically erodes demand for new final products, leading to reduced demand for components. For instance, as an engine producer, Cummins produces both new and remanufactured engines (here, the engine is a final product of Cummins). Its remanufactured engines are used to replace broken ones in faulty vehicles (Shi et al, 2016). It is apparent that Cummins' remanufactured engines compete with its new products, thereby reducing demand for engine parts such as bent axles. Another example is that automatic gearboxes used in many domestic automakers in China are bought from a Japanese firm, Aisin Group. As Chinese automakers start engaging in remanufacturing, demand for new automatic gearboxes from Aisin will be inevitably eroded. Similarly, Lenovo's battery remanufacturing from used computers can decrease new battery purchases from Sanyo (Huang and Wang, 2017). In these situations, the manufacturer is not only a partner but also a competitor of the supplier. As the designer and producer of the key component, the supplier may modify its component design and pricing to contain potential profit erosion ( $\mathrm{Wu}, 2013)$. The change in the component-design strategy presumably affects the design, production and remanufacturing of final products. Therefore, it is a worthy topic to investigate how the supplier and the manufacturer determine the remanufacturability level in their product design in a CLSC where the manufacturer collects and remanufactures used products. Based on existing literature (Debo et al., 2005; Wu, 2013), we define product (component) remanufacturability as the degree of difficulty that products (components) can be disassembled and recovered after use.

More specifically, we model and investigate a two-echelon CLSC consisting of a key component supplier and a non-integrated manufacturer. Hereafter, without causing confusion, these two members are referred to as the supplier and the manufacturer, respectively. At the beginning of a period, the supplier determines the remanufacturability level and wholesale price of the key component (component hereafter), and the manufacturer decides the remanufacturability level of the final product excluding the component (product hereafter). The manufacturer purchases new components from the supplier and, then produces and sells new final products to consumers. At the end of the period, used products (including components) are collected, disassembled, cleaned, inspected and remanufactured by the manufacturer. In line with existing literature, we assume 
that only the manufacturer engages in remanufacturing used products and the supplier does not remanufacture used components. The manufacturer relies heavily on the supplier for the key component in producing new final products, so the supplier is formulated as the leader and the manufacturer as the follower as depicted in Fig. 1.

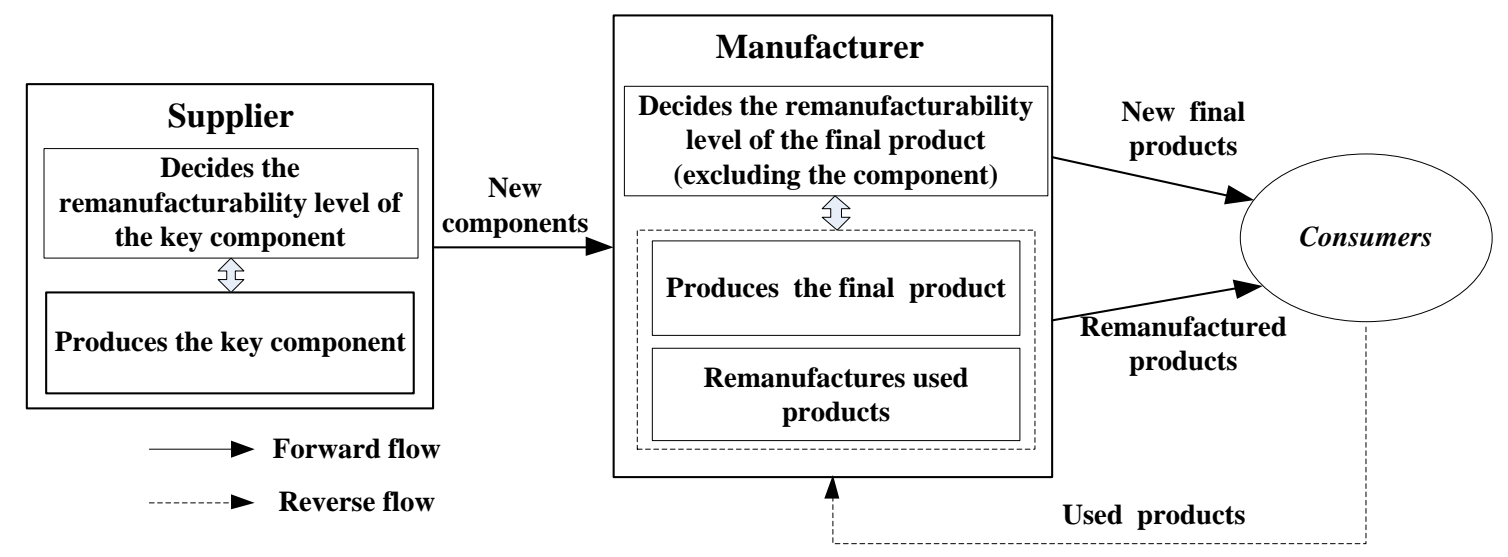

Fig 1. The decision-making process of the supplier and manufacturer in the two-echelon CLSC.

We address the following three main questions in this paper.

(1) How does the supplier's component-design strategy influence the manufacturer's decisions, such as the final product design and the quantity of new and remanufactured products?

(2) What is the manufacturer's optimal remanufacturing strategy when the base unit remanufacturing cost (without accounting for product design) changes? How do different model parameters influence the two CLSC members' optimal decisions, such as the wholesale price of the component, production quantities of new and remanufactured products, and the product-remanufacturability design choice?

(3) How do the two CLSC members' product-remanufacturability design strategies affect remanufacturing activity, economic and environmental performance of the CLSC?

The remainder of this paper is organized as follows. Section 2 reviews related literature. Section 3 introduces the model settings, assumptions and notations. Section 4 investigates the CLSC model in the general form, where the supplier is the channel leader and both members consider adjusting their product-design strategies. We then derive the equilibriums for three scenarios under which either the manufacturer, the supplier, or none of them adjusts the product remanufacturability level. Section 5 analyzes the manufacturer's remanufacturing strategy, equilibrium results, economic performance and environmental impact under different situations. Section 6 concludes the study with some remarks. For the sake of space, proofs are delegated to the Appendices.

\section{Literature review}

Extensive research has been conducted to examine different managerial decisions in CLSCs with remanufacturing such as strategic decisions, e.g., network design (Badri et al., 2017) and channel selection of CLCSs (Ma et al., 2017; Savaskan et al., 2004; Savaskan and Van Wassenhove, 2006), tactical and operational decisions (Choi, 2017; DeCroix, 2006; Gaur et al., 2017; Ma et al., 2017; Yenipazarli, 2016). Our research focuses on operational decisions such as product design and pricing decisions of the supply chain members. Therefore, our paper is closely related to two streams of literature: market segmentation and 
competition, and product design in CLSCs with remanufacturing.

The field of market segmentation and competition mainly consists of two streams. The first stream focuses on how the participants price new and remanufactured products in a non-competitive or a competitive context (Atasu et al., 2008; Ferrer and Swaminathan, 2010; Oraiopoulos et al., 2012). The second stream focuses on the decision interactions of multiple participants in the CLSC context. Research along this line typically assumes that consumer preference and demand are exogenous and explores the interaction between pricing or quantity decisions and other strategies such as product-design choices and collection strategies. For instance, game theoretic models are employed to investigate pricing/quantity/product-design decisions of an integrated manufacturer facing competition from IRs (Esenduran et al., 2017; Ferrer and Swaminathan, 2010; Majumder and Groenevelt, 2001; Örsdemir et al., 2014). In addition, some related literature studies economic and environmental consequences of remanufacturing. Earlier efforts conclude that remanufacturing generally enhances profitability and places less of burdens on environment (Atasu and Souza, 2013; Galbreth et al., 2013). But more recent research starts challenging this positive economic and environment profile of remanufacturing. For instance, Galbreth et al. (2013) show that remanufacturing can actually increase total raw material usage as the introduction of remanufactured products at a lower price increases overall demand. Xiong et al. (2013) point out that remanufacturing does not always benefit the members in a decentralized supply chain as it may lead to a win-lose, lose-lose or win-win situation contingent on the cost advantage of remanufacturing. Their results also indicate that a non-integrated manufacturer tends to produce more new products when new and remanufactured products are complements, thereby increasing the consumption of natural resources and energy, and aggravating environmental burdens. Gu et al. (2015) investigate environmental consequences of remanufacturing by defining a measure of environmental impact and reveal that production efficiency, green consumerism and production technology are not necessarily conducive to better environmental performance. Raz et al. (2017) investigate economic, environmental and social consequences of remanufacturing in a competitive setting, and find that remanufacturing worsens environmental impact but increases social welfare.

Within the stream of product design, with increased environmental consciousness and regulation, different product design strategies have been proposed such as product eco-design, design for disassembly, design for (recycling) remanufacturing (Joshi and Gupta, 2019; Steeneck and Sarin, 2018). Many studies have been conducted to help designers choose environmentally friendly design choices (Joshi and Gupta., 2019) and to investigate the impact of product design on the operations of supply chain (Hong et al., 2019). Another rich body of literature studies remanufacturing-oriented product design and its impact on operating CLSCs with remanufacturing. Researchers have noted that a significant issue is the interplay between product design and pricing (quantity) decisions in the CLSC context. For instance, Debo et al. (2005) address a joint pricing and production technology selection problem faced by a manufacturer who considers introducing a remanufacturable product in a market with heterogeneous consumers. By treating the level of interchangeability (disassemblability) as an endogenous variable, Wu (2013) investigates the OEM's product-design decision and the supply chain members' competitive pricing strategies. The result shows that the product-design strategy is effective for the OEM in competing with the remanufacturer, but this does not necessarily hurt the remanufacturer's profit. Qiang (2015) investigates the impact of remanufacturability design and consumers' perception of the remanufactured product on the manufacturers' profitability and 
market share in a two-period CLSC network. Shi et al. (2016) explore the effect of remanufacturable product design on market segmentation as well as product and trade-in prices by studying a two-stage profit-maximization problem. Subramanian et al. (2013) analyze how a firm's component commonality decision affects its product line and profit. Their result shows that the OEM will not introduce component commonality into the product line if this design can greatly reduce remanufacturing cost for third-party remanufacturers. Steeneck and Sarin (2018) model the production economics of remanufacturing at part-durability level for a leased product and examine how the profit margin of remanufactured products and recovery rate of leased products affect the optimal part durability. Reimann et al. (2019) reveal that the optimal strategy of process innovation (like design for disassembly) in a forward supply chain is not directly applicable to the CLSC, and their study shows that a decentralized supply chain is easier to engage in remanufacturing than an integrated supply chain when the process innovation is costly.

A general assumption in most existing literature is that a new product is designed and produced by an integrated manufacturer. But in reality, many products such as automobiles, personal computers, refrigerators, and air-conditioners are jointly designed and manufactured by non-integrated manufacturers and suppliers. In the automobile and personal computer industries, it is often the case that non-integrated manufacturers are heavily dependent on key component suppliers. Decisions of non-integrated manufacturers and suppliers interact with each other and influence the system efficiency. Up to now, limited research considers the role of a supplier when operational performance is examined for CLSCs. Xiong et al. (2013) and Xiong et al. (2016) recognize that previous studies (Aras et al., 2006; Jacobs and Subramanian, 2012) are carried out in a cooperative context, and there is no direct competition between a non-integrated manufacturer and a supplier. Their results demonstrate that the interaction between the supplier and non-integrated manufacturer can result in different economic and environmental outcomes in a co-opetitive context. Huang and Wang (2017) investigate the value of information sharing in supplier-remanufacturing and manufacturer-remanufacturing scenarios in a CLSC with a manufacturer, a supplier and a retailer. However, to the best of our knowledge, it remains open to explore the interplay between quantity decisions and the level of product remanufacturability chosen by the manufacturer and supplier in their product design. To close this gap in literature, we investigate how the CLSC members make decisions under product-remanufacturability design and further analyze the impact of product design on the economic and environmental performance of the CLSC.

The key contribution of this work is an explicit incorporation of the CLSC members' product-remanufacturability design into operational decisions in a two-echelon CLSC consisting of a supplier and a manufacturer. By formulating a general model to allow both the supplier and the manufacturer change their product remanufacturability in a supplier-dominated CLSC, we examine the interaction between the two CLSC members' product-design decisions, explore the impact of product design on the manufacturer's remanufacturing activity as well as operational performance of the CLSC. Our work reveals that remanufacturing is not always beneficial to the CLSC members or the environment, but adjusting product-design strategies can reduce the negative impact under certain conditions. More specially, in terms of the impact on profitability, when remanufacturing is successfully blocked by the supplier, or the manufacturer only remanufactures part of used product, the adjustment of the product-design strategy helps to contain the member's profit loss when it suffers a reduction owing to remanufacturing. When the manufacturer remanufactures all used products, the change of the product-design strategy improves 
both members' profitability. For the environmental impact, when remanufacturing is successfully blocked by the supplier, the change of the product-design strategy mitigates negative environmental impact due to remanufacturing, and this result remains true under one certain condition when the manufacturer remanufactures part of used products. When the manufacturer remanufactures all used products, adjusting product design will aggravate the environmental burdens. Our research thus complements the stream of remanufacturing literature with a focus on product design and market segmentation.

\section{Model settings, assumptions and notations}

This research has the following two features. First, new components are produced by the supplier, new and remanufactured products are produced by the manufacturer. Remanufactured products do not need to purchase new components. As such, remanufacturing is conceived to erode demand for both new products and the supplier's new components, and the manufacturer not only cooperates, but also competes with the supplier. Second, both the supplier and the manufacturer are concerned with their respective product-remanufacturability design, which directly affects the production cost of the new component and new final product as well as the remanufacturing cost. Below is a list of main assumptions concerning product design, cost structure, consumer preference, decision framework, environmental impact of new and remanufactured products.

Product-design Choice. We assume that the supplier and manufacturer can change their respective product remanufacturability in the cooperative and competitive context. $\gamma_{i}$ denotes that supplier's and manufacturer's choice of remanufacturability in their respective product, reflecting the remanufacturability level of $i$, where $i \in\{k, o\}$ represents the key component and final product. For the new product remanufacturability level $\gamma_{o}$, we assume that it is the manufacturer's product-design choice given the supplier's component-design decision. This assumption is reasonable given that the supplier is modeled as the leader in this model. Based on existing literature $(\mathrm{Wu}, 2013)$, we assume $\gamma_{i} \in[-1,1]$, where $-1 \leq \gamma_{i}<0$ $\left(0<\gamma_{i} \leq 1\right)$ denotes that the member adjusts its present product-design strategy to decrease (increase) the remanufacturability level, the used product is more (less) expensive to remanufacture. If the remanufacturability level equals 0 , the member will not adjust its current product-design strategy. Conceptually, if the supplier feels that the manufacturer's remanufacturing activity is a major threat for its component sales, it can adjust its component design to make it more expensive for the manufacturer to remanufacture used products, thereby mitigating cannibalization of demand for new components. Conversely, if the supplier can also benefit from the manufacturer's remanufacturing activity, it is then motivated to adjust its component design to make its more remanufacturing friendly. We first analyze how the supplier's component design and pricing decisions affect the manufacturer's product design and production decisions in the general model. Then we focus on the pricing and quantity decisions, economic and environmental performance under three special scenarios when the supplier, manufacturer, or none adjusts its product-design strategy for remanufacturing. We also assume that a used final product can be remanufactured once at most.

Cost Structure. A change in the product design incurs an additional fixed cost $F\left(\gamma_{i}\right)$ for the member before its production starts. Following Kim and Chhajed (2000), Mukhopadhyay and Setoputro (2005) and Biswas et al. (2018), we define $F\left(\gamma_{i}\right)$ as a quadratic function of $\gamma_{i}: F\left(\gamma_{i}\right)=\beta_{i} \gamma_{i}^{2} / 2$, where $\beta_{i}$ is the sensitivity parameter of the additional fixed cost with respect to $\gamma_{i}$. Remanufacturability has a great impact on the unit production cost of new and remanufactured 
products. Denote the unit production cost of remanufactured products by $c_{r}-\sum_{i=k, o} \Delta_{i} \gamma_{i}$. It is clear that this cost is reduced to $c_{r}$ when none of the supplier or manufacturer changes its current product-design strategy, i.e., $\gamma_{i}=0, i \in\{k, o\}$. For simplicity, we refer to $c_{r}$ as the base unit remanufacturing cost. $0<\Delta_{i}<1$ represents the impact factor of product-remanufacturability design on remanufacturing cost. As for the impact of remanufacturability design on the unit production cost of a new component (a final product), following Debo et al. (2005) and Gu et al. (2015), we assume that a higher remanufacturability level requires a higher unit production cost for a new component (final product) due to the use of better materials and more customized production processes. As such, we define the unit production cost of a new component (final product) by $c_{i}+\eta_{i} \gamma_{i}$, where $0<\eta_{i}<1$ is the impact factor of product-remanufacturability design on its unit production cost, $c_{i}$ is the base unit production cost of a key component and a final product (excluding the component) under the current product-design strategy, and $c_{i}+\eta_{i} \gamma_{i}>0$. Following Xiong et al. (2013) and Xiong et al. (2016), we assume that each final product requires one key component and the base unit production cost of new final products is thus $\omega+c_{o}$, where $\omega$ is the unit wholesale price of the key component, and $c_{r}<\omega+c_{o}$ (Giutini and Gaudette, 2003; Choi et al., 2013).

Consumer Preference. Remanufactured products can have as good quality as new products; however, latest empirical evidence and experimental results show that consumers' valuation of remanufactured products is generally lower (Agrawal et al., 2015; Guide $\mathrm{Jr}$ and $\mathrm{Li}, 2010$ ). We assume that the potential market size is $Q$, and consumer's willingness-to-pay $\theta$ for the new product is heterogeneous and uniformly distributed on $[0, Q] . \delta \theta$ is the consumer's willingness-to-pay for the remanufactured product, $\delta \in(0,1)$. Let $p_{n}$ and $p_{r}$ denote the prices of new and remanufactured products, respectively. The net utility of type $\theta$ consumers from getting a new product, remanufactured product and no product is $\theta-p_{n}, \delta \theta-p_{r}$ and 0 , respectively. In a given period, consumers buy a product based on the net utility that they can derive from the purchase during the period (Debo et al., 2005; Li and Chen, 2018). Following Johnson and Myatt (2006), consumer choices lead to a new product price $p_{n}=Q-q_{n}-\delta q_{r}$, and remanufactured product price $p_{r}=\delta\left(Q-q_{n}-q_{r}\right)$, where $q_{n}$ and $q_{r}$ denote the production quantity of new and remanufactured products, respectively. For simplicity, we normalize $Q$ to 1 (Ma et al., 2017 ; Taleizadeh et al., 2017). At the same time, we assume that all new products can be sold, and the volume of sales is larger than 0 when the manufacturer sells new products at the price of the unit production cost; therefore, $1-\omega-c_{o}+c_{r}-\delta>0$.

Decision Framework. All decisions are considered in a steady state period (Örsdemir et al., 2014). In the general model, the supplier moves first to make its component-design decision and price the component, followed by the manufacturer's product-design and production quantity decisions for new and remanufactured products. Both members are risk-neutral and pursue profit-maximization, and have complete information (Cachon and Lariviere, 1999; Goyal and Netessine, 2007). Generally, only a portion of used products can be collected and remanufactured. However, to keep our research focused, we consider the case that all used products are available for remanufacturing (Örsdemir et al., 2014; Reimann et al., 2019).

Environmental Impact. Researchers and policy-makers share the same view that the environmental impact of a remanufactured product is much smaller than that of a new product in its life cycle as remanufacturing consumes less raw materials and energy. Following the convention in literature (Agrawal et al., 2012; Atasu and Souza, 2013; Örsdemir et al., 2014), we assume that one unit of the new product and remanufactured product generates $e_{n}$ and $e_{r}$ environmental impact, respectively, where $e_{n}>e_{r}$. If the manufacturer produces $q_{n}$ units of new products and $q_{r}$ units of remanufactured 
products, the total environmental impact $E$ will be $e_{n} q_{n}+e_{r} q_{r}$.

Table 1 summarizes the notations used in this manuscript. In addition, for a more concise description of equilibrium solutions, we denote $\xi_{0}=1+c_{k}-c_{o}, \xi_{1}=1-c_{k}-c_{o}, \xi_{2}=\delta-c_{r}$, and $\xi_{3}=\delta\left(c_{k}+c_{o}\right)-c_{r}$, where $\xi_{0}>\xi_{1}>\xi_{2}>0$.

Table 1. Parameters and decision variables.

\begin{tabular}{|c|c|c|}
\hline \multicolumn{2}{|c|}{ Symbol } & Definition \\
\hline Parameters & $\begin{array}{c}c_{r} \\
c_{k} \\
c_{o} \\
e_{n} / e_{r} \\
\delta \\
\beta_{i}\end{array}$ & $\begin{array}{l}\text { Base unit remanufacturing cost of a used product. } \\
\text { Base unit production cost of a new component. } \\
\text { Base unit production cost of a new final product excluding the component. } \\
\text { Unit environmental impact of a new/remanufactured product. } \\
\text { Consumer's value discount for a remanufactured product. } \\
\text { Sensitivity parameter of additional fixed cost with respect to the component ( } i=k) \\
\text { and final product ( } i=o \text { ) remanufacturability level. } \\
\text { Impact factor of the change in } i \text { 's remanufacturability level on the unit production } \\
\text { cost of the remanufactured product, } i \in\{k, o\} \text {. } \\
\text { Impact factor of the change in } i \text { 's remanufacturability level on the unit production } \\
\text { cost of the new component }(i=k) \text { and final product excluding the component }(i=o) \text {. }\end{array}$ \\
\hline $\begin{array}{c}\text { Decision and } \\
\text { auxiliary } \\
\text { variables }\end{array}$ & $\begin{array}{c}\gamma_{i} \\
\omega \\
q_{n} / q_{r} \\
p_{n} / p_{r} \\
E \\
\Pi_{j}^{h}\end{array}$ & $\begin{array}{l}\text { The supplier's and manufacturer's product-design strategy for their component }(i=k) \\
\text { and final product }(i=o) \text {, respectively, reflected as the remanufacturability level. } \\
\text { Unit wholesale price of a new component. } \\
\text { Production quantity of new/remanufactured final products. } \\
\text { Unit retail price of a new/remanufactured final product. } \\
\text { Total environmental impact. } \\
j=S(M) \text { signifies the supplier (manufacturer), } h=N(B) \text { indicates that none } \\
\text { (both) of } S \text { and } M \text { adjust(s) their current product-design strategy, and } h=S(M) \\
\text { means that } S(M) \text { adjusts its product-design choice. }\end{array}$ \\
\hline
\end{tabular}

\section{Equilibrium analysis}

\subsection{Equilibrium analysis of the general model}

In the general model, the supplier is the leader, and the manufacturer is the follower. This power structure is common in such industries as automobile and personal computer. Based on the assumptions in Section 3, the profit functions of the supplier and manufacturer can be formulated as:

$$
\begin{gathered}
\max _{\gamma_{k}, \omega} \Pi_{S}^{B}=\left(\omega-c_{k}-\eta_{k} \gamma_{k}\right) q_{n}-\frac{1}{2} \beta_{k} \gamma_{k}^{2} \\
\max _{\gamma_{o}, q_{n}, q_{r}} \Pi_{M}^{B}=\left(p_{n}-\omega-c_{o}-\eta_{o} \gamma_{o}\right) q_{n}+\left(p_{r}-c_{r}+\gamma_{k} \Delta_{k}+\gamma_{o} \Delta_{o}\right) q_{r}-\frac{1}{2} \beta_{o} \gamma_{o}^{2} \\
\text { s.t. } 0 \leq q_{r} \leq q_{n}
\end{gathered}
$$

The constraint means that all used product can be collected and remanufactured by the manufacturer, but the quantity of available used products is constrained by the units of new products (Örsdemir et al., 2014; Reimann et al., 2019). Given the supplier's component wholesale price $(\omega)$ and remanufacturability level $\left(\gamma_{k}\right)$, the manufacturer's optimal response is given by Proposition 1.

Proposition 1. If $2 \beta_{o} \delta(1-\delta)-\left(\delta \eta_{o}^{2}+2 \delta \eta_{o} \Delta_{o}+\Delta_{o}^{2}\right)>0$, the manufacturer's optimal decisions in response to the supplier's 
$\omega$ and $\gamma_{k}$ are as follows.

Case $B$ - $A$ : If $\omega<\bar{\omega}_{1}$

$$
q_{n}^{B-A}=\frac{\beta_{o}\left(1-c_{o}-\omega\right)}{2 \beta_{o}-\eta_{o}^{2}}, \quad q_{r}^{B-A}=0, \text { and } \gamma_{o}^{B-A}=-\frac{\eta_{o}\left(1-c_{o}-\omega\right)}{2 \beta_{o}-\eta_{o}^{2}} ;
$$

Case $B$ - $B$ : If $\bar{\omega}_{1} \leq \omega \leq \bar{\omega}_{2}$

$$
\begin{gathered}
q_{n}^{B-B}=\frac{2 \delta \beta_{o}\left(1-c_{o}+c_{r}-\omega-\gamma_{k} \Delta_{k}-\delta\right)-\Delta_{o}\left(\eta_{o}\left(\delta+\gamma_{k} \Delta_{k}-c_{r}\right)+\Delta_{o}\left(1-c_{o}-\omega\right)\right)}{2\left(2 \beta_{o} \delta(1-\delta)-\left(\delta \eta_{o}^{2}+2 \delta \eta_{o} \Delta_{o}+\Delta_{o}^{2}\right)\right)} \\
q_{r}^{B-B}=\frac{2 \beta_{o}\left(\delta\left(c_{o}+\omega\right)+\gamma_{k} \Delta_{k}-c_{r}\right)-\eta_{o}^{2}\left(\delta+\gamma_{k} \Delta_{k}-c_{r}\right)-\eta_{o} \Delta_{o}\left(1-c_{o}-\omega\right)}{2\left(2 \beta_{o} \delta(1-\delta)-\left(\delta \eta_{o}^{2}+2 \delta \eta_{o} \Delta_{o}+\Delta_{o}^{2}\right)\right)}, \text { and } \gamma_{o}^{B-B}=\frac{\Delta_{o}\left(\delta\left(c_{o}+\omega\right)+\gamma_{k} \Delta_{k}-c_{r}\right)-\delta \eta_{o}\left(1-c_{o}+c_{r}-\omega-\gamma_{k} \Delta_{k}-\delta\right)}{2 \beta_{o} \delta(1-\delta)-\left(\delta \eta_{o}^{2}+2 \eta_{o} \delta \Delta_{o}+\Delta_{o}^{2}\right)}
\end{gathered}
$$

Case $B$ - $C$ : If $\omega>\bar{\omega}_{2}$

$$
q_{n}^{B-C}=q_{r}^{B-C}=\frac{\beta_{o}\left(1-c_{r}-\omega-c_{o}+\delta+\gamma_{k} \Delta_{k}\right)}{2 \beta_{o}(1+3 \delta)-\left(\Delta_{o}-\eta_{o}\right)^{2}} \text { and } \gamma_{o}^{B-C}=\frac{\left(1-c_{r}-\omega-c_{o}+\delta+\gamma_{k} \Delta_{k}\right)\left(\Delta_{o}-\eta_{o}\right)}{2 \beta_{o}(1+3 \delta)-\left(\Delta_{o}-\eta_{o}\right)^{2}} .
$$

Here, $\bar{\omega}_{1}=1-c_{o}-\frac{\left(2 \beta_{o}-\eta_{o}^{2}\right)\left(\delta+\gamma_{k} \Delta_{k}-c_{r}\right)}{2 \beta_{o} \delta+\eta_{o} \Delta_{o}}$ and $\bar{\omega}_{2}=1-c_{o}-\frac{\left(2 \beta_{o}(1+\delta)+\eta_{o}\left(\Delta_{o}-\eta_{o}\right)\right)\left(\delta+\gamma_{k} \Delta_{k}-c_{r}\right)}{4 \beta_{o} \delta-\Delta_{o}\left(\Delta_{o}-\eta_{o}\right)}$.

Proof. See Appendix A.

Proposition 1 shows how the manufacturer determines its product-design strategy and production quantities given the supplier's component wholesale price and remanufacturability choice. Rearranging $\omega<\bar{\omega}_{1}$ leads to $c_{r}>\delta+\gamma_{k} \Delta_{k}-\frac{\left(1-\omega-c_{o}\right)\left(2 \beta_{o} \delta+\Delta_{o} \eta_{o}\right)}{2 \beta_{o}-\eta_{o}^{2}}$, corresponding to the case that remanufacturing has no cost advantage compared to producing new products (case $B-A$ ). In this case, no remanufacturing activity occurs, the manufacturer lowers the product remanufacturability level for lower unit production cost of new products $\left(\gamma_{o}^{B-A}<0\right)$. When remanufacturing has moderate cost savings $\left(\bar{\omega}_{1} \leq \omega \leq \bar{\omega}_{2}\right)$, the profit from remanufacturing offsets the loss from cannibalization of new product sales, and the manufacturer remanufactures a portion of used products (referred to as partial remanufacturing). In this case (case $B$ - $B$ ), remanufactured products are substitutes for new products. Although there is remanufacturing activity, the remanufacturability level of the final product is not necessarily improved. An examination of the expression of $\gamma_{o}^{B-B}$ reveals that the manufacturer will enhance the final product remanufacturability level $\left(\gamma_{o}^{B-B}>0\right)$ if $\frac{\delta\left(c_{o}+\omega\right)+\gamma_{k} \Delta_{k}-c_{r}}{\delta+\gamma_{k} \Delta_{k}-c_{r}}>\frac{\eta_{o}(1-\delta)}{\eta_{o}+\Delta_{o}}$; otherwise, it will lower its product remanufacturability level $\left(\gamma_{o}^{B-B}<0\right)$. When remanufacturing has overwhelming cost savings $\left(\omega>\bar{\omega}_{2}\right)$, the manufacturer collects and remanufactures all used products (referred to as complete remanufacturing). In this case (case $B$ - $C$ ), remanufactured products are complements of new products. The choice of the product-design strategy depends on $\Delta_{o}-\eta_{o}$. If the product-design strategy has a greater impact on unit production cost of remanufactured products than that of new products $\left(\eta_{o}<\Delta_{o}\right)$, the product remanufacturability level is improved $\left(\gamma_{o}^{B-C}>0\right)$; otherwise, it is lowered $\left(\gamma_{o}^{B-C}<0\right)$.

Corollary 1. (1) The quantity of new products always decreases in $\omega$, but it is independent of $\gamma_{k}$ in case $B$ - $A$ and decreases (increases) in $\gamma_{k}$ in case $B-B(B-C)$; (2) The quantity of remanufactured products is independent of $\omega$ and $\gamma_{k}$ in case $B$ - $A$, increases (decreases) in $\omega$ in case $B-B(B-C)$, increases in $\gamma_{k}$ in cases $B-B$ and $B-C$.

\section{Proof. See Appendix B.}

Corollary 1 describes the manufacturer's optimal production quantity response given the supplier's wholesale pricing $\omega$ and component-design choice $\gamma_{k}$. It is natural that the quantity of new products always decreases in $\omega$ as an increased $\omega$ leads to a higher unit cost of the new product, thereby reducing its demand. In case $B$ - $A$, there is no remanufacturing activity 
and demand for remanufactured products remains zero. Due to given $\omega$ and no remanufacturing activity, the manufacturer's optimal response of new product production is independent of $\gamma_{k}$. When $\omega$ increases to the range of case $B$ - $B$, new and remanufactured products coexist and remanufactured products are substitutes for new products, so demand for remanufactured products increases in $\omega$. In addition, a higher component remanufacturability $\gamma_{k}$ enhances the relative cost advantage of remanufacturing and decreases (increases) demand for new (remanufactured) products. When $\omega$ falls within the range of case $B-C$, remanufactured products are complements of new products. Then, the production quantity of new and remanufactured products both decrease in $\omega$. As for $\gamma_{k}$, a higher value helps to lower remanufacturing cost, thereby enhancing demand for remanufactured products and the supplementary new products. As such, the quantities of new and remanufactured products both increase in $\gamma_{k}$.

Corollary 2. The product remanufacturability level increases (non-decreases) in $\omega\left(\gamma_{k}\right)$ in cases $B$ - $A$ and $B$ - $B$, decreases (increases) in $\omega\left(\gamma_{k}\right)$ in case $B-C$ if $\Delta_{o}>\eta_{o}$.

Proof. See Appendix C.

Corollary 2 shows the manufacturer's optimal product design response given the supplier's wholesale pricing $\omega$ and component-design choice $\gamma_{k}$ for its component. In case $B$ - $A$, as remanufacturing does not have sufficient cost advantage to cause any remanufacturing activity, the product remanufacturability level $\gamma_{o}$ is effectively decoupled from the supplier's component design strategy $\gamma_{k}$. On the other hand, as the component's wholesale price $\omega$ increases, the relative cost advantage of producing new products shrinks and the manufacturer will take a less aggressive strategy to reduce its product remanufacturability level (a negative $\gamma_{o}$ with a smaller magnitude). As such, $\gamma_{o}$ increases in the component's wholesale price $\omega$. When $\omega$ increases to the range in case $B$ - $B$ where remanufactured and new products are substitutes, the remanufacturing cost advantage is strengthened by higher component's wholesale price and remanufacturability level, stimulating the manufacturer to enhance its product remanufacturability level for remanufacturing. When the component's wholesale price further increases to case $B$ - $C$, the manufacturer's product-design strategy depends on its impact on the unit production cost of new and remanufactured products. If $\Delta_{o}>\eta_{o}$, the manufacturer enhances product remanufacturability level $\left(\gamma_{o}^{B-C}>0\right)$ and this level increases in $\gamma_{k}$ and decreases in $\omega$. Otherwise, the reverse holds.

Generally, given the manufacturer's optimal response functions, one can derive the equilibrium solutions of supplier's component pricing and remanufacturability design. However, given the complex interactions among the product design and other cost parameters in our general model, we cannot obtain the supplier's optimal decisions analytically. To address this issue, we derive equilibrium solutions for three special scenarios: $\gamma_{k}=0, \gamma_{o}=0$ (scenario $N$ ), $\gamma_{k}=0, \gamma_{o} \neq 0 \quad($ scenario $M$ ) and $\gamma_{k} \neq 0, \gamma_{o}=0$ (scenario $S$ ), and examine their managerial insights. Then, by numerical analysis in Section 5, we explore the robustness of these managerial insights in the general scenario, $\gamma_{k} \neq 0, \gamma_{o} \neq 0$ (scenario $B$ ). Hereafter, we use $h \in\{N, M, S, B\}$ to index different scenarios.

\subsection{Equilibrium solutions of three special scenarios}

\section{(1) Scenario $N$}

Scenario $N$ is the benchmark case when none of the two members adjusts its current product-design strategy and, hence, $\gamma_{k}=0, \gamma_{o}=0$. Based on Proposition 1 and the supplier's profit function, we can derive the optimal wholesale price of the 
component. Plugging it into the manufacturer's optimal decisions, the equilibrium solution under this scenario is given by:

Case $N-A: \omega^{N-A}=\frac{\xi_{0}}{2}, q_{n}^{N-A}=\frac{\xi_{1}}{4}$, and $q_{r}^{N-A}=0$, if $c_{r}>c_{r 4}^{N}$;

Case $N-B-1: \omega^{N-B-1}=\frac{c_{r}}{\delta}-c_{o}, q_{n}^{N-B-1}=\frac{\xi_{2}}{2 \delta}$, and $q_{r}^{N-B-1}=0$, if $\max \left\{c_{r 2}^{N}, c_{r 3}^{N}\right\} \leq c_{r} \leq c_{r 4}^{N}$;

Case $N-B-2: \quad \omega^{N-B-2}=\frac{1}{2}\left(\xi_{0}-\xi_{2}\right), q_{n}^{N-B-2}=\frac{\xi_{1}-\xi_{2}}{4(1-\delta)}$, and $q_{r}^{N-B-2}=\frac{(1-\delta) \xi_{2}+\xi_{3}}{4 \delta(1-\delta)}$, if $c_{r 1}^{N} \leq c_{r}<c_{r 2}^{N}$;

Case $N-C: \omega^{N-C}=\frac{1}{2}\left(\xi_{0}+\xi_{2}\right), \quad q_{n}^{N-C}=q_{r}^{N-C}=\frac{\xi_{1}+\xi_{2}}{4(1+3 \delta)}$, if $0<c_{r}<\operatorname{com}\left\{c_{r 2}^{N}, c_{r 3}^{N}\right\}$.

\section{Proof. See Appendix D.}

The equilibrium solution under scenario $N$ shows that the supplier has four cases about its component pricing as per the base unit remanufacturing cost $c_{r}$. When $c_{r}$ is too large to make remanufacturing profitable, the manufacturer will not engage in remanufacturing (case $N-A$ ) and the supplier prices its component by considering both members' production costs. When $c_{r}$ reduces to the range of case $N-B-1$, remanufacturing has moderate cost advantage, the manufacturer is motivated to remanufacture. However, given that remanufactured products cannibalizes the component's demand, instead of competing with remanufactured products, a more profitable strategy for the supplier is to completely drive remanufactured products out of the market by aggressively pricing the component $\left(\omega^{N-B-1}<\omega^{N-A}\right)$ after accounting for the base unit production cost of new and remanufactured products. When $c_{r}$ further reduces to the range of case $N-B-2$, the significant cost advantage of remanufacturing induces the manufacturer to remanufacture part of used products and remanufactured products are substitutes for new products. When $c_{r}$ falls into the range of case $\mathrm{N}$-C the overwhelming remanufacturing cost advantage motivates the manufacturer to remanufacture all used products. In this case, the new and remanufactured products are complements, and the supplier and the manufacturer are engaged in a cooperative relationship. In cases $N-B-2$ and $N-C$, the supplier prices the component by considering the market relationship between new and remanufactured products, as well as their base unit production costs.

\section{(2) Scenario $M$}

Under scenario $M$, the manufacturer is concerned with the final product's remanufacturability, but the supplier does not change its current component-design strategy. Therefore, $\gamma_{k}=0, \gamma_{o} \neq 0$. Based on Proposition 1 and the supplier's profit function, the equilibrium solution is determined as follows.

Case $M-A: \quad \omega^{M-A}=\frac{\xi_{0}}{2}, \quad \gamma_{o}^{M-A}=-\frac{\eta_{o} \xi_{1}}{2\left(2 \beta_{o}-\eta_{o}^{2}\right)}, q_{n}^{M-A}=\frac{\beta_{o} \xi_{1}}{2\left(2 \beta_{o}-\eta_{o}^{2}\right)}$, and $q_{r}^{M-A}=0$, if $c_{r}>c_{r 4}^{M} ;$

Case $M-B-1: \quad \omega^{M-B-1}=1-c_{o}-\frac{\left(2 \beta_{o}-\eta_{o}^{2}\right) \xi_{2}}{2 \beta_{o} \delta+\eta_{o} \Delta_{o}}, \quad \gamma_{o}^{M-B-1}=-\frac{\eta_{o} \xi_{2}}{2 \beta_{o} \delta+\eta_{o} \Delta_{o}}, \quad q_{n}^{M-B-1}=\frac{\beta_{o} \xi_{2}}{2 \beta_{o} \delta+\eta_{o} \Delta_{o}}$, and $q_{r}^{M-B-1}=0$, if $\max \left\{c_{r 2}^{M}, c_{r 3}^{M}\right\} \leq c_{r} \leq c_{r 4}^{M}$

Case $\quad M-B-2: \quad \omega^{M-B-2}=\frac{1}{2}\left(\xi_{0}-\frac{\left(2 \beta_{o} \delta+\eta_{o} \Delta_{o}\right) \xi_{2}}{2 \beta_{o} \delta-\Delta_{o}^{2}}\right) \quad, \quad \gamma_{o}^{M-B-2}=\frac{1}{2}\left(\frac{\Delta_{o} \xi_{2}}{2 \beta_{o} \delta-\Delta_{o}^{2}}-\frac{\delta \eta_{o}\left(\xi_{1}-\xi_{2}\right)-\Delta_{o} \xi_{3}}{2 \beta_{o} \delta(1-\delta)-\left(\delta \eta_{o}^{2}+2 \delta \eta_{o} \Delta_{o}+\Delta_{o}^{2}\right)}\right)$, $q_{n}^{M-B-2}=\frac{\left(2 \beta_{o} \delta-\Delta_{o}^{2}\right) \xi_{1}-\left(2 \beta_{o} \delta+\Delta_{o} \eta_{o}\right) \xi_{2}}{4\left(2 \beta_{o} \delta(1-\delta)-\left(\delta \eta_{o}^{2}+2 \delta \eta_{o} \Delta_{o}+\Delta_{o}^{2}\right)\right)} \quad, \quad$ and $\quad q_{r}^{M-B-2}=\frac{1}{4}\left(\frac{2 \beta_{o} \xi_{2}}{2 \beta_{o} \delta-\Delta_{o}^{2}}-\frac{\eta_{o}\left(\Delta_{o} \xi_{1}+\eta_{o} \xi_{2}\right)-2 \beta_{o} \xi_{3}}{2 \beta_{o} \delta(1-\delta)-\left(\delta \eta_{o}^{2}+2 \delta \eta_{o} \Delta_{o}+\Delta_{o}^{2}\right)}\right) \quad$ if $c_{r 1}^{M} \leq c_{r} \leq c_{r 2}^{M}$

Case $M-C$ : 


$$
\omega^{M-C}=\frac{1}{2}\left(\xi_{0}+\xi_{2}\right), \quad \gamma_{o}^{M-C}=\frac{\left(\xi_{1}+\xi_{2}\right)\left(\Delta_{o}-\eta_{o}\right)}{2\left(2 \beta_{o}(1+3 \delta)-\left(\Delta_{o}-\eta_{o}\right)^{2}\right)}, \text { and } q_{n}^{M-C}=q_{r}^{M-C}=\frac{\beta_{o}\left(\xi_{1}+\xi_{2}\right)}{2\left(2 \beta_{o}(1+3 \delta)-\left(\Delta_{o}-\eta_{o}\right)^{2}\right)}, \text { if } 0<c_{r}<\operatorname{com}\left\{c_{r 2}^{M}, c_{r 3}^{M}\right\} \text {. }
$$

Proof. See Appendix E.

As the structure of the equilibrium solution shows that the supplier's decision under scenario $M$ is similar to that under scenario $N$, we will not elaborate further for this scenario.

(3) Scenario $S$

Under scenario $S$, the supplier is concerned with the component remanufacturability, but the manufacturer does not change its current product-design strategy. Therefore, $\gamma_{k} \neq 0, \gamma_{o}=0$. Based on Proposition 1 and the supplier's profit function, its optimal solution is unique if $4 \beta_{k}(1-\delta)-\left(\eta_{k}+\Delta_{k}\right)^{2}>0$. The equilibrium solution is then given as follows:

Case $S-A: \omega^{S-A}=\frac{2 \beta_{k} \xi_{0}-\eta_{k}^{2}\left(1-c_{o}\right)}{4 \beta_{k}-\eta_{k}^{2}}, \quad \gamma_{k}^{S-A}=-\frac{\eta_{k} \xi_{1}}{4 \beta_{k}-\eta_{k}^{2}}, \quad q_{n}^{S-A}=\frac{\beta_{k} \xi_{1}}{4 \beta_{k}-\eta_{k}^{2}}$, and $q_{r}^{S-A}=0$, if $c_{r}>c_{r 4}^{S}$;

Case $\quad S-B-1: \quad \omega^{S-B-1}=\frac{2 \beta_{k} \delta\left(c_{r}-\delta c_{o}\right)+\eta_{k} \Delta_{k}\left(c_{r}+\delta-2 \delta c_{o}\right)+\Delta_{k}^{2} \xi_{0}}{2\left(\beta_{k} \delta^{2}+\Delta_{k}\left(\eta_{k} \delta+\Delta_{k}\right)\right)} \quad, \quad \gamma_{k}^{S-B-1}=-\frac{\left(\delta \eta_{k}+\Delta_{k}\right) \xi_{2}+\Delta_{k} \xi_{3}}{2\left(\beta_{k} \delta^{2}+\Delta_{k}\left(\delta \eta_{k}+\Delta_{k}\right)\right)} \quad$, $q_{n}^{S-B-1}=\frac{\left(2 \beta_{k} \delta+\eta_{k} \Delta_{k}\right) \xi_{2}+\Delta_{k}^{2} \xi_{1}}{4\left(\beta_{k} \delta^{2}+\Delta_{k}\left(\eta_{k} \delta+\Delta_{k}\right)\right)}$, and $q_{r}^{S-B-1}=0$, if $\max \left\{c_{r 2}^{S}, c_{r 3}^{S}\right\} \leq c_{r} \leq c_{r 4}^{S}$;

Case

$S-B-2:$

$$
\omega^{S-B-2}=c_{k}+\frac{\left(2 \beta_{k}(1-\delta)-\eta_{k}\left(\eta_{k}+\Delta_{k}\right)\right)\left(\xi_{1}-\xi_{2}\right)}{4 \beta_{k}(1-\delta)-\left(\eta_{k}+\Delta_{k}\right)^{2}} \quad,
$$$$
\gamma_{k}^{S-B-2}=-\frac{\left(\xi_{1}-\xi_{2}\right)\left(\eta_{k}+\Delta_{k}\right)}{4 \beta_{k}(1-\delta)-\left(\eta_{k}+\Delta_{k}\right)^{2}}
$$

$q_{n}^{S-B-2}=\frac{\beta_{k}\left(\xi_{1}-\xi_{2}\right)}{4 \beta_{k}(1-\delta)-\left(\eta_{k}+\Delta_{k}\right)^{2}}$, and $q_{r}^{S-B-2}=\frac{\left(2 \beta_{k}(2-\delta)-\eta_{k}\left(\eta_{k}+\Delta_{k}\right)\right) \xi_{2}-\left(2 \beta_{k} \delta+\Delta_{k}\left(\eta_{k}+\Delta_{k}\right) \xi_{1}\right)}{2 \delta\left(4 \beta_{k}(1-\delta)-\left(\eta_{k}+\Delta_{k}\right)^{2}\right)}$, if $c_{r 1}^{S} \leq c_{r} \leq c_{r 2}^{S}$;

Case $\quad S-C: \quad \omega^{S-C}=c_{k}+\frac{\left(2 \beta_{k}(1+3 \delta)+\eta_{k}\left(\Delta_{k}-\eta_{k}\right)\right)\left(\xi_{1}+\xi_{2}\right)}{4 \beta_{k}(1+3 \delta)-\left(\Delta_{k}-\eta_{k}\right)^{2}} \quad, \quad \gamma_{k}^{S-C}=\frac{\left(\Delta_{k}-\eta_{k}\right)\left(\xi_{1}+\xi_{2}\right)}{4 \beta_{k}(1+3 \delta)-\left(\Delta_{k}-\eta_{k}\right)^{2}} \quad, \quad$ and $q_{n}^{S-C}=q_{r}^{S-C}=\frac{\beta_{k}\left(\xi_{1}+\xi_{2}\right)}{4 \beta_{k}(1+3 \delta)-\left(\Delta_{k}-\eta_{k}\right)^{2}}$, if $0<c_{r}<\operatorname{com}\left\{c_{r 2}^{S}, c_{r 3}^{S}\right\}$

Proof. See Appendix F.

Similarly, the equilibrium solution illustrates that the supplier's pricing decision for the component under scenario $S$ resembles that under scenario $N$. The supplier's component-design decision is contingent upon the manufacturer's remanufacturing activity. When the manufacturer does not remanufacture at all in cases $S$ - $A$ and $S$ - $B$ - 1 or remanufactures part of used products in case $S-B-2$, lowering the component remanufacturability level not only reduces the component's unit production cost, but also weakens market cannibalization from remanufactured products. Therefore, the supplier takes a negative attitude towards remanufacturability design of its component $\left(\gamma_{k}^{S-A}<0 ; \gamma_{k}^{S-B-1}<0 ; \gamma_{k}^{S-B-2}<0\right)$. When the manufacturer remanufactures all used products in case $S$ - $C$, the new and remanufactured products are complements and the supplier's optimal component-design strategy is determined by its impact on the base unit production cost of new components and remanufactured products. If it has a greater impact on the base unit production cost of remanufactured products than that of new components $\left(\Delta_{k}>\eta_{k}\right)$, improving the component remanufacturability level $\left(\gamma_{k}^{S-C}>0\right)$ enhances the overwhelming remanufacturing cost advantage and brings in more remanufacturing profit for both members. The increase in remanufacturing profit surpasses the increased cost owing to the component-remanufacturability design. Otherwise, it is in the supplier interest to reduce the component remanufacturability level $\left(\gamma_{k}^{S-C}<0\right)$.

\section{Result analysis}


This section starts with a further discussion of the manufacturer's remanufacturing strategy and, then, characterizes the equilibrium decisions from the product-design and production perspectives. Next, a comparison is conducted for these equilibrium decisions under different scenarios. Subsequently, we analyze the supplier's and the manufacturer's profit with respect to the base unit remanufacturing cost $c_{r}$. Finally, we assess the total environmental impact of new and remanufactured products.

\subsection{The manufacturer's remanufacturing strategy}

By examining the equilibrium solutions under the three special scenarios, we find that the basic structure and property remain the same except that the thresholds of different cases may slightly shift for the three scenarios. The manufacturer's remanufacturing strategy depends not only on the base unit remanufacturing cost, but also on consumer's willingness-to-pay for remanufactured product. This result is presented in Proposition 2 and visually illustrated in Fig. 2.

Proposition 2. Under scenario $h \in\{N, M, S\}$, if $\delta<\bar{\delta}_{h}$, the manufacturer has three possible remanufacturing strategies: no, partial, and complete remanufacturing; otherwise, it has only two optimal choices: no and complete remanufacturing.

Proof. See Appendix G.

Proposition 2 indicates that consumer's willingness-to-pay for remanufactured products has a significant impact on the equilibrium solution structure. A higher consumer's willingness-to-pay for remanufactured products implies a higher relative cost advantage for remanufacturing compared to producing new products, making it easier for the manufacturer to break the barrier for committing to remanufacturing. At a higher willingness-to-pay level, the manufacturer will skip the partial remanufacturing strategy and jump from no to complete remanufacturing at a higher base unit remanufacturing cost $c_{r}$. This result is clearly shown in Fig. 2, where the manufacturer's remanufacturing strategies differ at two willingness-to-pay levels $\delta=0.72$ and $\delta=0.55$. The boundary value of $c_{r}$ at $\delta=0.72$ is higher than that at $\delta=0.55$ for the manufacturer to shift its remanufacturing strategy under all of the four scenarios, and the manufacturer will skip the partial remanufacturing strategy at a higher willingness-to-pay level $\delta=0.72$.

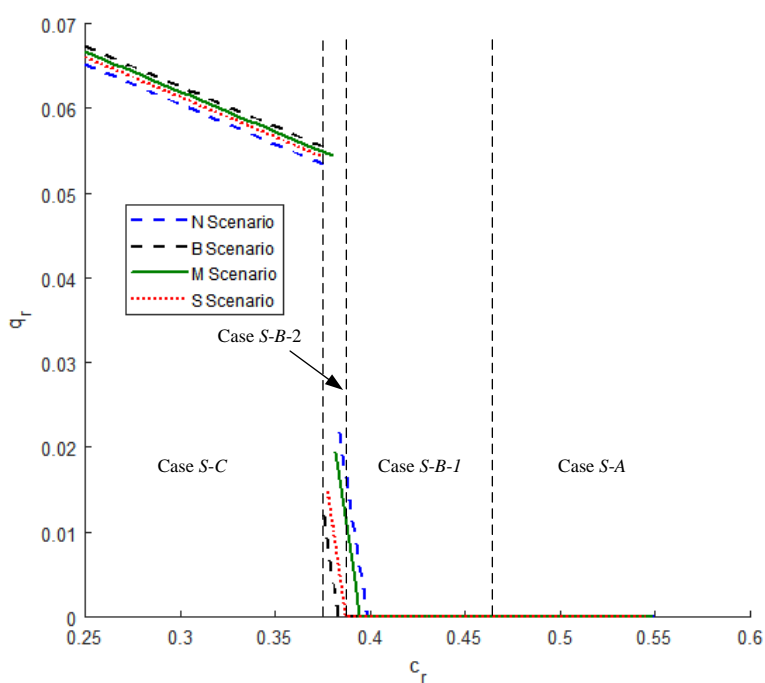

(a) $\delta=0.55$

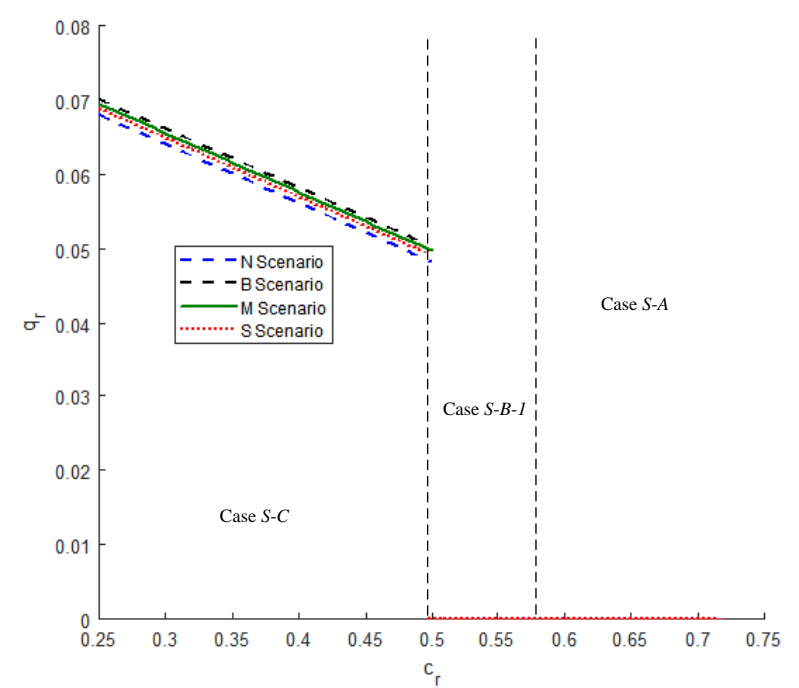

(b) $\delta=0.72$

Fig 2. The manufacturer's remanufacturing strategies $\left(\beta_{k}=\beta_{o}=1.2, \quad c_{k}=0.4, c_{o}=0.2, \Delta_{o}=0.07, \Delta_{k}=0.15, \eta_{o}=0.14, \eta_{k}=0.24\right)$

As partial remanufacturing is a more common remanufacturing strategy in reality, our discussions next shall focus on the 
case when $\delta<\bar{\delta}_{h}$.

\subsection{Characterization of equilibrium decisions}

\subsubsection{Characterization of equilibrium product-design decisions}

Result 1 characterizes the impact of different cost parameters on the product-design decisions under scenarios $M$ and $S$, respectively. In this table, a " 0 " at the intersection of $c_{r}$ and $\gamma_{i}^{h-A}$ indicates that the component (product) remanufacturability level is independent of $c_{r}$, a " + " at the intersection of $c_{k}$ and $\gamma_{i}^{h-B-2}$ means that $\gamma_{i}^{h-B-2}$ increases in $c_{k}$, a "-" at the intersection of $c_{r}$ and $\gamma_{i}^{h-B-2}$ signifies that $\gamma_{i}^{h-B-2}$ decreases in $c_{r}$, and a " \pm " at the intersection of $c_{o}$ and $\gamma_{i}^{h-C}$ shows an uncertain relationship between $c_{o}$ and $\gamma_{i}^{h-C}$. Symbols in a bracket such as " $(0,-)$ " at the intersection of $c_{k}$ and $\gamma_{i}^{h-B-1}$ shows the corresponding relationship under scenarios $M$ and $S$, respectively, meaning that $\gamma_{o}^{M-B-1}$ is independent of $c_{k}$, but $\gamma_{k}^{S-B-1}$ decreases in $c_{k}$. The other entries in Table 2 can be interpreted in the same fashion.

Result 1. The following result holds.

Table 2. The impact of parameters $c_{r}, c_{k}$ and $c_{o}$ on the remanufacturability level of the component and product

\begin{tabular}{ccccc} 
Parameter & $\gamma_{i}^{h-A}$ & $\gamma_{i}^{h-B-1}$ & $\gamma_{i}^{h-B-2}$ & $\gamma_{i}^{h-C}$ \\
\hline$c_{r}$ & 0 & + & - & \pm \\
$c_{k}$ & + & $(0,-)$ & + & \pm \\
$c_{o}$ & + & $(0,-)$ & + & \pm \\
\hline
\end{tabular}

Proof. See Appendix H.

More specifically, when remanufacturing has no cost advantage compared to producing new products (case $h-A)$, no remanufacturing takes place. In this case, the remanufacturability design of the component (product) takes a negative value to lower the unit cost of the new product, and it is independent of the base unit remanufacturing cost. On the other hand, an increase in $c_{o}$ and $c_{k}$ hurts the profit margin and demand for new products. By increasing the negative remanufacturability level of the component (final product), the savings on the fixed cost is more than enough to offset the profit loss due to lower profit margin and demand. Therefore, $\gamma_{i}^{h-A}$ increases in $c_{o}$ and $c_{k}$ in case $h-A$.

When $c_{r}$ decreases to the region in case $h-B-1$, remanufacturing has moderate cost advantage and the manufacturer has motivation to remanufacture. However, the supplier under scenario $M$ (scenario $S$ ) in this case takes an aggressive pricing (and component-design strategy) to block remanufacturing. While remanufacturing is blocked, the manufacturer under scenario $M$ continues to lower product remanufacturability level $\left(\gamma_{o}^{M-B-1}<\gamma_{o}^{M-A}<0\right.$, see the solid green line in Fig. 3(d)). Within this range, a higher $c_{r}$ indicates more cost advantage of producing new products, allowing the manufacturer under scenario $M$ (the supplier under scenario $S$ ) to take a less aggressive product-design strategy by increasing the product's negative remanufacturability level. On the other hand, the remanufacturing cost advantage is independent of $c_{k}\left(c_{o}\right)$ under scenario $M$ but shrinks as $c_{k}\left(c_{o}\right)$ increases under scenario $S$ and, then the manufacturer determines the product-design strategy regardless of $c_{k}\left(c_{o}\right)$ but the supplier has to take a more aggressive component-design strategy to effectively drive remanufactured products out of the market. Therefore, $\gamma_{i}^{h-B-1}(h \in\{M, S\}, i \in\{k, o\})$ increases in $c_{r}$, but non-increases in $c_{k}$ and $c_{o}$.

When the base unit remanufacturing cost $c_{r}$ further reduces to the range of case $h-B-2$, the manufacturer engages in partial remanufacturing and remanufactured products are substitutes for new products. As $c_{r}$ increases and $c_{k}\left(c_{o}\right)$ decreases, the remanufacturing cost advantage decreases, reducing demand for remanufactured products but enhancing demand for new 
products. This motivates the supplier (manufacturer) under scenario $S(M)$ to further undermine demand for remanufactured products by reducing the component (product) remanufacturability level. Therefore, $\gamma_{i}^{h-B-2}(h \in\{M, S\}, i \in\{k, o\})$ decreases in $c_{r}$, but increases in $c_{k}$ and $c_{o}$.

When the base unit remanufacturing cost $c_{r}$ falls in the range of case $h-C$, the supplier and the manufacturer are in a cooperative relationship. The impact of $c_{r}, c_{k}$ and $c_{o}$ on component (product) remanufacturability design depends on the relationship between the impact factor on unit production cost of remanufactured products $\Delta_{i}$ and that of new products $\eta_{i}(i \in\{k, o\})$. If $\Delta_{i}>\eta_{i}$, the remanufacturability level of the component (final product) is improved, $\gamma_{i}^{h-C}>0$. In this case, as $c_{r}$ increases, demand for remanufactured (subsequently, new) products will be reduced. Similarly, as $c_{k}$ or $c_{o}$ increases, demand for components and new (subsequently, remanufactured) products will also be reduced. These will dampen the participant's willingness to invest in positive component (product) remanufacturability design under scenario $S$ ( $M$ ). As such, $\gamma_{i}^{h-C}$ decreases in $c_{r}, c_{k}$, and $c_{o}$. Conversely, $\gamma_{i}^{h-C}$ increases in $c_{r}, c_{k}$, and $c_{o}$ if $\Delta_{i}<\eta_{i}$.

To visually illustrate how the equilibrium product-design decision varies with different parameters, we select $c_{r}$ as a representative parameter to plot the chart of $\gamma_{k}$ and $\gamma_{o}$ vs. $c_{r}$ in Fig. $3(d)$ by setting $\beta_{o}=\beta_{k}=1.2, \delta=0.5, c_{k}=0.4$, $c_{o}=0.2, \Delta_{k}=0.3, \Delta_{o}=0.25, \eta_{k}=0.12$, and $\eta_{o}=0.07$. The vertical dashed lines signify the boundaries between different cases for scenario $S$. The corresponding boundaries for scenarios $N, M$ and $B$ follow the same pattern as scenario $S$, but are slightly shifted.

Fig.3 $(d)$ not only confirms the analytical results in Table 2 (row $c_{r}$ ), but also demonstrates how $c_{r}$ influences the remanufacturability level of the component and product under the general scenario $B$ where $\gamma_{k} \neq 0, \gamma_{o} \neq 0$. Fig. $3(d)$ shows that scenario $B$ possesses the similar patterns of the remanufacturability levels of the component $\left(\gamma_{k}\right)$ and product $\left(\gamma_{o}\right)$ as well as the influence of $c_{r}$ on them as those under scenarios $S$ and $M$. Therefore, we infer that Result 1 can be extended to the general scenario $B$.

\subsubsection{Characterization of equilibrium production decisions}

Next, Result 2 demonstrates how equilibrium wholesale price and production quantity decisions are affected by $c_{r}, c_{k}$ and $c_{o}$ (Table 3). The notation in Result 2 is similar as that in Result 1 . Symbols in a bracket such as " $(0,0,+)$ " shows the corresponding relationship under scenario $h \in\{N, M, S\}$, respectively.

Result 2. The following result holds.

Table 3. The impact of parameters $c_{r}, c_{k}$ and $c_{o}$ on equilibrium wholesale price and production quantities

\begin{tabular}{|c|c|c|c|c|c|c|c|c|c|c|c|c|}
\hline Parameter & $\omega^{h-A}$ & $q_{n}^{h-A}$ & $q_{r}^{h-A}$ & $\omega^{h-B-1}$ & $q_{n}^{h-B-1}$ & $q_{r}^{h-B-1}$ & $\omega^{h-B-2}$ & $q_{n}^{h-B-2}$ & $q_{r}^{h-B-2}$ & $\omega^{h-C}$ & $q_{n}^{h-C}$ & $q_{r}^{h-C}$ \\
\hline$c_{r}$ & 0 & 0 & 0 & + & - & 0 & + & + & - & - & - & - \\
\hline$c_{k}$ & + & - & 0 & $(0,0,+)$ & $(0,0,-)$ & 0 & + & - & + & + & - & - \\
\hline$c_{o}$ & - & - & 0 & - & $(0,0,-)$ & 0 & - & - & + & - & - & - \\
\hline
\end{tabular}

Proof. See Appendix I.

Result 2 shows that the impacts of parameter $c_{k}\left(c_{o}\right)$ on the component wholesale price and the quantity of new products are the same in cases $h-A, h-B-2$ and $h-C$ for the three special scenarios, but their impacts on the quantity of remanufactured products depend on the relation between new and remanufactured products. Recall that $c_{k}$ is the base unit production cost of new components, so it is understandable that the component wholesale price increases in $c_{k}$. Consequently, 
when $c_{k}$ increases, the production cost of new products will be pushed higher owing to the heightened component price, thereby reducing demand for new products. When the manufacturer incurs a higher in-house production cost $c_{o}$, the supplier is motivated to lower the component's wholesale price to boost demand for components. Note that $\omega^{h-A}+c_{o}$ $\left(\omega^{h-B-1}+c_{o}, \omega^{h-B-2}+c_{o}\right)$ is the unit production cost of new products. When $c_{o}$ increases, the reduced component cost $\omega^{h-A}$ $\left(\omega^{h-B-1}, \omega^{h-B-2}\right)$ is insufficient to cover the heightened $c_{o}$, resulting in a higher unit production cost of and lower demand for new products. As for their impacts on the quantity of remanufactured products, when the manufacturer does not take up remanufacturing (case $h-A$ ), only new products are available in the market, the quantity of remanufactured products is always zero regardless of $c_{k}$ and $c_{o}$. When the manufacturer commits to partial (complete) remanufacturing in case $h-B$-2 (case $h-C$ ), remanufactured products are substitutes (complements) of new products, so it is understandable that the quantity of remanufactured product increases in $c_{k}$ and $c_{o}$ in case $h-B-2$ (in contrary to demand for new products), but decreases in them in case $h-C$ (consistent with demand for new products).

The impacts of $c_{r}$ on the equilibrium wholesale price and production quantities are also contingent upon the relation between new and remanufactured products in cases $h-A, h-B-2$ and $h-C$. In case $h-A$, only new products are available in the market, so $c_{r}$ is irrelevant and has no effect on the wholesale price or the production quantities. In case $h$ - $B$-2, remanufactured products are substitutes for new products, a higher $c_{r}$ reduces demand for remanufactured products but increases demand for new products. This allows the supplier to exert less effort in competing with remanufactured products, leading to a higher wholesale price for its component. In case $h$ - $C$, remanufactured products are complements of new products, as $c_{r}$ increases, the demand for remanufactured products (and, hence, new products) will decrease, leading the supplier to reduce component wholesale price to enhance its demand.

When remanufacturing has moderate cost advantage (case $h-B-1$ ), the manufacturer is motivated to remanufacture but is effectively blocked from remanufacturing, corresponding to the three 0 's under column $q_{r}^{h-B-1}$. For the supplier, its aim is to completely drive remanufactured products out of the market. When pricing the component is the only tool available for the supplier to achieve this aim (under scenarios $N$ and $M$ ), the supplier pegs on $c_{o}$ and $c_{r}$ regardless of $c_{k}$. However, when the supplier has two tools (aggressively pricing the component and lowering its remanufacturability level) to deter remanufacturing under scenario $S$, the combination of these two tools allows the supplier to act more rationally by increasing its wholesale price when $c_{k}$ increases. Meanwhile, a higher $c_{r}$ (a lower $c_{o}$ ) indicates that the supplier can have a less aggressive wholesale pricing for its components. Therefore, $\omega^{h-B-1}$ increases in $c_{r}$ and decreases in $c_{o}$ under the three scenarios, but $\omega^{N-B-1}$ and $\omega^{M-B-1}$ are independent of $c_{k}$ and $\omega^{S-B-1}$ increases in $c_{k}$. Consequently, $q_{n}^{h-B-1}$ decreases in $c_{r}$ under the three scenarios, but $q_{n}^{N-B-1}$ and $q_{n}^{M-B-1}$ are independent of $c_{k}$ and $q_{n}^{S-B-1}$ decreases in $c_{k}$. As for $c_{o}$, its impact on $q_{n}^{h-B-1}$ depends on the base unit production cost of new products $\omega^{h-B-1}+c_{o}$. As mentioned above, a higher $c_{o}$ leads to a lower $\omega^{h-B-1}$, and the increase in $c_{o}$ is exactly canceled by the decrease in $\omega^{h-B-1}$ under scenarios $N$ and $M$. But under scenario $S$, the increase in $c_{o}$ outpaces the decrease in $\omega^{S-B-1}$, resulting in a higher unit production cost of new products. Therefore, $q_{n}^{N-B-1}$ and $q_{n}^{M-B-1}$ are independent of $c_{o}$, but $q_{n}^{S-B-1}$ decreases in $c_{o}$.

Fig. 3 visually displays how the base remanufacturing cost parameter $c_{r}$ affects equilibrium wholesale price of the component, production quantities of new and remanufactured products. Fig. 3(a), $(b)$ and $(c)$ not only confirm the relationship 
between equilibrium variables and the base unit remanufacturing cost in Table 3 , but also show that it qualitatively holds under scenario $B$.

\subsubsection{Comparison of equilibrium decisions across different scenarios}

Next, we shall vertically compare equilibrium decisions between scenarios $M$ and $N$ as well as between scenarios $S$ and $N$ and obtain Result 3 as follows

Result 3. (1) The wholesale price of the component under scenario $M$ equals that under scenario $N$ in cases $h$ - $A$ and $h$ - $C$, but is higher (lower) than that in case $h-B-1(h-B-2)$; the wholesale price under scenario $S$ is lower (higher) than that under scenario $N$ in cases $h-A(h-B-1)$, is higher (lower) than that under scenario $N$ in case $h-B-2$ and $h-C$ if $\Delta_{k}>\eta_{k}$ (otherwise). (2) the quantities of new products under scenarios $M$ and $S$ are higher (lower) than those under scenario $N$ in cases $h-A, h-B-2$ and $h-C$ $(h-B-1)$; the quantities of remanufactured products under scenarios $M$ and $S$ are lower (higher) than those under scenario $N$ in case $h-B-2$ (in case $h-C$ ).

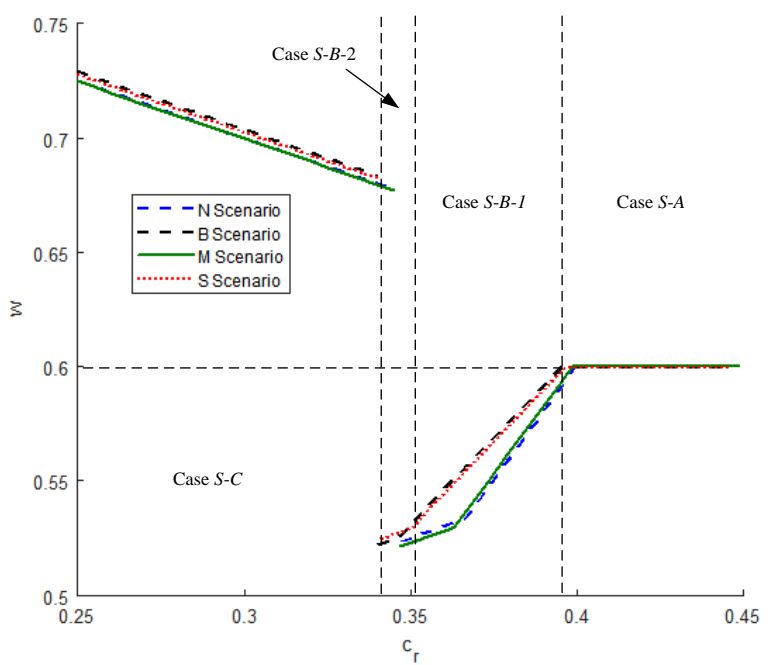

(a) The optimal wholesale price of key components

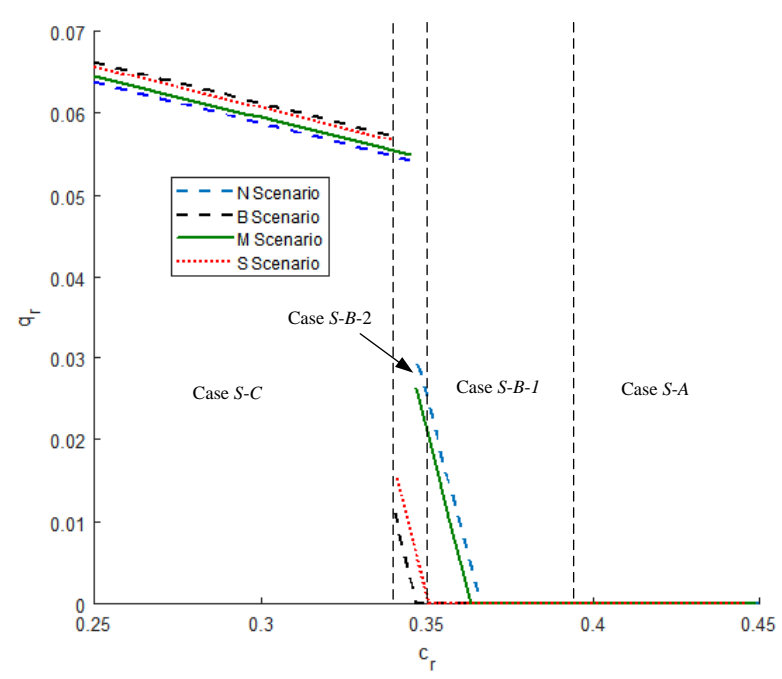

(c) The optimal quantity of remanufactured products

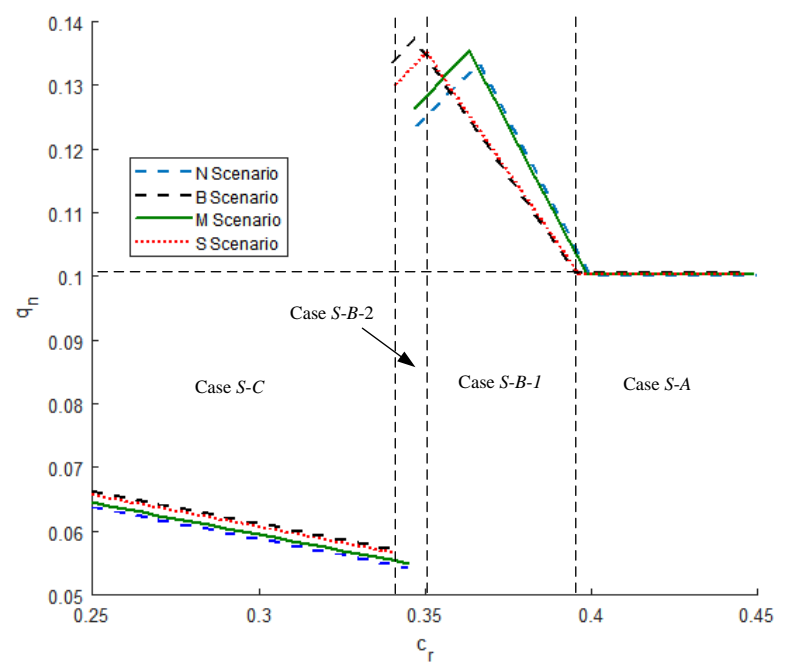

(b) The optimal quantity of new products

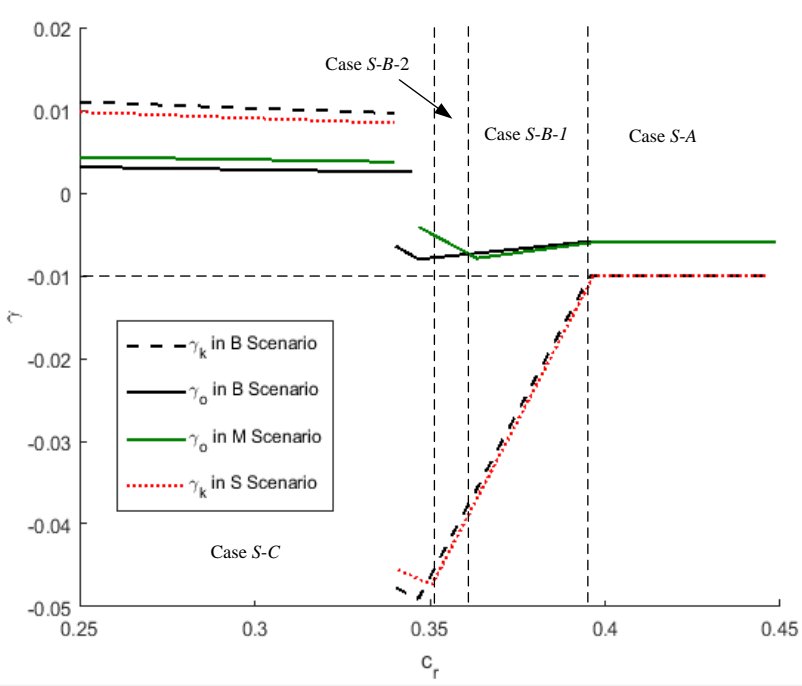

(d) The remanufacturability level of the component and product

Fig 3. Impact of $c_{r}$ on the equilibrium decisions

Proof. See Appendix J. 
When remanufacturing has no cost advantage (case $h-A$ ), the manufacturer will not commit to remanufacturing under any scenario. Compared to the base scenario $N$, the supplier under scenario $S$ lowers its component remanufacturability level to reduce its marginal cost, allowing it to charge a lower wholesale price. Although the manufacturer under scenario $M$ also lowers its product remanufacturability level to drive down its unit production cost, this adjustment affects only its own profit margin in producing new products and has no effect on the supplier's wholesale pricing decision. As such, we have $\omega^{S-A}<\omega^{N-A}, \omega^{M-A}=\omega^{N-A}$, and then $q_{n}^{S-A}>q_{n}^{N-A}$ and $q_{n}^{M-A}>q_{n}^{N-A}$. By following the aforesaid argument, as both members will lower their product remanufacturability levels as shown in Fig. 3(d), we can infer that the component wholesale price under the general scenario $B$ is lower than that under scenario $N$ and demand for new products is the highest under scenario $B$ compared to the other three special scenarios. If we zoom in the horizontal lines in case $h-A$ in Fig. 3(a) and $(b)$, we shall be able to confirm these relationships.

When $c_{r}$ decreases to the range in case $h-B-1$, the manufacturer has motivation to remanufacture but is effectively blocked from remanufacturing by the supplier. When all of the four scenarios fall within the range $B-1$, at the same $c_{r}$, the scenario with more tools to block remanufacturing will be able to price the component higher (leading to lower demand for components and new products). As such, as illustrated in Fig. 3( $a)$ and $(b)$, scenario $B$ has the highest wholesale price (the lowest demand for components and new products) as both members lower their product remanufacturability levels, followed by scenario $S$ (the second lowest demand for new products) where the supplier's lowering its component remanufacturability has a direct impact on the wholesale price and, then scenario $M$ (the second highest demand for new products) when the manufacturer's lowering its product remanufacturability has a secondary impact (deterring remanufacturing directly and enhancing new product production indirectly) on the wholesale price and, lastly, scenario $N$ (highest demand for new products). For the same reason, we note that a lower base unit remanufacturing cost $c_{r}$ (or a higher remanufacturing cost advantage) is needed for remanufacturing to arise as a profitable activity if the members adjust their product design. This is visually shown in Fig. 3(c), where scenario $B$ requires the lowest $c_{r}$, followed by a slightly higher $c_{r}$ in scenario $S$, then, an even higher $c_{r}$ in scenario $M$ and, lastly the highest $c_{r}$ in scenario $N$ for remanufacturing to arise in the equilibrium. Based on this line of thinking, we infer that the quantity of remanufactured products is smaller and the quantity of new products is larger under scenario $B$ than those under the other three scenarios when the manufacturer commits to partial remanufacturing (case $h-B$-2) (see Fig. 3(b) and (c)).

When all used products are remanufactured (case $h-C$ ), new and remanufactured products are complements. In this case, if $\Delta_{i}>\eta_{i}$, both the supplier under scenarios $S$ and $B$ and the manufacturer under scenarios $M$ and $B$ will enhance their product remanufacturability levels (see Fig. 3(d)). The supplier's positive component-design strategy pushes its production cost higher and allows it to charge a higher wholesale price for its component under scenarios $S$ and $B$ than that under scenario $N$. On the other hand, the downstream manufacturer's product design does not affect the supplier's component cost and, hence, the wholesale price of the component is the same for scenarios $M$ and $N$ (see case $h-C$ in Fig. 2(a)). Compared to the base scenario $N$, the two members' remanufacturing-friendly design facilitates remanufacturing and boosts demand for remanufactured products (subsequently, complementary new products) under scenarios $S, M$, and $B$. If $\Delta_{i}<\eta_{i}$, both the supplier under scenarios $S$ and $B$ and the manufacturer under scenarios $M$ and $B$ will reduce their product remanufacturability. The supplier's 
negative component-design strategy drives down its marginal production cost, leading to a lower wholesale price for its component under scenarios $S$ and $B$ than that under scenario $N$. For the same reason, the manufacturer's negative product-design choice under scenario $M$ does not impact the supplier's wholesale price decision for the component. Understandably, the negative product design $\left(\gamma_{i}^{h-C}<0\right)$ drives down the unit production cost of new products and boosts demand for new products (and, hence, complementary remanufactured products). Fig 2, 3(b) and 3(c) show that demand for remanufactured products (and, hence, new products) is the highest under scenario $B$ and is the lowest under scenario $N$ for the same $c_{r}$ in the common range in case $h-C$.

In summary, these analyses allow us to make inference on the relationship between the general scenario $B$ and the base scenario $N$. In case $h-B-1$, scenario $B$ is most effective in deterring the manufacturer from remanufacturing, followed by scenario $S$ when the supplier is concerned with component design and, then, scenario $M$ when the manufacturer adjusts product design, and lastly none is concerned under scenario $N$. In case $h-B-2$, demand for remanufactured (new) products is the smallest (largest) under scenario $B$ as it is the last to enter remanufacturing compared to the other three scenarios. In case $h$ - $C$, simultaneous changes of component and product remanufacturability choices boost demand for new and remanufactured products to the highest level under scenario $B$ compared to the other three special scenarios.

\subsection{Profit analysis}

Result 4 characterizes the profits of the supplier and manufacturer with respect to the base unit remanufacturing cost $c_{r}$. Result 4. In case $h-A(h \in\{N, M, S\})$, the supplier's and manufacturer's profits are independent of $c_{r}$. In cases $h-B$-1 and $h-B-2$, the supplier's profit increases in $c_{r}$, but the manufacturer's profit decreases in $c_{r}$. In case $h$ - $C$, both the supplier's and manufacturer's profits decrease in $c_{r}$.

\section{Proof. See Appendix K.}

Fig. 4 visually confirms the trend of profit changes with the value of $c_{r}$ as given in Result 4 . It is understandable that the two members' profits are independent of $c_{r}$ in case $h-A$ as no remanufacturing is involved. In this case, the two members can achieve the highest profitability under scenario $B$ compared to the other three special scenarios (see Fig. 4(a) and 4(b)) owing to the lowest production cost of new components and final products caused by their negative product-design strategies. When $c_{r}$ decreases to the range in case $h-B-1$, the manufacturer has motivation to remanufacture but is blocked completely by the supplier's aggressive strategy. The supplier's aggressive strategy costs it a fortune with a reduced profit, and the lower the $c_{r}$, the more aggressive the supplier's deterring strategy, and the lower the profit. Conversely, the manufacturer's possibility of getting into remanufacturing serves as an effective tool to strengthen its profit at the expense of the supplier, and the lower the $c_{r}$, the higher the manufacturer's profit. As a matter of fact, the aforesaid trend is extended to case $h-B$-2, where remanufacturing cannot be completely driven out of the market. Furthermore, as shown in Fig. 4 , the supplier in cases $h-B-1$ and $h-B-2$ achieves the highest profit under scenario $B$ owing to its more deterring tools at its disposal. Finally, if $c_{r}$ continues declining to case $h-C$, all used products are remanufactured. In this case, the supplier will price its components higher to enjoy the favourable market condition and the lower the $c_{r}$, the higher the wholesale price and the supplier's profit. On the other hand, the manufacturer will also benefit from a lower $c_{r}$ and the lower the $c_{r}$, the higher the manufacturer's profit. At the same $c_{r}$ in case $h$ - $C$, both supply chain members suffer lower profitability under scenario $N$ than other 
scenarios.

In addition, Fig. 4 clearly shows that, compared to the benchmark case $h$ - $A$, when $c_{r}$ enters into the range of cases $h$ - $B$-1 and $h-B-2$, the supplier's profitability suffers a continuous decline $\left(\left.\Pi_{S}^{h-A}\right|_{c_{r}=c_{r 4}^{h}}=\left.\Pi_{S}^{h-B-1}\right|_{c_{r}=c_{r 4}^{h}},\left.\Pi_{S}^{h-B-1}\right|_{c_{r}=c_{r 2}^{h}}=\left.\Pi_{S}^{h-B-2}\right|_{c_{r}=c_{r 2}^{h}}\right)$, but the manufacturer enjoys a continuous enhanced profitability as $c_{r}$ decreases $\left(\left.\Pi_{M}^{h-A}\right|_{c_{r}=c_{r 4}^{h}}=\left.\Pi_{M}^{h-B-1}\right|_{c_{r}=c_{r 4}^{h}},\left.\Pi_{M}^{h-B-1}\right|_{c_{r}=c_{r 2}^{h}}=\left.\Pi_{M}^{h-B-2}\right|_{c_{r}=c_{r 2}^{h}}\right)$. If $c_{r}$ continues decreasing into the range of case $h$ - $C$, the supplier's profit starts increasing when $c_{r}$ decreases $\left(\left.\Pi_{S}^{h-B-2}\right|_{c_{r}=c_{r 1}^{h}}=\left.\Pi_{S}^{h-C}\right|_{c_{r}=c_{r 1}^{h}}\right)$, but the manufacturer's profit suffers a sudden drop first $\left(\left.\Pi_{M}^{h-B-2}\right|_{c_{r}=c_{r 1}^{h}}>\left.\Pi_{M}^{h-C}\right|_{c_{r}=c_{r 1}^{h}}\right)$ before climbing up when $c_{r}$ decreases further. Therefore, when $c_{r}$ falls into case $h-C$ but is not efficient enough, both the supplier and the manufacturer are worse off in profitability compared to the benchmark case $h-A$. For low enough $c_{r}$, remanufacturing makes both members better off. From a managerial perspective, the manufacturer has the motivation to invest in remanufacturing technology so that $c_{r}$ is reduced to the ranges of $h-B-1$ and $h-B-2$ where its profitability is enhanced. Even if the manufacturer's profit will first drop when $c_{r}$ enters case $h-C$ from the right, it will eventually achieve a higher profitability than the benchmark case with further investment in remanufacturing technology to bring $c_{r}$ down to the lower end of $h$ - $C$. As a matter of fact, the supplier will also invest in changing component-design strategy in this case to enjoy a better profit. At a sufficiently low level of $c_{r}$ in case $h$ - $C$, remanufacturing benefits both members in profitability, resulting in a win-win scenario for both CLSC members.

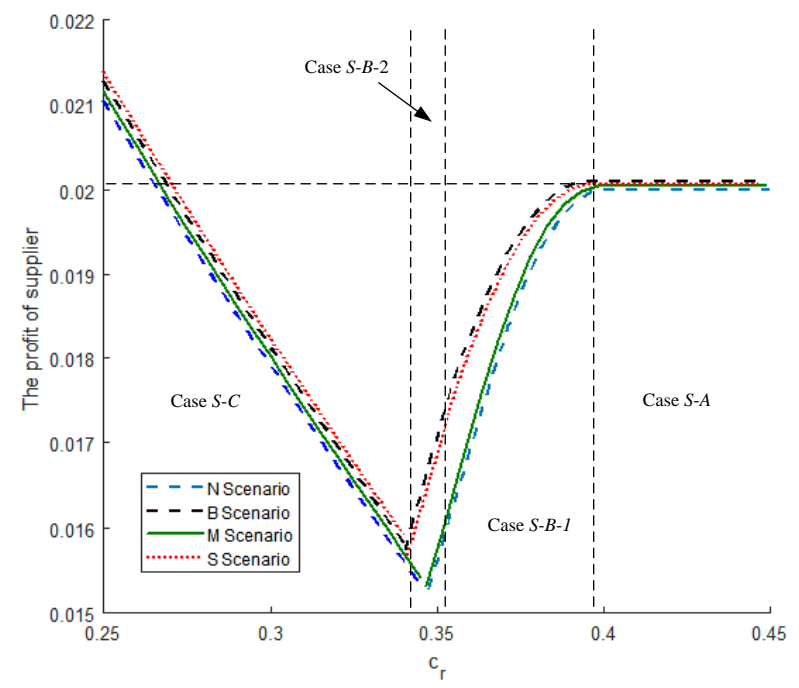

(a) The profit of the supplier

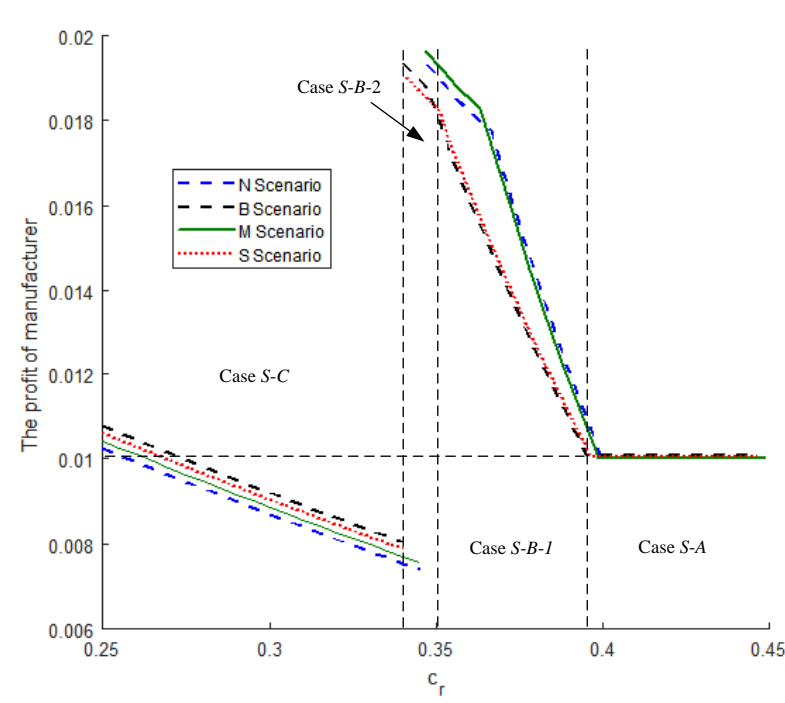

(b) The profit of the manufacturer

Fig 4. The effect of $c_{r}$ on the profits of the supplier and the manufacturer under four scenarios.

\subsection{Environmental impact analysis}

Based on the assumption on environmental impact of new and remanufactured products in Section 3 and equilibrium production quantities in Section 4, Result 5 can be obtained by analyzing the total environmental impact as a function of the base unit remanufacturing cost $c_{r}$ under scenario $h \in\{N, M, S\}$.

Result 5. (1) The total environmental impact of both new and remanufactured products is independent of $c_{r}$ in case $h$ - $A$, and decreases in $c_{r}$ in cases $h-B-1$ and $h$ - $C$. (2) In case $h-B-2$, the total environmental impact increases in $c_{r}$ if $\frac{e_{n}}{e_{r}}>\frac{2-\delta}{\delta}$.

Proof. See Appendix L. 
By using the same model parameter values in Fig. 3, one can draw the total environmental impact as a function of $c_{r}$ for the four scenarios in Fig. 5. Once again, the vertical dashed lines are the boundaries of different cases for scenario $S$ and identical to those in Fig. 3 and 4.

Based on Result 2 and the calculation of the total environmental impact, it is understandable that the total environmental impact of both new and remanufactured products is independent of $c_{r}$ in case $h-A$, and decreases in $c_{r}$ in cases $h-B$-1 and $h$ - $C$ for scenarios $N, M$, and $S$. Fig. 5 further confirms that the aforesaid relationships remain true for the general scenario $B$. However, in case $h-B-2$, both new and remanufactured products are present and substitutable in the market, a lower $c_{r}$ enhances demand for remanufactured products but depresses demand for new products. In this situation, the change of the total environmental impact depends on $e_{n} / e_{r}$. Given that $e_{n}$ and $e_{r}$ are the unit environmental impact of the new and remanufactured product, respectively, $e_{n} / e_{r}$ can be employed to represent the relative environmental advantage of the remanufactured product. The larger the $e_{n} / e_{r}$, the higher the environmental advantage of the remanufactured product. When $e_{n} / e_{r}$ is high enough (i.e. $\frac{e_{n}}{e_{r}}>\frac{2-\delta}{\delta}$ ), a smaller $c_{r}$ will result in a lower total environmental impact (see Fig. 5(b)). On the other hand, if $e_{n} / e_{r}$ is not sufficiently high (i.e. $\left.1<\frac{e_{n}}{e_{r}}<\min \left\{\frac{2 \beta_{k}(2-\delta)-\eta_{k}\left(\eta_{k}+\Delta_{k}\right)}{2 \beta_{k} \delta}, \frac{2\left(2 \beta_{o}-\eta_{o}^{2}\right)}{2 \beta_{o} \delta+\eta_{o} \Delta_{o}}-\frac{2 \beta_{o} \delta+\eta_{o} \Delta_{o}}{2 \beta_{o} \delta-\Delta_{o}^{2}}\right\}\right)$, a more efficient $c_{r}$ will actually cause an increase in the total environmental impact (see Fig. 5(a)).

By taking the environmental impact in case $h-A$ as a benchmark, we can further confirm that the total environmental impact is lower than that in the benchmark case for scenario $h$ if $\max \left\{0, c_{r 5}^{h}\right\}<c_{r}<\operatorname{com}\left\{c_{r 2}^{h}, c_{r 3}^{h}\right\}$; otherwise, the total environmental impact is higher than that in the benchmark case for scenario $h$, where $c_{r 5}^{N}=\delta-\frac{\left(1-c_{k}-c_{o}\right)}{e_{n}+e_{r}}\left(3 \delta e_{n}-e_{r}\right)$, $c_{r 5}^{S}=\delta-\frac{\left(1-c_{k}-c_{o}\right)}{e_{n}+e_{r}}\left(\frac{12 \beta_{k} \delta+\left(2 \eta_{k}-\Delta_{k}\right) \Delta_{k}}{4 \beta_{k}-\eta_{k}^{2}} e_{n}-e_{r}\right)$, and $c_{r 5}^{M}=\delta-\frac{\left(1-c_{k}-c_{o}\right)}{e_{n}+e_{r}}\left(\frac{6 \beta_{o} \delta+\left(2 \eta_{o}-\Delta_{o}\right) \Delta_{o}}{2 \beta_{o}-\eta_{o}^{2}} e_{n}-e_{r}\right)$. This result clearly indicates that a more favorable environmental impact can only occur in case $h$ - $C$. Fig. 5 shows this result graphically. Due to the limited display space, both Fig. 5(a) and 5(b) are truncated at $c_{r}=0.25$. If the declining lines in case $h$ - $C$ are extended further to the left (smaller $c_{r}$ ), it is possible that the total environmental impact may exceed the benchmark case. Therefore, remanufacturing activity is not always beneficial to the environment regardless of the supplier's and the manufacturer's product-design strategies.

A vertical comparison of the total environmental impact between scenarios $M$ and $N$ as well as between scenarios $S$ and $N$ leads to Result 6 as follows.

Result 6. The total environmental impact under scenarios $M$ and $S$ is higher (lower) than that under scenario $N$ in cases $h$ - $A$ and $h-C(h-B-1)$. It is also higher than that under scenario $N$ in case $h-B-2$ if $e_{n} / e_{r}>\max \left\{\bar{E}_{1}, \bar{E}_{2}\right\}$.

Proof. see Appendix M.

Based on Result 3 and the calculation of the total environmental impact, it is understandable that the total environmental impact under scenarios $M$ and $S$ is higher (lower) than that under scenario $N$ in cases $h-A$ and $h-C(h-B-1)$. The situation in case $h-B-2$ is more complicated: adjusting the product-design strategy enhances demand for new products but reduces demand for remanufactured products. If $e_{n} / e_{r}>\max \left\{\bar{E}_{1}, \bar{E}_{2}\right\}$, each unit of new product presumably generates significantly larger environmental impact than each unit of remanufactured product does. As such, the decreased environmental impact from 
reduced demand for remanufactured products cannot cover the increased environmental impact due to enhanced demand for new products, leading to a higher total environmental impact under scenarios $M$ and $S$ than that under scenario $N$. On the other hand, if $1<e_{n} / e_{r}<\min \left\{\bar{E}_{1}, \bar{E}_{2}\right\}$, the decreased environmental impact from reduced demand for remanufactured products surpasses the increased environmental impact from heightened demand for new products, resulting in a lower total environmental impact under scenarios $M$ and $S$ than that under scenario $N$. By following the aforesaid argument, we can infer that the total environmental impact under the general scenario $B$ is higher (lower) than that under scenario $N$ in case $h$ - $A$ and $h-C(h-B-1)$, and it is also higher than that under scenario $N$ in case $h-B-2$ if $e_{n} / e_{r}$ is sufficiently large. Fig. 5 visually illustrates this result.

Results 5 and 6 indicate that, if the manufacturer has motivation but is effectively blocked from remanufacturing, both members should be encouraged to adjust their product-design strategies to reduce the environmental impact. If remanufactured products are substitutes for new products, the two members should only be encouraged to adjust their product-design strategies if $e_{n} / e_{r}$ is not too high. When remanufacturing has overwhelming cost advantage, the manufacturer should be encouraged to remanufacture all used product because it produces a lower environmental impact than the benchmark case (no remanufacturing). But this encouraging policy has a limit: if remanufacturing is too efficient (i.e. $c_{r}<c_{r 5}^{h}$ ), dumping of remanufactured products may cause harm to environment. In addition, as the total environmental impact under scenario $N$ is the lowest compared to the other three scenarios (see Fig. 5), it is environmentally unwise to encourage the supplier or the manufacturer to change product design in this case. As we can see from Fig. 4 and Fig. 5, there exists a common range of $c_{r}$ in case $h-C$ such that remanufacturing achieves a triple-win result where both members' profitability will be enhanced and the total environmental impact will be reduced compared to the base case $h$ - $A$. Committing to product design by the members in this case further strengthens their profitability at the expense of environment, but this will not materially change the triple-win situation. This result has significant policy implications: From a policy-maker's perspective, the regulator can provide a subsidy to reduce $c_{r}$ if it is too high or impose an environmental fee to increase $c_{r}$ if it is too low so that $c_{r}$ falls within the appropriate triple-win range that benefits both environment and the two members.

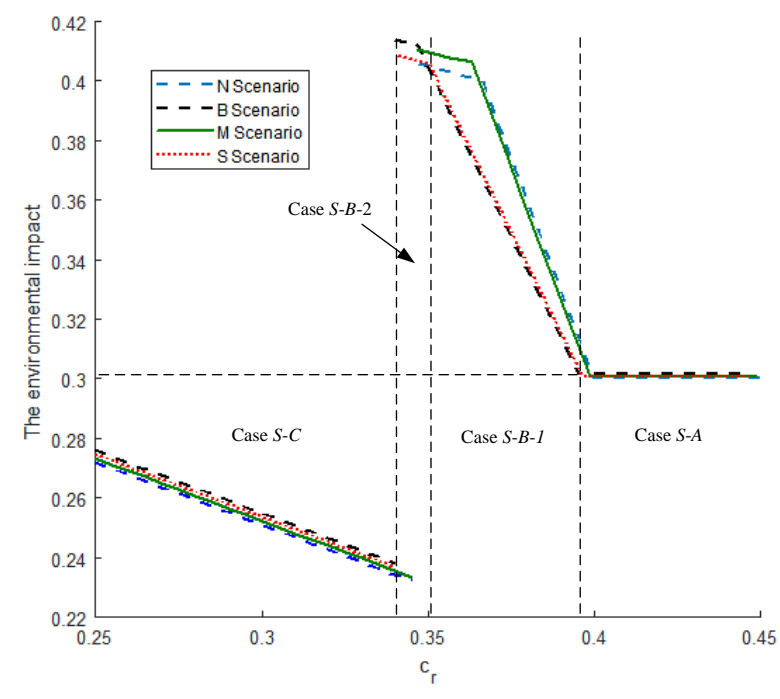

(a) $e_{n}=3$ and $e_{r}=1.2$

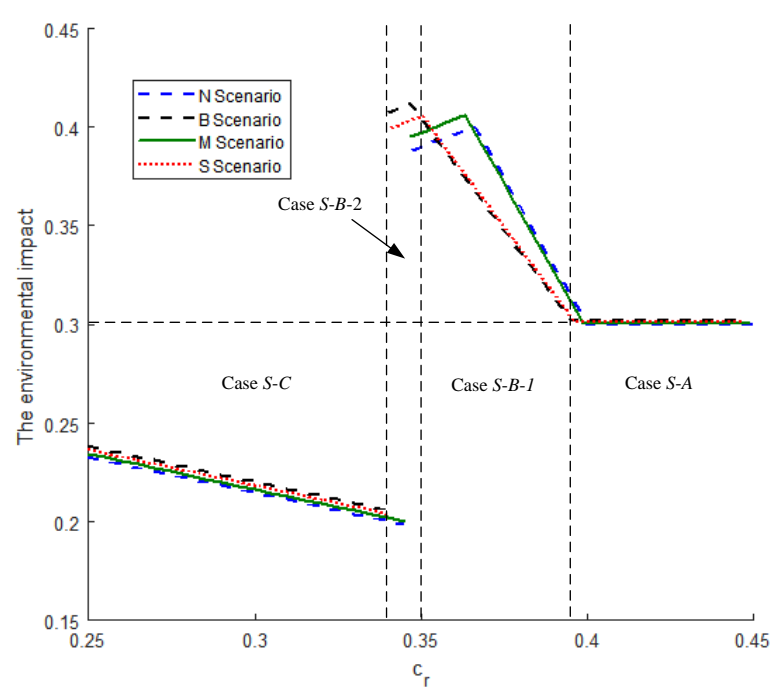

(b) $e_{n}=3$ and $e_{r}=0.6$

Fig 5. The total environmental impact under four scenarios 


\section{Conclusions}

Product design has an important impact on remanufacturing of used products and it interacts with other pricing and/or production quantity decisions. Although current literature has already explored this issue, most studies assume that products are designed and produced by an integrated manufacturer. This assumption is inconsistent with industrial practices as it ignores the important role of suppliers in product design, production and remanufacturing. In this paper, we model a two-echelon CLSC consisting of a key component supplier and a non-integrated manufacturer. The supplier controls the remanufacturability level of the key component and the non-integrated manufacturer determines the remanufacturability level of the final product (excluding the key component), purchases key components from the supplier to produce new products, and remanufactures used products. Thus, their relationships can be both competitive and cooperative. The general model formulates their interaction in a single-stage analytic framework where the supplier is the leader, followed by the manufacturer, and both members adjust their product-design strategies. The base unit remanufacturing cost is adopted as the key parameter to characterize the equilibrium solution structure and examine the manufacturer's remanufacturing strategy. We then explore how different model parameters affect product-design and production quantity decisions. Subsequently, we assess how the base unit remanufacturing cost and different product-design strategies influence the profitability of the two members and environmental performance of the CLSC. Key findings are as follows.

In the supplier-dominated CLSC, the manufacturer's decision to commit to no, partial, and complete remanufacturing is contingent upon the remanufacturing cost advantage and consumer's willingness-to-pay for remanufactured products. If consumer's willingness-to-pay is sufficiently high for remanufactured products, the manufacturer may skip partial remanufacturing and jump from no to complete remanufacturing directly as the base unit remanufacturing cost decreases. When remanufacturing has moderate cost advantage, the supplier and manufacturer can employ negative product design on top of the supplier's aggressive component pricing strategy to block remanufacturing more effectively.

The supplier's component-design strategy has a significant impact on the manufacturer's product-design strategy and production quantity of new and remanufactured products. When the manufacturer does not remanufacture, it takes a negative product-design strategy, and this strategy and the quantity of new products are independent of the component remanufacturability level. When the manufacturer commits to partial remanufacturing, as the supplier's component remanufacturability level increases, the manufacturer's product remanufacturability level and the quantity of remanufactured products increase, but the quantity of new products decreases. When the manufacturer remanufactures all used products, as the supplier's component remanufacturability level increases, the quantity of new and remanufactured products increases, but the manufacturer's product remanufacturability decision is contingent upon the impact of this decision on the unit base production cost of remanufactured and new products.

Remanufacturing is not always beneficial to the CLSC members or the environment, but adjusting product-design strategies can reduce the negative impact under certain conditions compared to the scenario when none of them changes its product-design strategy. When the manufacturer has motivation to remanufacture but is completely blocked or commits to partial remanufacturing, the manufacturer benefits, but the supplier suffers and this loss can be curbed by adjusting their product-design strategies. These two cases see aggravated environmental burdens but adjusting product-design strategies may 
mitigate the negative environmental impact. On the other hand, when remanufacturing has overwhelming cost advantage and the manufacturer remanufactures all used products, our analysis indicates that there exists an appropriate range of the base unit remanufacturing cost such that both members enjoy higher profitability at a lower total environmental impact. In this case, implementing product design will further boost profitability at the expense of environment, but the triple-win result in both members' profitability and environmental performance will not be sacrificed. Policy implications are that the regulator can foster the triple-win result by imposing an environmental fee or furnishing a subsidy.

\section{References}

Agrawal, V. V., Atasu, A., Van Ittersum, K., 2015. Remanufacturing, third-party competition, and consumers' perceived value of new products. Management Science 61, 60-72.

Agrawal, V. V., Ferguson, M., Toktay, L. B., Thomas, V. M., 2012. Is leasing greener than selling? Management Science 58, 523-533.

Aras, N., Verter, V., Boyaci, T., 2006. Coordination and priority decisions in hybrid manufacturing/remanufacturing systems. Production and Operations Management 15, 528-543.

Atasu, A., Guide Jr, V. D. R., Van Wassenhove, L. N., 2010. So what if remanufacturing cannibalizes my new product sales? California Management Review 52, 56-76.

Atasu, A., Sarvary, M., Van Wassenhove, L. N., 2008. Remanufacturing as a marketing strategy. Management Science 54(10), 1731-1746.

Atasu, A., Souza, G. C., 2013. How does product recovery affect quality choice? Production and Operations Management 22, 991-1010.

Badri, H., Ghomi, S. M. T. F., Hejazi, T. H., 2017. A two-stage stochastic programming approach for value-based closed-loop supply chain network design. Transportation Research Part E: Logistics and Transportation Review 105, 1-17.

Biswas, I., Raj, A., Srivastava, S. K., 2018. Supply chain channel coordination with triple bottom line approach. Transportation Research Part E: Logistics and Transportation Review 115, 213-226.

Cachon, G. P., Lariviere, M. A., 1999. Capacity allocation using past sales: When to turn-and-earn. Management Science 45, 685-703.

Choi, T. M., Li, Y., Xu, L., 2013. Channel leadership, performance and coordination in closed loop supply chains. International Journal of Production Economics 146(1), 371-380.

Choi, T. M., 2017. Pricing and branding for remanufactured fashion products. Journal of Cleaner Production 165, $1385-1394$.

Chan, K. C., Zhou, Y., Wong, K. H., 2018. A dynamic equilibrium model of the oligopolistic closed-loop supply chain network under uncertain and time-dependent demands. Transportation Research Part E: Logistics and Transportation Review 118, 325-354.

Debo, L. G., Toktay, L. B., Van Wassenhove, L. N., 2005. Market segmentation and product technology selection for remanufacturable products. Management Science 51, 1193-1205.

DeCroix, G. A., 2006. Optimal policy for a multiechelon inventory system with remanufacturing. Operations Research 54, 532-543.

Desai, P., Kekre, S., Radhakrishnan, S., Srinivasan, K., 2001. Product differentiation and commonality in design: Balancing revenue and cost drivers. Management Science 47, 37-51.

Esenduran, G., Kemahlığlu-Ziya, E., Swaminathan, J. M., 2017. Impact of take-back regulation on the remanufacturing 
Industry. Production and Operations Management 26, 924-944.

Ferguson, M. E., Toktay, L. B., 2006. The effect of competition on recovery strategies. Production and Operations Management 15, 351-368.

Ferrer, G., Swaminathan, J. M., 2010. Managing new and differentiated remanufactured products. European Journal of Operational Research 203, 370-379.

Fisher, M. L., Ittner, C. D., 1999. The impact of product variety on automobile assembly operations: empirical evidence and simulation analysis. Management Science 45(6), 771-786.

Galbreth, M. R., Boyac1, T., Verter, V., 2013. Product reuse in innovative industries. Production and Operations Management 22, 1011-1033.

Gaur, J., Amini, M., Rao, A. K., 2017. Closed-loop supply chain configuration for new and reconditioned products: An integrated optimization model. Omega 66, 212-223.

Giutini, R., Gaudette, K., 2003. Remanufacturing: The next great opportunity for boosting US productivity. Business Horizons 46, 41-48.

Goyal, M., Netessine, S., 2007. Strategic technology choice and capacity investment under demand uncertainty. Management Science 53, 192-207.

Gu, W., Chhajed, D., Petruzzi, N. C., Yalabik, B., 2015. Quality design and environmental implications of green consumerism in remanufacturing. International Journal Production Economics 162, 55-69.

Guide Jr, V. D. R., Li, J., 2010. The potential for cannibalization of new products sales by remanufactured products. Decision Sciences 41, 547-572.

Hatcher, G. D., Ijomah, W. L., Windmill, J. F. C., 2011. Design for remanufacture: a literature review and future research needs. Journal of Cleaner Production 19, 2004-2014.

Hong, Z. F., Wang, H., Gong, Y. M., 2019. Green product design considering functional-product reference. International Journal of Production Economics. http://doi.org/10.1016/j.ijpe.2019.01.008.

Huang, Y., Wang, Z., 2017. Values of information sharing: A comparison of supplier-remanufacturing and manufacturer-remanufacturing scenarios. Transportation Research Part E: Logistics and Transportation Review 106, $20-44$.

Jacobs, B. W., Subramanian, R., 2012. Sharing responsibility for product recovery across the supply chain. Production and Operations Management 21, 85-100.

Johnson, J. P., Myatt, D. P., 2006. Multiproduct cournot oligopoly. The RAND Journal of Economics 37, 583-601.

Joshi, A. D., Gupta, S. M., 2019. Evaluation of design alternatives of End-Of-Life products using internet of things. International Journal of Production Economics 208, 281-293.

Kim, K., Chhajed, D., 2000. Commonality in product-design: Cost saving, valuation change and cannibalization. European Journal of Operational Research 125, 602-621.

Li, W., Chen, J., 2018. Backward integration strategy in a retailer Stackelberg supply chain. Omega 75, 118-130.

Liu, W. J., Wu, C. F., Chang, X. Y., Chen, Y., Liu, S. F., 2017. Evaluating remanufacturing industry of China using an improved grey fixed weight clustering method-a case of Jiangsu province. Journal of Cleaner Production 142, 2006-2020.

Liu, Z., Tang, J., Li, B.Y., Wang, Z., 2017. Trade-off between remanufacturing and recycling of WEEE and the environmental implication under the Chinese Fund Policy. Journal of Cleaner Production 167, 97-109.

Ma, P., Li, K. W., Wang, Z. J., 2017. Pricing decisions in closed-loop supply chains with marketing effort and fairness concerns. International Journal of Production Research 55, 1-22.

Majumder, P., Groenevelt, H., 2001. Competition in remanufacturing. Production and Operations Management 10, $125-141$.

Ma, Z. J., Zhou, Q., Dai, Y., Sheu, J. B., 2017. Optimal pricing decisions under the coexistence of "trade old for new" and 
"trade old for remanufactured programs". Transportation Research Part E: Logistics and Transportation Review 106: $337-352$.

McConocha, D. M., Speh, T. W., 1991. Remarketing: Commercialization of remanufacturing technology. Journal of Business and Industrial Marketing 6, 23-37.

Mukhopadhyay, S. K., Setoputro, R., 2005. Optimal return policy and modular design for build-to-order products. Journal of Operations Management 23, 496-506.

Oraiopoulos, N., Ferguson, M. E., Toktay, L. B., 2012. Relicensing as a secondary market strategy. Management Science 58(5), $1022-1037$.

Örsdemir, A., Kemahlıoğlu-Ziya, E., Parlaktürk, A. K., 2014. Competitive quality choice and remanufacturing. Production and Operations Management 23, 48-64.

Pangburn, M. S., Stavrulaki, E., 2014. Take back costs and product durability. European Journal of Operational Research 238, 175-184.

Qiang, Q. P., 2015. The closed-loop supply chain network with competition and design for remanufactureability. Journal of Cleaner Production 105, 348-356.

Raz, G., Ovchinnikov, A., Blass, V., 2017. Economic, environmental, and social impact of remanufacturing in a competitive setting. IEEE Transactions on Engineering Management 64, 1-15.

Reimann, M., Xiong, Y., Zhou, Y., 2019. Managing a closed-loop supply chain with process innovation for remanufacturing. European Journal of Operational Research. https://doi.org/10.1016/j.ejor.2019.01.028.

Savaskan, R. C., Bhattacharya, S., Van Wassenhove, L. N., 2004. Closed-loop supply chain models with product remanufacturing. Management Science 50, 239-252.

Savaskan, R. C., Van Wassenhove, L. N., 2006. Reverse channel design: the case of competing retailers. Management Science $52,1-14$.

Shi, T., Gu, W., Chhajed, D., Petruzzi, N. C., 2016. Effects of remanufacturable product design on market segmentation and the environment. Decision Sciences 47, 298-332.

Steeneck, D. W., Sarin, S. C., 2018. Product design for leased products under remanufacturing. International Journal of Production Economics 202,131-144.

Subramanian, R., Ferguson, M. E., Toktay, L. B, 2013. Remanufacturing and the component commonality decision. Production and Operations Management 22, 36-53.

Taleizadeh, A. A., Soleymanfar, V. R., Choi, T. M., 2017. Optimal pricing and alliance strategy in a retailer-led supply chain with the return policy: A game-theoretic analysis. Information Sciences 420, 466-489.

Wan, X., Sanders, N. R., 2017. The negative impact of product variety: Forecast bias, inventory levels, and the role of vertical integration. International Journal of Production Economics 186, 123-131.

Wu, C. H., 2012. Product-design and pricing strategies with remanufacturing. European Journal of Operational Research 222, 204-215.

Wu, C. H., 2013. OEM product design in a price competition with remanufactured product. Omega 41, 287-298.

Xiong, Y., Zhao, Q., Zhou, Y., 2016. Manufacturer-remanufacturing vs supplier-remanufacturing in a closed-loop supply chain. International Journal Production Economics 176, 21-28.

Xiong, Y., Zhou, Y., Li, G., Chan, H. K., Xiong, Z., 2013. Don’t forget your supplier when remanufacturing. European Journal of Operational Research 230, 15-25.

Yan, T., Ribbink, D., Pun, H., 2018. Incentivizing supplier participation in buyer innovation: Experimental evidence of non-optimal contractual behaviors. Journal of Operations Management 57, 36-53.

Yenipazarli, A., 2016. Managing new and remanufactured products to mitigate environmental damage under emissions 
regulation. European Journal of Operational Research 249, 117-130.

Zheng, X. X., Liu, Z., Li, K. W., Huang, J., Chen, J., 2019. Cooperative game approaches to coordinating a three-echelon closed-loop supply chain with fairness concerns. International Journal of Production Economics. https://doi.org/10.1016/j.ijpe.2019.01.011.

\section{Appendices: Supplementary materials}

\section{Appendix A. Proof of Proposition 1}

The Lagrangian and the Karush-Kuhn-Tucker optimality conditions for the manufacturer's optimization problem are stated as follows:

$$
\begin{aligned}
& L_{M}^{B}\left(q_{n}, q_{r}, \gamma_{o}\right)=\left(1-q_{n}-\delta q_{r}-\omega-c_{o}-\eta_{o} \gamma_{o}\right) q_{n}+\left(\delta\left(1-q_{n}-q_{r}\right)-c_{r}+\gamma_{k} \Delta_{k}+\gamma_{o} \Delta_{o}\right) q_{r}-\frac{1}{2} \beta_{o} \gamma_{o}^{2}+\lambda_{1}\left(q_{n}-q_{r}\right)+\lambda_{2} q_{r} \\
& \partial L_{M}^{B} / \partial q_{n}=1-2 q_{n}-2 \delta q_{r}-\omega-c_{o}-\gamma_{o} \eta_{o}+\lambda_{1}=0 \\
& \partial L_{M}^{B} / \partial q_{r}=\delta-c_{r}-2 \delta q_{n}-2 \delta q_{r}+\gamma_{k} \Delta_{k}+\gamma_{o} \Delta_{o}-\lambda_{1}+\lambda_{2}=0 \\
& \partial L_{M}^{B} / \partial \gamma_{o}=q_{r} \Delta_{o}-\beta_{o} \gamma_{o}-q_{n} \eta_{o}=0 \\
& \lambda_{1}\left(q_{n}-q_{r}\right)=\lambda_{2} q_{r}=0 \\
& q_{n}-q_{r} \geq 0, q_{r} \geq 0
\end{aligned}
$$

where $\lambda_{1}$ and $\lambda_{2}$ are Lagrangian multipliers. According to the Karush-Kuhn-Tucker condition, there are four possible cases for the optimal solutions corresponding to different combinations of nonnegative Lagrangian coefficients $\lambda_{1}$ and $\lambda_{2}$ as follows.

Case I: When $\quad \lambda_{1}=0 \quad$ and $\quad \lambda_{2}>0$, the optimal solutions are $q_{n}^{B-A}=\frac{2 \delta \beta_{o}\left(1-c_{o}+c_{r}-\omega-\gamma_{k} \Delta_{k}-\delta\right)-\Delta_{o}\left(\eta_{o}\left(\delta+\gamma_{k} \Delta_{k}-c_{r}\right)+\Delta_{o}\left(1-c_{o}-\omega\right)\right)-\left(2 \delta \beta_{o}+\Delta_{o} \eta_{o}\right) \lambda_{2}}{2\left(2 \beta_{o} \delta(1-\delta)-\left(\delta \eta_{o}^{2}+2 \delta \eta_{o} \Delta_{o}+\Delta_{o}^{2}\right)\right)}$ $q_{r}^{B-A}=\frac{2 \beta_{o}\left(\delta\left(c_{o}+\omega\right)+\gamma_{k} \Delta_{k}-c_{r}\right)-\eta_{o}^{2}\left(\delta+\gamma_{k} \Delta_{k}-c_{r}\right)-\eta_{o} \Delta_{o}\left(1-c_{o}-\omega\right)+\lambda_{2}\left(2 \beta_{o}-\eta_{o}^{2}\right)}{2\left(2 \beta_{o} \delta(1-\delta)-\left(\delta \eta_{o}^{2}+2 \delta \eta_{o} \Delta_{o}+\Delta_{o}^{2}\right)\right)}$

$\gamma_{o}^{B-A}=\frac{\Delta_{o}\left(\delta\left(c_{o}+\omega\right)+\gamma_{k} \Delta_{k}-c_{r}\right)-\delta \eta_{o}\left(1-c_{o}+c_{r}-\omega-\gamma_{k} \Delta_{k}-\delta\right)-\lambda_{2}\left(\Delta_{o}+\delta \eta_{o}\right)}{2 \beta_{o} \delta(1-\delta)-\left(\delta \eta_{o}^{2}+2 \eta_{o} \delta \Delta_{o}+\Delta_{o}^{2}\right)}$. Then, $q_{n}^{B-A}-q_{r}^{B-A}>0$ and $q_{r}^{B-A}=0 \quad$ can be derived based on the Complementary Relaxation Theorem. It is easy to get $\lambda_{2}=c_{r}-\delta-\gamma_{k} \Delta_{k}+\frac{\left(1-c_{o}-w\right)\left(2 \beta_{o} \delta+\Delta_{o} \eta_{o}\right)}{2 \beta_{o}-\eta_{o}^{2}}$ given $q_{r}^{B-A}=0$. By substituting it into $q_{n}^{B-A}$ and $\gamma_{o}^{B-A}$, we have $q_{n}^{B-A}=\frac{\beta_{o}\left(1-c_{o}-\omega\right)}{2 \beta_{o}-\eta_{o}^{2}}$ and $\gamma_{o}^{B-A}=-\frac{\eta_{o}\left(1-c_{o}-\omega\right)}{2 \beta_{o}-\eta_{o}{ }^{2}}$. Furthermore, $c_{r}>\delta+\gamma_{k} \Delta_{k}-\frac{\left(1-c_{o}-\omega\right)\left(2 \beta_{o} \delta+\Delta_{o} \eta_{o}\right)}{2 \beta_{o}-\eta_{o}{ }^{2}}$ is derived from $\lambda_{2}>0$, corresponding to the condition for Case I and denoted by $B$ - $A$. This condition $c_{r}>\delta+\gamma_{k} \Delta_{k}-\frac{\left(1-c_{o}-\omega\right)\left(2 \beta_{o} \delta+\Delta_{o} \eta_{o}\right)}{2 \beta_{o}-\eta_{o}^{2}}$ can be equivalently expressed as $\omega<1-c_{o}-\frac{\left(2 \beta_{o}-\eta_{o}^{2}\right)\left(\delta+\gamma_{k} \Delta_{k}-c_{r}\right)}{2 \beta_{o} \delta+\eta_{o} \Delta_{o}}$

Case II: When $\lambda_{1}=0$ and $\lambda_{2}=0$, the optimal solutions are $q_{n}^{B-B}=\frac{2 \delta \beta_{o}\left(1-c_{o}+c_{r}-\omega-\gamma_{k} \Delta_{k}-\delta\right)-\Delta_{o}\left(\eta_{o}\left(\delta+\gamma_{k} \Delta_{k}-c_{r}\right)+\Delta_{o}\left(1-c_{o}-\omega\right)\right)}{2\left(2 \beta_{o} \delta(1-\delta)-\left(\delta \eta_{o}^{2}+2 \delta \eta_{o} \Delta_{o}+\Delta_{o}^{2}\right)\right)}$, $q_{r}^{B-B}=\frac{2 \beta_{o}\left(\delta\left(c_{o}+\omega\right)+\gamma_{k} \Delta_{k}-c_{r}\right)-\eta_{o}^{2}\left(\delta+\gamma_{k} \Delta_{k}-c_{r}\right)-\eta_{o} \Delta_{o}\left(1-c_{o}-\omega\right)}{2\left(2 \beta_{o} \delta(1-\delta)-\left(\delta \eta_{o}^{2}+2 \delta \eta_{o} \Delta_{o}+\Delta_{o}^{2}\right)\right)}$, and $\gamma_{o}^{B-B}=\frac{\Delta_{o}\left(\delta\left(c_{o}+\omega\right)+\gamma_{k} \Delta_{k}-c_{r}\right)-\delta \eta_{o}\left(1-c_{o}+c_{r}-\omega-\gamma_{k} \Delta_{k}-\delta\right)}{2 \beta_{o} \delta(1-\delta)-\left(\delta \eta_{o}^{2}+2 \eta_{o} \delta \Delta_{o}+\Delta_{o}^{2}\right)}$. Then, $q_{n}^{B-B}-q_{r}^{B-B} \geq 0$ and $q_{r}^{B-B} \geq 0$ can be obtained from the Complementary Relaxation Theorem. Therefore, we obtain 
the condition for Case II as $\delta+\gamma_{k} \Delta_{k}-\frac{\left(1-c_{o}-\omega\right)\left(4 \beta_{o} \delta-\Delta_{o}\left(\Delta_{o}-\eta_{o}\right)\right)}{2 \beta_{o}(1+\delta)+\left(\Delta_{o}-\eta_{o}\right) \eta_{o}} \leq c_{r} \leq \delta+\gamma_{k} \Delta_{k}-\frac{\left(1-c_{o}-\omega\right)\left(2 \beta_{o} \delta+\Delta_{o} \eta_{o}\right)}{2 \beta_{o}-\eta_{o}{ }^{2}}$, denoted by $B$ - $B$, which can be rewritten as $1-c_{o}-\frac{\left(2 \beta_{o}-\eta_{o}^{2}\right)\left(\delta+\gamma_{k} \Delta_{k}-c_{r}\right)}{2 \beta_{o} \delta+\eta_{o} \Delta_{o}} \leq \omega \leq 1-c_{o}-\frac{\left(2 \beta_{o}(1+\delta)+\eta_{o}\left(\Delta_{o}-\eta_{o}\right)\right)\left(\delta+\gamma_{k} \Delta_{k}-c_{r}\right)}{4 \beta_{o} \delta-\Delta_{o}\left(\Delta_{o}-\eta_{o}\right)}$.

Case III: If $\lambda_{1}>0$ and $\lambda_{2}=0$, the optimal solutions are $q_{n}^{B-C}=\frac{2 \delta \beta_{o}\left(1-c_{o}+c_{r}-\omega-\gamma_{k} \Delta_{k}-\delta\right)-\Delta_{o}\left(\eta_{o}\left(\delta+\gamma_{k} \Delta_{k}-c_{r}\right)+\Delta_{o}\left(1-c_{o}-\omega\right)\right)+\left(4 \beta_{o} \delta-\Delta_{o}\left(\Delta_{o}-\eta_{o}\right)\right) \lambda_{1}}{2\left(2 \beta_{o} \delta(1-\delta)-\left(\delta \eta_{o}^{2}+2 \delta \eta_{o} \Delta_{o}+\Delta_{o}^{2}\right)\right)}$

$q_{r}^{B-C}=\frac{2 \beta_{o}\left(\delta\left(c_{o}+\omega\right)+\gamma_{k} \Delta_{k}-c_{r}\right)-\eta_{o}^{2}\left(\delta+\gamma_{k} \Delta_{k}-c_{r}\right)-\eta_{o} \Delta_{o}\left(1-c_{o}-\omega\right)-\left(2 \beta_{o}(1+\delta)+\left(\Delta_{o}-\eta_{o}\right) \eta_{o}\right) \lambda_{1}}{2\left(2 \beta_{o} \delta(1-\delta)-\left(\delta \eta_{o}^{2}+2 \delta \eta_{o} \Delta_{o}+\Delta_{o}^{2}\right)\right)}$

$\gamma_{o}^{B-C}=\frac{\Delta_{o}\left(\delta\left(c_{o}+\omega\right)+\gamma_{k} \Delta_{k}-c_{r}\right)-\delta \eta_{o}\left(1-c_{o}+c_{r}-\omega-\gamma_{k} \Delta_{k}-\delta\right)-\lambda_{1}\left(\Delta_{o}+\delta \Delta_{o}+2 \delta \eta_{o}\right)}{2 \beta_{o} \delta(1-\delta)-\left(\delta \eta_{o}{ }^{2}+2 \delta \eta_{o} \Delta_{o}+\Delta_{o}^{2}\right)}$. Then, $\quad q_{n}^{B-C}-q_{r}^{B-C}=0 \quad$ and $\quad q_{r}^{B-C}>0 \quad$ can $\quad$ be confirmed by the Complementary Relaxation Theorem. Solving equation $q_{n}^{B-C}-q_{r}^{B-C}=0$ yields $\lambda_{1}=\frac{2 \beta_{o}\left(\gamma_{k} \Delta_{k}(1+\delta)-\delta\left(1-2 c_{o}-2 \omega-\delta\right)\right)+\left(\Delta_{o}-\eta_{o}\right)\left(\left(1-\omega-c_{o}\right) \Delta_{o}+\left(\delta+\gamma_{k} \Delta_{k}\right) \eta_{o}\right)-c_{r}\left(2 \beta_{o}(1+\delta)+\eta_{o}\left(\Delta_{o}-\eta_{o}\right)\right)}{2 \beta_{o}(1+3 \delta)-\left(\Delta_{o}-\eta_{o}\right)^{2}}$. Substituting it into $q_{n}^{B-C}, q_{r}^{B-C}$ and $\gamma_{o}^{B-C}$ results in $q_{n}^{B-C}=q_{r}^{B-C}=\frac{\beta_{o}\left(1-c_{r}-\omega-c_{o}+\delta+\gamma_{k} \Delta_{k}\right)}{2 \beta_{o}(1+3 \delta)-\left(\Delta_{o}-\eta_{o}\right)^{2}}$ and $\gamma_{o}^{B-C}=\frac{\left(1-c_{r}-\omega-c_{o}+\delta+\gamma_{k} \Delta_{k}\right)\left(\Delta_{o}-\eta_{o}\right)}{2 \beta_{o}(1+3 \delta)-\left(\Delta_{o}-\eta_{o}\right)^{2}}$. As $\lambda_{1}>0$ and $q_{r}^{B-C}>0$, the condition for Case III is $c_{r}<\delta+\gamma_{k} \Delta_{k}-\frac{\left(1-c_{o}-\omega\right)\left(4 \beta_{o} \delta-\Delta_{o}\left(\Delta_{o}-\eta_{o}\right)\right)}{2 \beta_{o}(1+\delta)+\left(\Delta_{o}-\eta_{o}\right) \eta_{o}}$, denoted by $B$-C, which can be rearranged as $\omega>1-c_{o}-\frac{\left(2 \beta_{o}(1+\delta)+\eta_{o}\left(\Delta_{o}-\eta_{o}\right)\right)\left(\delta+\gamma_{k} \Delta_{k}-c_{r}\right)}{4 \beta_{o} \delta-\Delta_{o}\left(\Delta_{o}-\eta_{o}\right)}$.

Case IV: If $\lambda_{1}>0$ and $\lambda_{2}>0, q_{n}^{B-D}=q_{r}^{B-D}=0$ can be verified by the Complementary Relaxation Theorem, corresponding to a case without any production activity, which should be neglected.

Proposition 1 is thus proved.

\section{Appendix B. Proof of Corollary 1}

By examining the relevant first order partial derivatives, the sensitivity analyses of the manufacturer's decisions of the optimal quantity of new and remanufactured product decisions with respect to $\omega$ and $\gamma_{k}$ are presented as follows:

(1) $\quad \frac{\partial q_{n}^{B-A}}{\partial \omega}=-\frac{\beta_{o}}{2 \beta_{o}-\eta_{o}{ }^{2}}<0 \quad, \quad \frac{\partial q_{n}^{B-A}}{\partial \gamma_{k}}=0 \quad, \quad \frac{\partial q_{n}^{B-B}}{\partial \omega}=-\frac{2 \beta_{o} \delta-\Delta_{o}^{2}}{2\left(2 \beta_{o} \delta(1-\delta)-\left(\delta \eta_{o}{ }^{2}+2 \delta \eta_{o} \Delta_{o}+\Delta_{o}^{2}\right)\right)}<0$

$\frac{\partial q_{n}^{B-B}}{\partial \gamma_{k}}=-\frac{\Delta_{k}\left(2 \beta_{o} \delta+\Delta_{o} \eta_{o}\right)}{2\left(2 \beta_{o} \delta(1-\delta)-\left(\delta \eta_{o}^{2}+2 \delta \eta_{o}\right.\right.}$
$\frac{\partial q_{n}^{B-C}}{\partial \gamma_{k}}=\frac{\beta_{o} \Delta_{k}}{2 \beta_{o}(1+3 \delta)-\left(\Delta_{o}-\eta_{o}\right)^{2}}>0$.

(2) $\frac{\partial q_{r}^{B-A}}{\partial \omega}=0, \frac{\partial q_{r}^{B-A}}{\partial \gamma_{k}}=0, \frac{\partial q_{r}^{B-B}}{\partial \omega}=\frac{2 \beta_{o} \delta+\Delta_{o} \eta_{o}}{2\left(2 \beta_{o} \delta(1-\delta)-\left(\delta \eta_{o}{ }^{2}+2 \delta \eta_{o} \Delta_{o}+\Delta_{o}^{2}\right)\right)}>0, \frac{\partial q_{r}^{B-B}}{\partial \gamma_{k}}=\frac{\Delta_{k}\left(2 \beta_{o}-\eta_{o}{ }^{2}\right)}{2\left(2 \beta_{o} \delta(1-\delta)-\left(\delta \eta_{o}{ }^{2}+2 \delta \eta_{o} \Delta_{o}+\Delta_{o}^{2}\right)\right)}>0$, $\frac{\partial q_{r}^{B-C}}{\partial \omega}=-\frac{\beta_{o}}{2 \beta_{o}(1+3 \delta)-\left(\Delta_{o}-\eta_{o}\right)^{2}}<0$, and $\frac{\partial q_{r}^{B-C}}{\partial \gamma_{k}}=\frac{\beta_{o} \Delta_{k}}{2 \beta_{o}(1+3 \delta)-\left(\Delta_{o}-\eta_{o}\right)^{2}}>0$.

Corollary 1 is thus confirmed.

\section{Appendix C. Proof of Corollary 2}


By examining the relevant first order partial derivatives, the sensitivity analyses of the manufacturer's decision of the optimal product remanufacturability level with respect to the $\omega$ and $\gamma_{k}$ are presented as follows:

$$
\begin{aligned}
& \frac{\partial \gamma_{o}^{B-A}}{\partial \omega}=\frac{\eta_{o}}{2 \beta_{o}-\eta_{o}{ }^{2}}>0 \quad, \quad \frac{\partial \gamma_{o}^{B-A}}{\partial \gamma_{k}}=0 \quad, \quad \frac{\partial \gamma_{o}^{B-B}}{\partial \omega}=\frac{\delta\left(\Delta_{o}+\eta_{o}\right)}{2 \beta_{o} \delta(1-\delta)-\left(\delta \eta_{o}{ }^{2}+2 \delta \eta_{o} \Delta_{o}+\Delta_{o}^{2}\right)}>0 \\
& \frac{\partial \gamma_{o}^{B-B}}{\partial \gamma_{k}}=\frac{\Delta_{k}\left(\Delta_{o}+\delta \eta_{o}\right)}{2 \beta_{o} \delta(1-\delta)-\left(\delta \eta_{o}^{2}+2 \delta \eta_{o} \Delta_{o}+\Delta_{o}^{2}\right)}>0, \frac{\partial \gamma_{o}^{B-C}}{\partial \omega}=-\frac{\Delta_{o}-\eta_{o}}{2 \beta_{o}(1+3 \delta)-\left(\Delta_{o}-\eta_{o}\right)^{2}}, \text { and } \frac{\partial \gamma_{o}^{B-C}}{\partial \gamma_{k}}=\frac{\Delta_{k}\left(\Delta_{o}-\eta_{o}\right)}{2 \beta_{o}(1+3 \delta)-\left(\Delta_{o}-\eta_{o}\right)^{2}} .
\end{aligned}
$$

Given that $2 \beta_{o}(1+3 \delta)-\left(\Delta_{o}-\eta_{o}\right)^{2}>0$ is equivalent to the condition that guarantees the existence of optimal solutions in Proposition 1, one can derive that $\frac{\partial \gamma_{o}^{B-C}}{\partial \omega}<0$ and $\frac{\partial \gamma_{o}^{B-C}}{\partial \gamma_{k}}>0$ if $\Delta_{o}-\eta_{o}>0$; Otherwise, $\frac{\partial \gamma_{o}^{B-C}}{\partial \omega}>0$ and $\frac{\partial \gamma_{o}^{B-C}}{\partial \gamma_{k}}<0$.

Corollary 2 is thus confirmed.

\section{Appendix D. Proof of equilibrium solution under scenario $N$}

The Lagrangian and the Karush-Kuhn-Tucker optimality conditions for the manufacturer's optimization problem are stated as follows:

$$
\begin{aligned}
& L_{M}^{N}\left(q_{n}, q_{r}\right)=\left(1-q_{n}-\delta q_{r}-\omega-c_{o}\right) q_{n}+\left(\delta\left(1-q_{n}-q_{r}\right)-c_{r}\right) q_{r}+\lambda_{1}\left(q_{n}-q_{r}\right)+\lambda_{2} q_{r} \\
& \partial L_{M}^{N} / \partial q_{n}=1-2 q_{n}-2 \delta q_{r}-\omega-c_{o}+\lambda_{1}=0 \\
& \partial L_{M}^{N} / \partial q_{r}=\delta-c_{r}-2 \delta q_{n}-2 \delta q_{r}-\lambda_{1}+\lambda_{2}=0 \\
& \lambda_{1}\left(q_{n}-q_{r}\right)=\lambda_{2} q_{r}=0 \\
& q_{n}-q_{r} \geq 0, q_{r} \geq 0
\end{aligned}
$$

where $\lambda_{1}$ and $\lambda_{2}$ are the Lagrangian multipliers. According to the Karush-Kuhn-Tucker condition, four possible cases exist for the optimal solutions as per different combinations of nonnegative Lagrangian coefficients $\lambda_{1}$ and $\lambda_{2}$ as follows.

Case I: When $\lambda_{1}=0$ and $\lambda_{2}>0$, the optimal solutions are $q_{n}^{N-A}=\frac{1-c_{o}+c_{r}-\delta-\omega-\lambda_{2}}{2(1-\delta)}$ and $q_{r}^{N-A}=\frac{\delta c_{o}-c_{r}+w \delta+\lambda_{2}}{2 \delta(1-\delta)}$. Then, $q_{n}^{N-A}-q_{r}^{N-A}>0$ and $q_{r}^{N-A}=0$ can be derived based on the Complementary Relaxation Theorem. It is easy to get $\lambda_{2}=c_{r}-\delta\left(c_{o}+\omega\right)$ given $q_{r}^{N-A}=0$. By substituting it into $q_{n}^{N-A}$, one has $q_{n}^{N-A}=\frac{1}{2}\left(1-c_{o}-\omega\right)$. Furthermore, $c_{r}>\delta\left(\omega+c_{o}\right)$ is derived from $\lambda_{2}=c_{r}-\delta\left(c_{o}+\omega\right)>0$, corresponding to the condition for Case I and denoted by $N$ - $A$. This condition $c_{r}>\delta\left(\omega+c_{o}\right)$ can be equivalently expressed as $\omega<\frac{c_{r}}{\delta}-c_{o}$.

Case II: When $\lambda_{1}=0$ and $\lambda_{2}=0$, the optimal solutions are $q_{n}^{N-B}=\frac{1-c_{o}+c_{r}-\delta-\omega}{2(1-\delta)}$ and $q_{r}^{N-B}=\frac{\delta\left(c_{o}+\omega\right)-c_{r}}{2 \delta(1-\delta)}$. $q_{n}^{N-B}-q_{r}^{N-B} \geq 0$ and $q_{r}^{N-B} \geq 0$ can then be obtained from the Complementary Relaxation Theorem. Therefore, we obtain the condition for Case II as $\frac{2 \delta\left(c_{o}+\omega+\delta\right)}{1-\delta}-\delta \leq c_{r} \leq \delta\left(c_{o}+\omega\right)$, denoted by $N$ - $B$, which can be rewritten as $\frac{c_{r}}{\delta}-c_{o} \leq \omega \leq \frac{\left(c_{r}+\delta\right)(1-\delta)+2 \delta c_{r}}{2 \delta}-c_{o}$.

Case III: If $\lambda_{1}>0$ and $\lambda_{2}=0$, the optimal solutions are $q_{n}^{N-C}=\frac{1-c_{o}+c_{r}-\delta-\omega+2 \lambda_{1}}{2(1-\delta)}$ and $q_{r}^{N-C}=\frac{\delta c_{o}-c_{r}+\delta \omega-\lambda_{1}(1+\delta)}{2 \delta(1-\delta)} \cdot q_{n}^{N-C}-q_{r}^{N-C}=0$ and $q_{r}^{N-C}>0$ can be confirmed by the Complementary Relaxation Theorem. 
Solving equation $q_{n}^{N-C}-q_{r}^{N-C}=0$ yields $\lambda_{1}=\frac{\delta\left(\delta-1+2 c_{o}+2 \omega\right)-c_{r}(1+\delta)}{1+3 \delta}$. Substituting it into $q_{n}^{N-C}$ and $q_{r}^{N-C}$ results in $q_{n}^{N-C}=q_{r}^{N-C}=\frac{1-c_{o}-c_{r}-\omega+\delta}{2(1+3 \delta)}$. As $q_{r}^{N-C}>0$, one has the condition for Case III as $c_{r}<\frac{2 \delta\left(c_{o}+\omega+\delta\right)}{1-\delta}-\delta$, denoted by $N-C$, which can be rearranged as $\omega>\frac{\left(c_{r}+\delta\right)(1-\delta)+2 \delta c_{r}}{2 \delta}-c_{o}$.

Case IV: If $\lambda_{1}>0$ and $\lambda_{2}>0, q_{n}^{N-D}=q_{r}^{N-D}$ and $q_{r}^{N-D}=0$ can be verified by the Complementary Relaxation Theorem, which means a case without any production activity and, hence, can be neglected.

According to backward induction, substituting $q_{n}^{N-B}=\frac{1-c_{o}+c_{r}-\delta-\omega}{2(1-\delta)}$ and $q_{r}^{N-B}=\frac{\delta\left(c_{o}+\omega\right)-c_{r}}{2 \delta(1-\delta)}$ into the constraints $q_{n}^{N-B}-q_{r}^{N-B} \geq 0$ and $q_{r}^{N-B} \geq 0$, we obtain the new constraints. Thereafter, the Lagrangian and the Karush-Kuhn-Tucker optimality conditions for the supplier's optimization problem are stated as follows:

$$
\begin{aligned}
& L_{S}^{N-B}=\left(\omega-c_{k}\right) q_{n}+\lambda_{3}\left(\frac{1-c_{o}+c_{r}-\delta-\omega}{2(1-\delta)}-\frac{\delta\left(c_{o}+\omega\right)-c_{r}}{2 \delta(1-\delta)}\right)+\lambda_{4}\left(\frac{\delta\left(c_{o}+\omega\right)-c_{r}}{2 \delta(1-\delta)}\right), \\
& \frac{\partial L_{S}^{N-B}}{\partial w}=\frac{1+c_{k}-c_{o}+c_{r}-2 \omega-\delta-2 \lambda_{3}+\lambda_{4}}{2(1-\delta)}=0, \\
& \lambda_{3}\left(\frac{1-c_{o}+c_{r}-\delta-\omega}{2(1-\delta)}-\frac{\delta\left(c_{o}+\omega\right)-c_{r}}{2 \delta(1-\delta)}\right)=0, \\
& \lambda_{4}\left(\frac{\delta\left(c_{o}+\omega\right)-c_{r}}{2 \delta(1-\delta)}\right)=0 \\
& \frac{1-c_{o}+c_{r}-\delta-\omega}{2(1-\delta)}-\frac{\delta\left(c_{o}+\omega\right)-c_{r}}{2 \delta(1-\delta)} \geq 0, \frac{\delta\left(c_{o}+\omega\right)-c_{r}}{2 \delta(1-\delta)} \geq 0 .
\end{aligned}
$$

There exist four possible cases for the optimal wholesale price given different combinations of nonnegative Lagrange coefficients $\lambda_{3}$ and $\lambda_{4}$.

Case I: If $\lambda_{3}>0$ and $\lambda_{4}>0, \frac{1-c_{o}+c_{r}-\delta-\omega}{2(1-\delta)}=\frac{\delta\left(c_{o}+\omega\right)-c_{r}}{2 \delta(1-\delta)}=0$ is derived by the Complementary Relaxation Theorem. As it is contradictory to $\frac{\delta\left(1-c_{o}-\omega\right)(1-\delta)}{1+\delta}>0$, this case does not exist.

Case II: When $\lambda_{3}=0$ and $\lambda_{4}>0$, the optimal solutions are $\omega^{N-B}=\frac{1+c_{k}-c_{o}+c_{r}-\delta+\lambda_{4}}{2}$ and $q_{r}^{N-B}=\frac{\delta\left(c_{o}+\omega\right)-c_{r}}{2 \delta(1-\delta)}=0 . \quad q_{n}^{N-B}-q_{r}^{N-B}=\frac{1-c_{o}+c_{r}-\delta-\omega}{2(1-\delta)}-\frac{\delta\left(c_{o}+\omega\right)-c_{r}}{2 \delta(1-\delta)}>0 \quad$ can be derived by the Complementary Relaxation Theorem. Solving equation $\frac{\delta\left(c_{o}+\omega\right)-c_{r}}{2 \delta(1-\delta)}=0$ yields $\omega^{N-B-1}=\frac{c_{r}}{\delta}-c_{o}$ and $\lambda_{4}=\frac{2 c_{r}-\delta\left(1+c_{k}+c_{o}+c_{r}\right)+\delta^{2}}{\delta}$. The condition for Case II $\frac{\delta\left(1+c_{k}+c_{o}-\delta\right)}{2-\delta}<c_{r}<\delta$ is due to $q_{n}^{N-B}-q_{r}^{N-B}>0$ and $\lambda_{4}>0$.

Case III: If $\lambda_{3}=0$ and $\lambda_{4}=0$, the optimal solution is $\omega^{N-B-2}=\frac{1+c_{k}-c_{o}+c_{r}-\delta}{2} \cdot q_{n}^{N-B}-q_{r}^{N-B} \geq 0$ and $q_{r}^{N-B} \geq 0$ can be confirmed by the Complementary Relaxation Theorem. Therefore, the condition for Case III is $\delta\left(c_{k}+c_{o}\right) \leq c_{r} \leq \frac{\delta\left(1+c_{k}+c_{o}-\delta\right)}{2-\delta}$.

Case IV: When $\lambda_{3}>0$ and $\lambda_{4}=0$, the optimal solution is $\omega^{N-B}=\frac{1+c_{k}-c_{o}+c_{r}-\delta-2 \lambda_{3}}{2} \cdot q_{n}^{N-B}-q_{r}^{N-B}=0$ and $q_{r}^{N-B} \geq 0$ can be verified by the Complementary Relaxation Theorem. It is easy to get that $\lambda_{3}=\frac{\delta\left(c_{k}+c_{o}\right)-c_{r}}{2 \delta}$ due to 
$q_{n}^{N-B}-q_{r}^{N-B}=0$, and $\omega^{N-B-2}=\frac{c_{r}+\delta-2 \delta c_{o}+\delta c_{r}-\delta^{2}}{2 \delta}$. As $\lambda_{3}>0$ and $q_{r}^{N-B} \geq 0$, the condition for Case IV is obtained as $c_{r}<\delta\left(c_{k}+c_{o}\right)$.

Let $O, A, B$, and $C$ be four points on the $c_{r}$-axis as shown in Figure A.1, where $O: c_{r}=0, A: c_{r}=\delta\left(c_{k}+c_{o}\right), B: c_{r}$ $=\frac{\delta\left(1+c_{k}+c_{o}-\delta\right)}{2-\delta}$, and $C: c_{r}=\delta$. The two intervals $O A$ and $B C$ correspond to the tight constraints $q_{n}^{N-B}-q_{r}^{N-B}=0$ and $q_{r}^{N-B}=0$, respectively. The interval $A B$ corresponds to the loose constraint $0<q_{r}^{N-B}<q_{n}^{N-B}$.

According to backward induction, substituting $q_{n}^{N-A}=\frac{1-\omega-c_{o}}{2}$ and $q_{r}^{N-A}=0$ into the constraints $q_{n}^{N-A}-q_{r}^{N-A} \geq 0$ and $q_{r}^{N-A} \geq 0$, one obtains $\omega \leq 1-c_{o}$. Plugging this new constraint into the supplier's profit function (1), we can obtain the optimal solution $\omega^{N-A}=\frac{1+c_{k}-c_{o}}{2}$ if $c_{r}>\frac{\delta}{2}\left(1+c_{k}+c_{o}\right)$. Denoting point $P: x=\frac{\delta}{2}\left(1+c_{k}+c_{o}\right)$, it is apparent that point $P$ lies between points $B$ and $C$ due to $\frac{\delta\left(1+c_{k}+c_{o}-\delta\right)}{2-\delta}<\frac{\delta}{2}\left(1+c_{k}+c_{o}\right)<\delta$.

By backward induction, substituting $q_{n}^{N-C}=q_{r}^{N-C}=\frac{1-\omega-c_{o}-c_{r}+\delta}{2(1+3 \delta)}$ into the constraints $q_{n}^{N-C}-q_{r}^{N-C} \geq 0$ and $q_{r}^{N-C} \geq 0$, one obtains the new constraint condition $\omega \leq 1-c_{o}-c_{r}+\delta$. With the new constraint, the equilibrium decision is derived as $\omega^{N-C}=\frac{1+c_{k}-c_{o}-c_{r}+\delta}{2}$ if $0<c_{r}<\frac{\delta}{1+2 \delta}\left(2 \delta+c_{k}+c_{o}\right)$. Denoting point $X: c_{r}=\frac{\delta}{1+2 \delta}\left(2 \delta+c_{k}+c_{o}\right)$, we know that point $P$ lies between points $B$ and $C$, but the relationship between point $X$ and point $P$ is uncertain due to $\frac{\delta}{1+2 \delta}\left(2 \delta+c_{k}+c_{o}\right)<\frac{\delta}{2}\left(1+c_{k}+c_{o}\right)<\delta$ and $\delta\left(c_{k}+c_{o}\right)<\frac{\delta}{1+2 \delta}\left(2 \delta+c_{k}+c_{o}\right)<\delta$.

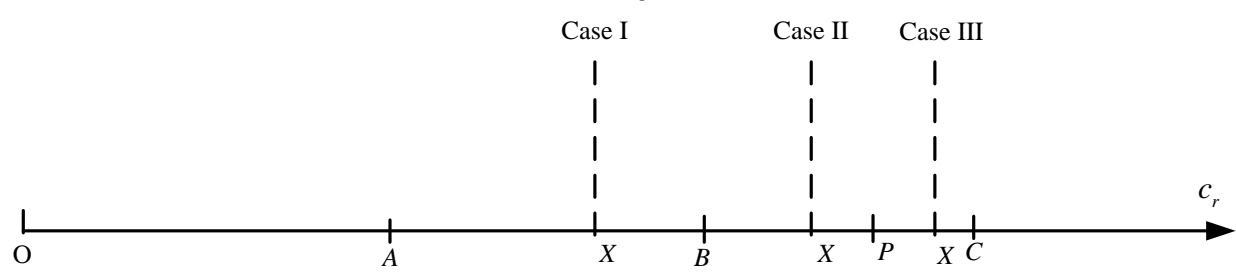

Figure A.1. The location of each boundary point on the $c_{r}$-axis.

It is understandable that the supplier's profit function takes different forms when $c_{r}$ is located in different segments of values as shown in Fig. A.1. Let $\prod_{M N}\left(c_{r}\right)$ denote the supplier's profit function when $c_{r}$ falls in the interval between point $M$ and $N$, corresponding to different constraints in the manufacturer's optimal decisions. In the interval $O A(P C)$, $\prod_{O X}\left(c_{r}\right)-\prod_{O A}\left(c_{r}\right)>0$ for $c_{r}<\delta\left(c_{k}+c_{o}\right) \quad\left(\prod_{P C}\left(c_{r}\right)-\prod_{B C}\left(c_{r}\right)>0\right.$ for $\left.\frac{\delta}{2}\left(1+c_{k}+c_{o}\right)<c_{r}<\delta\right)$ means that when $c_{r}$ is small (large) enough to fall within the range in case $N-C(N-A)$, the supplier can enjoy higher profit under the loose constraint.

Depending on where $X$ is located, the following three possible cases may arise:

Case I: If $0<\delta<1 / 3, X$ lies between point $A$ and $B$.

In this case, $\prod_{O X}\left(c_{r}\right)-\prod_{O A}\left(c_{r}\right)>0$ always holds for interval $O A c_{r}<\delta\left(c_{k}+c_{o}\right)$. When $c_{r}$ falls between $A$ and $X$, solving $\prod_{O X}\left(c_{r}\right)-\prod_{A B}\left(c_{r}\right)=0$ for $c_{r}$, we obtain $c_{r}=x_{1}^{1}$ or $c_{r}=x_{1}^{2}$ where $x_{1}^{1}<A<x_{1}^{2}<X<B$. It is apparent that $c_{r}=x_{1}^{1}$ should be discarded as it is outside the interval $A X$. In addition, $\prod_{O X}\left(c_{r}\right)-\prod_{A B}\left(c_{r}\right)>0$ for $\delta\left(c_{k}+c_{o}\right)<c_{r}<x_{1}^{2}$ and $\prod_{O X}\left(c_{r}\right)-\prod_{A B}\left(c_{r}\right)<0$ for $x_{1}^{2}<c_{r}<X \quad$ can be confirmed easily given $0<\delta<1 / 3 \quad, \quad$ where $x_{1}^{2}=\delta-\frac{(1+\delta-\sqrt{(1+3 \delta)(1-\delta)})\left(1-c_{k}-c_{o}\right)}{2 \delta}$. 
Therefore, in Case I, the new component pricing decisions are stated as follows:

1) $\omega^{N-A}=\frac{1}{2}\left(1+c_{k}-c_{o}\right)$, if $P<c_{r}<C$.

2) $\omega^{N-B-1}=\frac{c_{r}}{\delta}-c_{o}$, if $B<c_{r}<P$.

3) $\omega^{N-B-2}=\frac{1}{2}\left(1+c_{k}-c_{o}+c_{r}-\delta\right)$, if $x_{1}^{2}<c_{r}<B$.

4) $\omega^{N-C}=\frac{1}{2}\left(1+c_{k}-c_{o}-c_{r}+\delta\right)$, if $O<c_{r}<x_{1}^{2}$.

Case II: If $1 / 3 \leq \delta<1 / 2$, point $X$ lies between point $B$ and $P$. For interval $O A$, Case I already confirms that $\prod_{O X}\left(c_{r}\right)-\prod_{O A}\left(c_{r}\right)>0$ always holds. For interval $A B, \prod_{O X}\left(c_{r}\right)-\prod_{A B}\left(c_{r}\right)>0$ if $A<c_{r}<x_{1}^{2}$ and $\prod_{O X}\left(c_{r}\right)-\prod_{A B}\left(c_{r}\right)<0$ when $x_{1}^{2}<c_{r}<B$. In interval $B P$, solving $\prod_{O X}\left(c_{r}\right)-\prod_{B P}\left(c_{r}\right)=0$ for $c_{r}$ yields $c_{r}=x_{2}^{1}$ or $c_{r}=x_{2}^{2}$, where $x_{2}^{1}=\delta-\frac{(2+5 \delta+2 \sqrt{2 \delta(1+3 \delta)})\left(1-c_{k}-c_{o}\right)}{4(1+3 \delta)+\delta^{2}}$. Due to $1 / 3<\delta<1 / 2, \quad x_{2}^{1}<B<X<P<x_{2}^{2}$ holds, implying that $\prod_{O X}\left(c_{r}\right)-\prod_{B P}\left(c_{r}\right)<0$ when $B<c_{r}<P$.

Therefore, in Case II, the new component pricing decisions are the same as those in case I.

Case III: If $1 / 2<\delta<1$, point $X$ lies between point $P$ and $C$.

The optimal solution is divided into two subcases:

The first subcase III_I: When $1 / 2<\delta<2 / 3, \prod_{O X}\left(c_{r}\right)-\prod_{A B}\left(c_{r}\right)>0$ if $A<c_{r}<x_{1}^{2}$ and $\prod_{O X}\left(c_{r}\right)-\prod_{A B}\left(c_{r}\right)<0$ when $x_{1}^{2}<c_{r}<B$. In interval $B P$, solving $\prod_{O X}\left(c_{r}\right)-\prod_{B P}\left(c_{r}\right)=0$ for $c_{r}$ yields $c_{r}=x_{3}^{1}$ or $c_{r}=x_{3}^{2}$, where $x_{3}^{1}<P<X<x_{3}^{2}$ holds. This means that $\prod_{O X}\left(c_{r}\right)-\prod_{B C}\left(c_{r}\right)<0$ when $B<c_{r}<P$. In this case, the new component pricing decisions are the same as those in case I.

The second subcase III_II: When $2 / 3<\delta<1, \quad x_{1}^{1}<A<B<x_{2}^{2}$ and $B<x_{2}^{1}<P<X<x_{2}^{2}$ hold, indicating that $\prod_{O X}\left(c_{r}\right)-\prod_{A B}\left(c_{r}\right)>0$ for $A<c_{r}<B$ and $\prod_{A X}-\prod_{B C}<0$ for $B<c_{r}<C$. In this case, the new component pricing decisions are stated as follows:

1) $\omega^{N-A}=\frac{1}{2}\left(1+c_{k}-c_{o}\right)$, if $P<c_{r}<C$.

2) $\omega^{N-B-2}=\frac{1}{2}\left(1+c_{k}-c_{o}+c_{r}-\delta\right)$, if $x_{2}^{1}<c_{r}<P$.

3) $\omega^{N-C}=\frac{1}{2}\left(1+c_{k}-c_{o}-c_{r}+\delta\right)$, if $O<c_{r}<x_{2}^{1}$.

In summary, if $0<\delta<2 / 3, X$ can fall within any segment between $A$ and $C$. Under this scenario, $A<x_{1}^{2}<B$ holds and an optimal wholesale price exists. If $2 / 3<\delta<1$, point $X$ must fall between $P$ and $C$. Under this circumstance, $B<x_{2}^{1}<P$ holds and another optimal wholesale price exists.

Let $c_{r 1}^{N}=x_{1}^{2}, \quad c_{r 2}^{N}=B, \quad c_{r 3}^{N}=x_{2}^{1}, \quad c_{r 4}^{N}=P$, and $\operatorname{com}\left\{c_{r 2}^{N}, c_{r 3}^{N}\right\}=\left\{\begin{array}{ll}c_{r 1}^{N} & \text { if } c_{r 2}^{N} \geq c_{r 3}^{N} \\ c_{r 3}^{N} & \text { if } c_{r 2}^{N}<c_{r 3}^{N}\end{array}\right.$, the supplier's component pricing decisions with respect to the remanufacturing cost are stated as follows:

Case $N-A: \quad \omega^{N-A}=\frac{1}{2}\left(1+c_{k}-c_{o}\right)$, if $c_{r}>c_{r 4}^{N} ;$

Case $N-B-1: \quad \omega^{N-B-1}=\frac{c_{r}}{\delta}-c_{o}$, if $\max \left\{c_{r 2}^{N}, c_{r 3}^{N}\right\} \leq c_{r} \leq c_{r 4}^{N}$;

Case $N-B-2: \quad \omega^{N-B-2}=\frac{1}{2}\left(1+c_{k}-c_{o}+c_{r}-\delta\right)$, if $c_{r 1}^{N} \leq c_{r}<c_{r 2}^{N}$; 
Case $N-C: \quad \omega^{N-C}=\frac{1}{2}\left(1+c_{k}-c_{o}-c_{r}+\delta\right)$, if $0<c_{r}<\operatorname{com}\left\{c_{r 2}^{N}, c_{r 3}^{N}\right\}$

Equilibrium solution under scenario $N$ is thus verified.

\section{Appendix E. Proof of equilibrium solution under scenario $M$}

The proofs of equilibrium solution under scenario $M$ can be carried out in a similar fashion as those under scenario $N$ in Appendix D.

$$
\begin{aligned}
& \text { Here, } \quad c_{r 1}^{M}=\delta-\frac{\left(1-c_{k}-c_{o}\right)\left(4 \delta \beta_{o}-\Delta_{o}\left(\Delta_{o}-\eta_{o}\right)\right)\left(2 \delta \beta_{o}-\Delta_{o}^{2}\right)}{\left(2 \beta_{o}(1+\delta)+\eta_{o}\left(\Delta_{o}-\eta_{o}\right)\right)\left(2 \delta \beta_{o}-\Delta_{o}^{2}\right)-\sqrt{2 \beta_{o}\left(2 \beta_{o}(1+3 \delta)-\left(\Delta_{o}-\eta_{o}\right)^{2}\right)\left(2 \delta \beta_{o}-\Delta_{o}^{2}\right)\left(2 \delta \beta_{o}(1-\delta)-\delta \eta_{o}^{2}-2 \delta \eta_{o} \Delta_{o}-\Delta_{o}^{2}\right)}}, \\
& c_{r 2}^{M}=\delta-\frac{\left(1-c_{k}-c_{o}\right)\left(2 \beta_{o} \delta+\eta_{o} \Delta_{o}^{2}\right)\left(2 \delta \beta_{o}-\Delta_{o}^{2}\right)}{4 \delta \beta_{o}\left(\beta_{o}(2-\delta)-\eta_{o}^{2}\right)-4 \delta \eta_{o} \beta_{o} \Delta_{o}-\Delta_{o}^{2}\left(4 \beta_{o}-\eta_{o}^{2}\right)} \quad, \quad c_{r 4}^{M}=\delta-\frac{\left(1-c_{k}-c_{o}\right)\left(2 \delta \beta_{o}+\eta_{o} \Delta_{o}\right)}{2\left(2 \beta_{o}-\eta_{o}^{2}\right)} \\
& c_{r 3}^{M}=\delta-\frac{\left(1-c_{k}-c_{o}\right)\left(2 \beta_{o} \delta+\eta_{o} \Delta_{o}\right)^{2}}{\left(2 \delta \beta_{o}+\eta_{o} \Delta_{o}\right)\left(2 \beta_{o}(2+5 \delta)-2 \eta_{o}^{2}+3 \eta_{o} \Delta_{o}-2 \Delta_{o}^{2}\right)-2 \sqrt{\left(2 \beta_{o}(1+3 \delta)-\left(\Delta_{o}-\eta_{o}\right)^{2}\right)\left(2 \delta \beta_{o}+\eta_{o} \Delta_{o}\right)^{2}\left(4 \beta_{o} \delta-\Delta_{o}\left(\Delta_{o}-\eta_{o}\right)\right)}} \\
& \operatorname{com}\left\{c_{r 2}^{M}, c_{r 3}^{M}\right\}=\left\{\begin{array}{ll}
c_{r 1}^{M} & \text { if } c_{r 2}^{M} \geq c_{r 3}^{M} \\
c_{r 3}^{M} & \text { if } c_{r 2}^{M}<c_{r 3}^{M}
\end{array} .\right.
\end{aligned}
$$

\section{Appendix F. Proof of equilibrium solution under scenario $S$}

The proofs of equilibrium solution under scenario $S$ can be carried out in a similar fashion as those under scenario $N$ in Appendix D.

Here,

$$
c_{r 1}^{S}=\delta-\frac{\left(1-c_{k}-c_{o}\right)\left(4 \beta_{k}(1+\delta)-\eta_{k}^{2}-\Delta_{k}^{2}+\sqrt{\left(4 \beta_{k}(1+3 \delta)-\left(\eta_{k}-\Delta_{k}\right)^{2}\right)\left(4 \beta_{k}(1-\delta)-\left(\eta_{k}+\Delta_{k}\right)^{2}\right)}\right)}{8 \beta_{k} \delta+2 \eta_{k} \Delta_{k}}
$$

$c_{r 2}^{S}=\delta-\frac{\left(1-c_{k}-c_{o}\right)\left(2 \beta_{k} \delta+\Delta_{k}\left(\eta_{k}+\Delta_{k}\right)\right)}{2 \beta_{k}(2-\delta)-\eta_{k}\left(\eta_{k}+\Delta_{k}\right)}$

$$
c_{r 4}^{S}=\delta-\frac{\left(1-c_{k}-c_{o}\right)\left(2 \beta_{k} \delta+\eta_{k} \Delta_{k}\right)}{4 \beta_{k}-\eta_{k}^{2}}
$$

$c_{r 3}^{S}=\delta-\left(1-c_{k}-c_{o}\right)\left(\begin{array}{c}\frac{\left(2 \beta_{k} \delta\left(2 \beta_{k}(2+5 \delta)-\eta_{k}^{2}\right)+\eta_{k} \Delta_{k}\left(4 \beta_{k}(1+3 \delta)-\left(\eta_{k}-\Delta_{k}\right)^{2}\right)-2 \beta_{k}(2+\delta) \Delta_{k}^{2}\right)}{4 \beta_{k}\left(\beta_{k}(4+\delta(12+\delta))+\eta_{k} \Delta_{k}(2+\delta)\right)-\eta_{k}^{2}\left(4 \beta_{k}(2+3 \delta)-\left(\eta_{k}-\Delta_{k}\right)^{2}\right)} \\ +\frac{\left.4 \sqrt{2 \beta_{k}^{2} \delta\left(4 \beta_{k}(1+3 \delta)-\left(\eta_{k}-\Delta_{k}\right)^{2}\right)\left(\beta_{k} \delta^{2}+\Delta_{k}\left(\eta_{k} \delta+\Delta_{k}\right)\right.}\right)}{4 \beta_{k}\left(\beta_{k}(4+\delta(12+\delta))+\eta_{k} \Delta_{k}(2+\delta)\right)-\eta_{k}^{2}\left(4 \beta_{k}(2+3 \delta)-\left(\eta_{k}-\Delta_{k}\right)^{2}\right)}\end{array}\right)$, and $\operatorname{com}\left\{c_{r 2}^{S}, c_{r 3}^{S}\right\}=\left\{\begin{array}{ll}c_{r 1}^{s} & \text { if } c_{r 2}^{S} \geq c_{r 3}^{S} \\ c_{r 3}^{S} & \text { if } c_{r 2}^{S}<c_{r 3}^{S}\end{array}\right.$.

\section{Appendix G. Proof of Proposition 2}

Under scenario $N$, comparing $c_{r 2}^{N}$ with $c_{r 3}^{N}$, one can confirm that $c_{r 2}^{N}-c_{r 3}^{N}=\delta\left(1-c_{k}-c_{o}\right)\left(\frac{1}{2+5 \delta-2 \sqrt{2 \delta(1+3 \delta)}}-\frac{1}{2-\delta}\right)$. When $\delta>\bar{\delta}_{N}=\frac{2}{3}, c_{r 2}^{N}$ is less than $c_{r 3}^{N}$ and case $N-B-2$ disappears. As such, the manufacturer is left with two remanufacturing strategies: no and complete remanufacturing. On the other hand, if $\delta<\bar{\delta}_{N}, c_{r 2}^{N}$ is larger than $c_{r 3}^{N}$ and case $N$-B-2 arises. In this case, the manufacturer has three remanufacturing strategies: no, partial and complete remanufacturing. The proofs for scenario $M$ and $S$ can be carried out similarly with different thresholds $\bar{\delta}_{M}=\frac{1}{36 \beta_{o}}\left(\Phi_{1}+\frac{\Phi_{2}}{\Phi_{3}}+\Phi_{3}\right)<\bar{\delta}_{N}$ and $\bar{\delta}_{S}=\frac{4 \beta_{k}-\left(\Delta_{k}+\eta_{k}\right)^{2}-2 \Delta_{k} \eta_{k}+\sqrt{\left(4 \beta_{k}-\left(\Delta_{k}+\eta_{k}\right)^{2}-2 \Delta_{k} \eta_{k}\right)-48 \beta_{k} \Delta_{k}^{2}}}{12 \beta_{k}}<\bar{\delta}_{N} \quad, \quad$ where $\quad \Phi_{1}=8 \beta_{o}-4 \eta_{o}^{2}-13 \eta_{o} \Delta_{o}+5 \Delta_{o}^{2}$, 


$$
\begin{aligned}
& \Phi_{2}=\beta_{o}^{2}\left(8 \beta_{o}-4 \eta_{o}^{2}-\eta_{o} \Delta_{o}-\Delta_{o}^{2}\right)\left(8 \beta_{o}-4 \eta_{o}^{2}-25 \eta_{o} \Delta_{o}-25 \Delta_{o}^{2}\right) \quad, \quad \Phi_{3}=\left(\Phi_{31}+\Phi_{32}\right)^{\frac{1}{3}} \\
& \Phi_{31}=36 \beta_{o}^{6} \Delta_{o}\left(\eta_{o}+\Delta_{o}\right) \sqrt{3 \Delta_{o}\left(\eta_{o}+\Delta_{o}\right)\left(2 \beta_{o}-\eta_{o}^{2}\right)\left(92 \eta_{o} \Delta_{o}\left(2 \beta_{o}-\eta_{o}^{2}\right)+\left(184 \beta_{o}+33 \eta_{o}^{2}\right) \Delta_{o}^{2}+125 \Delta_{o}^{3}\left(2 \eta_{o}+\Delta_{o}\right)-16\left(2 \beta_{o}-\eta_{o}^{2}\right)^{2}\right)} \quad, \\
& \Phi_{32}=\beta_{o}^{6}\left(64\left(2 \beta_{o}-\eta_{o}^{2}\right)^{3}-624\left(2 \beta_{o}-\eta_{o}^{2}\right)^{2} \Delta_{o} \eta_{o}-12\left(104 \beta_{o}-149 \eta_{o}^{2}\right)\left(2 \beta_{o}-\eta_{o}^{2}\right) \Delta_{o}^{2}+\left(4656 \beta_{o}-2203 \eta_{o}^{2}\right) \eta_{o} \Delta_{o}^{3}+3\left(776 \beta_{o}-263 \eta_{o}^{2}\right) \Delta_{o}^{4}+\Delta_{o}^{5}\left(125 \Delta_{o}+375 \eta_{o}\right) .\right.
\end{aligned},
$$

Proposition 2 is thus proved.

\section{Appendix H. Proof of Result 1}

By examining the relevant first order partial derivatives, we derive the sensitivity analysis result of the equilibrium product design decisions on model parameters $c_{r}, c_{k}$, and $c_{o}$ as follows:

(1) $\frac{\partial \gamma_{o}^{M-A}}{\partial c_{r}}=\frac{\partial \gamma_{k}^{M-A}}{\partial c_{r}}=0 ; \frac{\partial \gamma_{o}^{M-A}}{\partial c_{k}}=\frac{\partial \gamma_{o}^{M-A}}{\partial c_{o}}=\frac{\eta_{o}}{2\left(2 \beta_{o}-\eta_{o}^{2}\right)}>0$ and $\frac{\partial \gamma_{k}^{S-A}}{\partial c_{k}}=\frac{\partial \gamma_{k}^{S-A}}{\partial c_{o}}=\frac{\eta_{k}}{4 \beta_{k}-\eta_{k}^{2}}>0$;

(2) $\frac{\partial \gamma_{o}^{M-B-1}}{\partial c_{r}}=\frac{\eta_{o}}{2 \delta \beta_{o}+\eta_{o} \Delta_{o}}>0 \quad, \quad \frac{\partial \gamma_{k}^{S-B-1}}{\partial c_{r}}=\frac{\delta \eta_{k}+2 \Delta_{k}}{2\left(\beta_{k} \delta^{2}+\Delta_{k}\left(\delta \eta_{k}+\Delta_{k}\right)\right)}>0 \quad ; \quad \frac{\partial \gamma_{o}^{M-B-1}}{\partial c_{k}}=0$ $\frac{\partial \gamma_{k}^{S-B-1}}{\partial c_{k}}=-\frac{\delta \Delta_{k}}{2\left(\beta_{k} \delta^{2}+\Delta_{k}\left(\delta \eta_{k}+\Delta_{k}\right)\right)}<0 ; \frac{\partial \gamma_{o}^{M-B-1}}{\partial c_{o}}=0$, and $\frac{\partial \gamma_{k}^{S-B-1}}{\partial c_{o}}=-\frac{\delta \Delta_{k}}{2\left(\beta_{k} \delta^{2}+\Delta_{k}\left(\delta \eta_{k}+\Delta_{k}\right)\right)}<0$

(3) $\frac{\partial \gamma_{o}^{M-B-2}}{\partial c_{r}}=-\frac{1}{2}\left(\frac{\Delta_{o}}{2 \delta \beta_{o}-\Delta_{o}^{2}}+\frac{\delta \eta_{o}+\Delta_{o}}{2 \delta \beta_{o}(1-\delta)-\left(\delta \eta_{o}^{2}+2 \delta \eta_{o} \Delta_{o}+\Delta_{o}^{2}\right)}\right)<0 \quad, \quad \frac{\partial \gamma_{k}^{S-B-2}}{\partial c_{r}}=-\frac{\eta_{k}+\Delta_{k}}{4 \beta_{k}(1-\delta)-\left(\eta_{k}+\Delta_{k}\right)^{2}}<0$ $\left.\frac{\partial \gamma_{o}^{M-B-2}}{\partial c_{k}}=\frac{\partial \gamma_{o}^{M-B-2}}{\partial c_{o}}=\frac{\delta\left(\eta_{o}+\Delta_{o}\right)}{2 \delta \beta_{o}(1-\delta)-\left(\delta \eta_{o}^{2}+2 \delta \eta_{o} \Delta_{o}+\Delta_{o}^{2}\right)}\right)>0$, and $\frac{\partial \gamma_{k}^{S-B-2}}{\partial c_{k}}=\frac{\partial \gamma_{k}^{S-B-2}}{\partial c_{o}}=\frac{\eta_{k}+\Delta_{k}}{4 \beta_{k}(1-\delta)-\left(\eta_{k}+\Delta_{k}\right)^{2}}>0$

(4) $\frac{\partial \gamma_{o}^{M-C}}{\partial c_{r}}=\frac{\partial \gamma_{o}^{M-C}}{\partial c_{k}}=\frac{\partial \gamma_{o}^{M-C}}{\partial c_{o}}=-\frac{\Delta_{o}-\eta_{o}}{2\left(2 \beta_{o}(1+3 \delta)-\left(\Delta_{o}-\eta_{o}\right)^{2}\right)}$, and $\frac{\partial \gamma_{k}^{S-C}}{\partial c_{r}}=\frac{\partial \gamma_{k}^{S-C}}{\partial c_{k}}=\frac{\partial \gamma_{k}^{S-C}}{\partial c_{o}}=-\frac{\Delta_{k}-\eta_{k}}{4 \beta_{k}(1+3 \delta)-\left(\Delta_{k}-\eta_{k}\right)^{2}}$.

Once again, the optimality conditions $2 \beta_{o}(1+3 \delta)-\left(\Delta_{o}-\eta_{o}\right)^{2}>0$ and $4 \beta_{k}(1+3 \delta)-\left(\Delta_{k}-\eta_{k}\right)^{2}>0$ imply that, if $\Delta_{i}-\eta_{i}>0, \frac{\partial \gamma_{i}^{h-C}}{\partial c_{r}}=\frac{\partial \gamma_{i}^{h-C}}{\partial c_{k}}=\frac{\partial \gamma_{i}^{h-C}}{\partial c_{o}}<0$; otherwise, $\frac{\partial \gamma_{i}^{h-C}}{\partial c_{r}}=\frac{\partial \gamma_{i}^{h-C}}{\partial c_{k}}=\frac{\partial \gamma_{i}^{h-C}}{\partial c_{o}}<0$. Where, $h \in\{M, S\}, i \in\{k, o\}$.

Result 1 is thus confirmed.

\section{Appendix I. Proof of Result 2}

By examining the relevant first order partial derivatives, we obtain the following sensitivity analysis result of the equilibrium decisions with respect to $c_{k}$.

$$
\begin{aligned}
& \text { (1) } \frac{\partial \omega^{N-A}}{\partial c_{k}}=\frac{\partial \omega^{N-B-2}}{\partial c_{k}}=\frac{\partial \omega^{N-C}}{\partial c_{k}}=\frac{1}{2}>0 \quad, \quad \frac{\partial \omega^{M-A}}{\partial c_{k}}=\frac{\partial \omega^{M-B-2}}{\partial c_{k}}=\frac{\partial \omega^{M-C}}{\partial c_{k}}=\frac{1}{2}>0 ; \frac{\partial \omega^{S-A}}{\partial c_{k}}=\frac{2 \beta_{k}}{4 \beta_{k}-\eta_{k}^{2}}>0 \quad \text {, } \\
& \frac{\partial \omega^{S-B-2}}{\partial c_{k}}=\frac{2 \beta_{k}(1-\delta)-\Delta_{k}\left(\eta_{k}+\Delta_{k}\right)}{4 \beta_{k}(1-\delta)-\left(\eta_{k}+\Delta_{k}\right)^{2}}>0 \quad, \quad \frac{\partial \omega^{S-C}}{\partial c_{k}}=\frac{2 \beta_{k}(1+3 \delta)-\Delta_{k}\left(\Delta_{k}-\eta_{k}\right)}{4 \beta_{k}(1+3 \delta)-\left(\Delta_{k}-\eta_{k}\right)^{2}}>0 \quad ; \quad \frac{\partial \omega^{N-B-1}}{\partial c_{k}}=\frac{\partial \omega^{M-B-1}}{\partial c_{k}}=0 \quad, \quad \text { and } \\
& \frac{\partial \omega^{S-B-1}}{\partial c_{k}}=\frac{\Delta_{k}^{2}}{2\left(\beta_{k} \delta^{2}+\Delta_{k}\left(\eta_{k} \delta+\Delta_{k}\right)\right)}>0 \\
& \text { (2) } \frac{\partial q_{n}^{N-A}}{\partial c_{k}}=-\frac{1}{4}<0 \quad, \quad \frac{\partial q_{n}^{M-A}}{\partial c_{k}}=-\frac{\beta_{o}}{2\left(2 \beta_{o}-\eta_{o}^{2}\right)}<0 \quad, \quad \frac{\partial q_{n}^{S-A}}{\partial c_{k}}=-\frac{\beta_{k}}{4 \beta_{k}-\eta_{k}^{2}}<0 \quad ; \quad \frac{\partial q_{n}^{N-B-1}}{\partial c_{k}}=\frac{\partial q_{n}^{M-B-1}}{\partial c_{k}}=0 \quad \text {, } \\
& \frac{\partial q_{n}^{S-B-1}}{\partial c_{k}}=-\frac{\Delta_{k}^{2}}{2\left(\beta_{k} \delta^{2}+\Delta_{k}\left(\eta_{k} \delta+\Delta_{k}\right)\right)}<0 ; \frac{\partial q_{n}^{N-B-2}}{\partial c_{k}}=-\frac{1}{4(1-\delta)}<0, \frac{\partial q_{n}^{M-B-2}}{\partial c_{k}}=-\frac{2 \delta \beta_{o}-\Delta_{o}^{2}}{4\left(2 \delta \beta_{o}(1-\delta)-\left(\delta \eta_{o}^{2}+2 \delta \eta_{o} \Delta_{o}+\Delta_{o}^{2}\right)\right)}<0,
\end{aligned}
$$


$\frac{\partial q_{n}^{S-B-2}}{\partial c_{k}}=-\frac{\beta_{k}}{4 \beta_{k}(1-\delta)-\left(\eta_{k}+\Delta_{k}\right)^{2}}<0 \quad ; \quad \frac{\partial q_{n}^{N-C}}{\partial c_{k}}=-\frac{1}{4(1+3 \delta)}<0 \quad, \quad \frac{\partial q_{n}^{M-C}}{\partial c_{k}}=-\frac{\beta_{o}}{2\left(2 \beta_{o}(1+3 \delta)-\left(\eta_{o}-\Delta_{o}\right)^{2}\right)}<0 \quad, \quad$ and $\frac{\partial q_{n}^{S-C}}{\partial c_{k}}=-\frac{\beta_{k}}{4 \beta_{k}(1+3 \delta)-\left(\eta_{k}-\Delta_{k}\right)^{2}}<0$.

(3) $\frac{\partial q_{r}^{N-A}}{\partial c_{k}}=\frac{\partial q_{r}^{N-B-1}}{\partial c_{k}}=0 \quad, \quad \frac{\partial q_{r}^{M-A}}{\partial c_{k}}=\frac{\partial q_{r}^{M-B-1}}{\partial c_{k}}=0 \quad, \quad \frac{\partial q_{r}^{S-A}}{\partial c_{k}}=\frac{\partial q_{r}^{S-B-1}}{\partial c_{k}}=0 \quad ; \quad \frac{\partial q_{r}^{N-B-2}}{\partial c_{k}}=\frac{1}{4(1-\delta)}>0$, $\frac{\partial q_{r}^{M-B-2}}{\partial c_{k}}=\frac{2 \delta \beta_{o}+\eta_{o} \Delta_{o}}{4\left(2 \delta \beta_{o}(1-\delta)-\left(\delta \eta_{o}^{2}+2 \delta \eta_{o} \Delta_{o}+\Delta_{o}^{2}\right)\right)}>0, \frac{\partial q_{r}^{S-B-2}}{\partial c_{k}}=\frac{2 \delta \beta_{k}+\Delta_{k}\left(\eta_{k}+\Delta_{k}\right)}{2 \delta\left(4 \beta_{k}(1-\delta)-\left(\eta_{k}+\Delta_{k}\right)^{2}\right)}>0 ; \quad \frac{\partial q_{r}^{N-C}}{\partial c_{k}}=-\frac{1}{4(1+3 \delta)}<0$, $\frac{\partial q_{r}^{M-C}}{\partial c_{k}}=-\frac{\beta_{o}}{2\left(2 \beta_{o}(1+3 \delta)-\left(\eta_{o}-\Delta_{o}\right)^{2}\right)}<0$, and $\frac{\partial q_{r}^{S-C}}{\partial c_{k}}=-\frac{\beta_{k}}{4 \beta_{k}(1+3 \delta)-\left(\eta_{k}-\Delta_{k}\right)^{2}}<0$.

By the following first order partial derivatives, we derive the sensitivity analysis result of the equilibrium decisions with respect to $c_{o}$ as shown below.

(1) $\frac{\partial \omega^{N-A}}{\partial c_{o}}=\frac{\partial \omega^{N-B-2}}{\partial c_{o}}=\frac{\partial \omega^{N-C}}{\partial c_{o}}=-\frac{1}{2}<0 \quad, \quad \frac{\partial \omega^{M-A}}{\partial c_{o}}=\frac{\partial \omega^{M-B-2}}{\partial c_{o}}=\frac{\partial \omega^{M-C}}{\partial c_{o}}=-\frac{1}{2}<0 \quad, \quad \frac{\partial \omega^{S-A}}{\partial c_{o}}=-\frac{2 \beta_{k}-\eta_{k}^{2}}{4 \beta_{k}-\eta_{k}{ }^{2}}<0$ $\frac{\partial \omega^{S-B-2}}{\partial c_{o}}=-\frac{2 \beta_{k}(1-\delta)+\Delta_{k}\left(\eta_{k}+\Delta_{k}\right)}{4 \beta_{k}(1-\delta)-\left(\eta_{k}+\Delta_{k}\right)^{2}}<0, \frac{\partial \omega^{S-C}}{\partial c_{o}}=-\frac{2 \beta_{k}(1+3 \delta)+\eta_{k}\left(\Delta_{k}-\eta_{k}\right)}{4 \beta_{k}(1+3 \delta)-\left(\eta_{k}-\Delta_{k}\right)^{2}}<0 ; \quad \frac{\partial \omega^{N-B-1}}{\partial c_{o}}=\frac{\partial \omega^{M-B-1}}{\partial c_{o}}=-1<0$, and $\frac{\partial \omega^{S-B-1}}{\partial c_{o}}=-\frac{2 \beta_{k} \delta^{2}+\Delta_{k}\left(2 \eta_{k} \delta+\Delta_{k}\right)}{2\left(\beta_{k} \delta^{2}+\Delta_{k}\left(\eta_{k} \delta+\Delta_{k}\right)\right)}<0$.

(2) $\frac{\partial q_{n}^{N-A}}{\partial c_{o}}=-\frac{1}{4}<0 \quad, \quad \frac{\partial q_{n}^{M-A}}{\partial c_{o}}=-\frac{\beta_{o}}{2\left(2 \beta_{o}-\eta_{o}^{2}\right)}<0 \quad, \quad \frac{\partial q_{n}^{S-A}}{\partial c_{o}}=-\frac{\beta_{k}}{4 \beta_{k}-\eta_{k}^{2}}<0 \quad ; \quad \frac{\partial q_{n}^{N-B-1}}{\partial c_{o}}=\frac{\partial q_{n}^{M-B-1}}{\partial c_{o}}=0$, $\frac{\partial q_{n}^{S-B-1}}{\partial c_{o}}=-\frac{\Delta_{k}^{2}}{2\left(\beta_{k} \delta^{2}+\Delta_{k}\left(\eta_{k} \delta+\Delta_{k}\right)\right)}<0 ; \frac{\partial q_{n}^{N-B-2}}{\partial c_{o}}=-\frac{1}{4(1-\delta)}<0, \quad \frac{\partial q_{n}^{M-B-2}}{\partial c_{o}}=-\frac{2 \delta \beta_{o}-\Delta_{o}^{2}}{4\left(2 \delta \beta_{o}(1-\delta)-\left(\delta \eta_{o}^{2}+2 \delta \eta_{o} \Delta_{o}+\Delta_{o}^{2}\right)\right)}<0$, $\frac{\partial q_{n}^{S-B-2}}{\partial c_{o}}=-\frac{\beta_{k}}{4 \beta_{k}(1-\delta)-\left(\eta_{k}+\Delta_{k}\right)^{2}}<0 \quad ; \quad \frac{\partial q_{n}^{N-C}}{\partial c_{o}}=-\frac{1}{4(1+3 \delta)}<0 \quad, \quad \frac{\partial q_{n}^{M-C}}{\partial c_{o}}=-\frac{\beta_{o}}{2\left(2 \beta_{o}(1+3 \delta)-\left(\eta_{o}-\Delta_{o}\right)^{2}\right)}<0 \quad, \quad$ and $\frac{\partial q_{n}^{S-C}}{\partial c_{o}}=-\frac{\beta_{k}}{4 \beta_{k}(1+3 \delta)-\left(\eta_{k}-\Delta_{k}\right)^{2}}<0$.

(3) $\frac{\partial q_{r}^{N-A}}{\partial c_{o}}=\frac{\partial q_{r}^{N-B-1}}{\partial c_{o}}=0 \quad, \quad \frac{\partial q_{r}^{M-A}}{\partial c_{o}}=\frac{\partial q_{r}^{M-B-1}}{\partial c_{o}}=0 \quad, \quad \frac{\partial q_{r}^{S-A}}{\partial c_{o}}=\frac{\partial q_{r}^{S-B-1}}{\partial c_{o}}=0 \quad ; \quad \frac{\partial q_{r}^{N-B-2}}{\partial c_{o}}=\frac{1}{4(1-\delta)}>0$, $\frac{\partial q_{r}^{M-B-2}}{\partial c_{o}}=\frac{2 \delta \beta_{o}+\eta_{o} \Delta_{o}}{4\left(2 \delta \beta_{o}(1-\delta)-\left(\delta \eta_{o}^{2}+2 \delta \eta_{o} \Delta_{o}+\Delta_{o}^{2}\right)\right)}>0, \quad \frac{\partial q_{r}^{S-B-2}}{\partial c_{o}}=\frac{2 \delta \beta_{k}+\Delta_{k}\left(\eta_{k}+\Delta_{k}\right)}{4 \beta_{k}(1-\delta)-\left(\eta_{k}+\Delta_{k}\right)^{2}}>0 ; \quad \frac{\partial q_{r}^{N-C}}{\partial c_{o}}=-\frac{1}{4(1+3 \delta)}<0$, $\frac{\partial q_{r}^{M-C}}{\partial c_{o}}=-\frac{\beta_{o}}{2\left(2 \beta_{o}(1+3 \delta)-\left(\eta_{o}-\Delta_{o}\right)^{2}\right)}<0$, and $\frac{\partial q_{r}^{S-C}}{\partial c_{o}}=-\frac{\beta_{k}}{4 \beta_{k}(1+3 \delta)-\left(\eta_{k}-\Delta_{k}\right)^{2}}<0$.

Similarly, we can verify the following sensitivity analysis result of the equilibrium decisions with respect to $c_{r}$. $\frac{\partial \omega^{N-A}}{\partial c_{r}}=\frac{\partial \omega^{M-A}}{\partial c_{r}}=\frac{\partial \omega^{S-A}}{\partial c_{r}}=0 \quad, \quad \frac{\partial \omega^{N-B-1}}{\partial c_{r}}=\frac{1}{\delta}>0 \quad, \quad \frac{\partial \omega^{M-B-1}}{\partial c_{r}}=\frac{2 \beta_{o}-\eta_{o}^{2}}{2 \delta \beta_{o}+\eta_{o} \Delta_{o}}>0$ $\frac{\partial \omega^{S-B-1}}{\partial c_{r}}=\frac{2 \beta_{k} \delta+\eta_{k} \Delta_{k}}{2\left(\beta_{k} \delta^{2}+\Delta_{k}\left(\eta_{k} \delta+\Delta_{k}\right)\right)}>0 \quad ; \quad \frac{\partial \omega^{N-B-2}}{\partial c_{r}}=\frac{1}{2}>0 \quad, \quad \frac{\partial \omega^{M-B-2}}{\partial c_{r}}=\frac{2 \delta \beta_{o}+\eta_{o} \Delta_{o}}{2\left(2 \delta \beta_{o}-\Delta_{o}^{2}\right)}>0$ $\frac{\partial \omega^{S-B-2}}{\partial c_{r}}=\frac{2(1-\delta) \beta_{k}+\eta_{k}\left(\eta_{k}+\Delta_{k}\right)}{4 \beta_{k}(1-\delta)-\left(\eta_{k}+\Delta_{k}\right)^{2}}>0 ; \quad \frac{\partial \omega^{N-C}}{\partial c_{r}}=\frac{\partial \omega^{M-C}}{\partial c_{r}}=-\frac{1}{2}<0$, and $\frac{\partial \omega^{S-C}}{\partial c_{r}}=\frac{2 \beta_{k}(1+3 \delta)-\eta_{k}\left(\eta_{k}-\Delta_{k}\right)}{4 \beta_{k}(1+3 \delta)-\left(\eta_{k}-\Delta_{k}\right)^{2}}<0$.

$$
\frac{\partial q_{n}^{N-A}}{\partial c_{r}}=\frac{\partial q_{n}^{M-A}}{\partial c_{r}}=\frac{\partial q_{n}^{S-A}}{\partial c_{r}}=0 \quad ; \quad \frac{\partial q_{n}^{N-B-1}}{\partial c_{r}}=-\frac{1}{2 \delta}<0 \quad, \quad \frac{\partial q_{n}^{M-B-1}}{\partial c_{r}}=-\frac{\beta_{o}}{2 \delta \beta_{o}+\eta_{o} \Delta_{o}}<0
$$




$$
\begin{gathered}
\frac{\partial q_{n}^{S-B-1}}{\partial c_{r}}=-\frac{2 \beta_{k} \delta+\eta_{k} \Delta_{k}}{4\left(\beta_{k} \delta^{2}+\Delta_{k}\left(\eta_{k} \delta+\Delta_{k}\right)\right)}<0 \quad ; \quad \frac{\partial q_{n}^{N-B-2}}{\partial c_{r}}=\frac{1}{4(1-\delta)}>0 \quad, \quad \frac{\partial q_{n}^{M-B-2}}{\partial c_{r}}=\frac{\beta \delta}{2\left(2 \beta \delta(1-\delta)-\Delta_{n}^{2}\right)^{2}}>0 \quad, \\
\frac{\partial q_{n}^{S-B-2}}{\partial c_{r}}=\frac{\beta_{k}}{4 \beta_{k}(1-\delta)-\left(\eta_{k}+\Delta_{k}\right)^{2}}>0 \quad ; \quad \frac{\partial q_{n}^{N-C}}{\partial c_{r}}=-\frac{1}{4(1+3 \delta)}<0 \quad, \quad \frac{\partial q_{n}^{M-C}}{\partial c_{r}}=-\frac{\beta_{o}}{2\left(2 \beta_{o}(1+3 \delta)-\left(\eta_{o}-\Delta_{o}\right)^{2}\right)}<0 \quad, \quad \text { and } \\
\frac{\partial q_{n}^{S-C}}{\partial c_{r}}=-\frac{\beta_{k}}{4 \beta_{k}(1+3 \delta)-\left(\eta_{k}-\Delta_{k}\right)^{2}}<0 . \\
(3) \quad \frac{\partial q_{r}^{N-A}}{\partial c_{r}}=\frac{\partial q_{r}^{N-B-1}}{\partial c_{r}}=0 \quad, \quad \frac{\partial q_{r}^{M-A}}{\partial c_{r}}=\frac{\partial q_{r}^{M-B-1}}{\partial c_{r}}=0 \quad, \quad \frac{\partial q_{r}^{S-A}}{\partial c_{r}}=\frac{\partial q_{r}^{S-B-1}}{\partial c_{r}}=0 \quad ; \quad \frac{\partial q_{r}^{N-B-2}}{\partial c_{r}}=-\frac{2-\delta}{4(1-\delta)}<0 \quad, \\
\frac{\partial q_{r}^{M-B-2}}{\partial c_{r}}=-\frac{1}{4}\left(\frac{2 \beta_{o}}{2 \delta \beta_{o}-\Delta_{o}^{2}}+\frac{2 \delta \beta_{o}-\eta_{o}^{2}}{2 \delta \beta_{o}(1-\delta)-\left(\delta \eta_{o}^{2}+2 \delta \eta_{o} \Delta_{o}+\Delta_{o}^{2}\right)}\right)<0 \quad, \quad \frac{\partial q_{r}^{S-B-2}}{\partial c_{r}}=-\frac{2 \beta_{k}(2-\delta)-\eta_{k}\left(\eta_{k}+\Delta_{k}\right)}{2 \delta\left(4 \beta_{k}(1-\delta)-\left(\eta_{k}+\Delta_{k}\right)^{2}\right)}<0 \quad ; \\
\frac{\partial q_{r}^{N-C}}{\partial c_{r}}=-\frac{1}{4(1+3 \delta)}<0, \quad \frac{\partial q_{r}^{M-C}}{\partial c_{r}}=-\frac{\beta_{o}}{2\left(2 \beta_{o}(1+3 \delta)-\left(\eta_{o}-\Delta_{o}\right)^{2}\right)}<0, \text { and } \frac{\partial q_{r}^{S-C}}{\partial c_{r}}=-\frac{\beta_{k}}{4 \beta_{k}(1+3 \delta)-\left(\eta_{k}-\Delta_{k}\right)^{2}}<0 .
\end{gathered}
$$

This concludes the proof of Result 2.

\section{Appendix J. Proof of Result 3}

Comparing the equilibrium component wholesale price and the quantities of new and remanufactured products under scenario $M$ and $S$ with those under scenario $N$, one can confirm that:

(1) $\omega^{M-A}-\omega^{N-A}=0, \quad \omega^{M-B-1}-\omega^{N-B-1}=\frac{\eta_{o}\left(\delta \eta_{o}+\Delta_{o}\right)\left(\delta-c_{r}\right)}{\delta\left(2 \beta_{o} \delta+\eta_{o} \Delta_{o}\right)}>0, \quad \omega^{M-B-2}-\omega^{N-B-2}=-\frac{\left(\delta-c_{r}\right) \Delta_{o}\left(\eta_{o}+\Delta_{o}\right)}{2\left(2 \delta \beta_{o}-\Delta_{o}^{2}\right)}<0$, and $\omega^{M-C}-\omega^{N-C}=0$; $\omega^{S-A}-\omega^{N-A}=-\frac{\eta_{k}^{2}\left(1+c_{k}-c_{o}\right)}{2\left(4 \beta_{k}-\eta_{k}^{2}\right)}<0 \quad, \quad \omega^{S-B-1}-\omega^{N-B-1}=\frac{2 \beta_{k} \delta\left(c_{r}-\delta c_{o}\right)+\eta_{k} \Delta_{k}\left(c_{r}+\delta-2 \delta c_{o}\right)+\Delta_{k}^{2}\left(1+c_{k}-c_{o}\right)}{2\left(\beta_{k} \delta^{2}+\Delta_{k}\left(\eta_{k} \delta+\Delta_{k}\right)\right)}-\frac{c_{r}}{\delta}+c_{o}>0$ $\omega^{S-B-2}-\omega^{N-B-2}=-\frac{\left(1-c_{k}-c_{o}+c_{r}-\delta\right)\left(\Delta_{k}-\eta_{k}\right)\left(\Delta_{k}+\eta_{k}\right)}{2\left(4 \beta_{k}(1-\delta)-\left(\Delta_{k}-\eta_{k}\right)^{2}\right)}$, and $\omega^{S-C}-\omega^{N-C}=-\frac{\left(1-c_{k}-c_{o}+c_{r}-\delta\right)\left(\Delta_{k}-\eta_{k}\right)\left(\Delta_{k}+\eta_{k}\right)}{2\left(4 \beta_{k}(1+3 \delta)-\left(\Delta_{k}-\eta_{k}\right)^{2}\right)}$. As such, we have $\omega^{S-B-2}-\omega^{N-B-2}<0$ and $\omega^{S-C}-\omega^{N-C}<0$ if $\Delta_{k}-\eta_{k}>0$; otherwise, $\omega^{S-B-2}-\omega^{N-B-2}>0$ and $\omega^{S-C}-\omega^{N-C}>0$.

$$
q_{n}^{M-A}-q_{n}^{N-A}=\frac{\beta_{o}^{2}\left(1-c_{k}-c_{o}\right)}{4\left(2 \beta_{o}-\eta_{o}^{2}\right)}>0 \quad, \quad q_{n}^{S-A}-q_{n}^{N-A}=\frac{\beta_{k}^{2}\left(1-c_{k}-c_{o}\right)}{4\left(4 \beta_{k}-\eta_{k}^{2}\right)}>0
$$

$q_{n}^{M-B-1}-q_{n}^{N-B-1}=\frac{\eta_{o} \Delta_{o}\left(c_{r}-\delta\right)}{2 \delta\left(2 \delta \beta_{o}+\eta_{o} \Delta_{o}\right)}<0, q_{n}^{S-B-1}-q_{n}^{N-B-1}=\frac{\Delta_{k}\left(c_{r}\left(\delta \eta_{k}+2 \Delta_{k}\right)-\delta\left(\delta \eta_{k}+\left(1+c_{k}+c_{o}\right) \Delta_{k}\right)\right.}{4 \delta\left(\beta_{k} \delta^{2}+\Delta_{k}\left(\eta_{k} \delta+\Delta_{k}\right)\right)}<0 ;$

$q_{n}^{M-B-2}-q_{n}^{N-B-2}=\frac{\left(\eta_{o}+\Delta_{o}\right)\left(\Delta_{o}\left(\delta\left(c_{k}+c_{o}\right)-c_{r}\right)-\delta \eta_{o}\left(1-c_{k}-c_{o}+c_{r}-\delta\right)\right)}{4(1-\delta)\left(2 \delta \beta_{o}(1-\delta)-\left(\delta \eta_{o}^{2}+2 \delta \eta_{o} \Delta_{o}+\Delta_{o}^{2}\right)\right)}>0 \quad, \quad q_{n}^{S-B-2}-q_{n}^{N-B-2}=\frac{\left(1-c_{k}-c_{o}+c_{r}-\delta\right)\left(\eta_{k}+\Delta_{k}\right)^{2}}{4(1-\delta)\left(4 \beta_{k}(1-\delta)-\left(\eta_{k}+\Delta_{k}\right)^{2}\right)}>0 \quad ;$

$q_{n}^{M-C}-q_{n}^{N-C}=\frac{\left(1-c_{k}-c_{o}-c_{r}+\delta\right)\left(\Delta_{o}-\eta_{o}\right)^{2}}{4(1+3 \delta)\left(2 \beta_{o}(1+3 \delta)-\left(\Delta_{o}-\eta_{o}\right)^{2}\right)}>0$, and $q_{n}^{S-C}-q_{n}^{N-C}=\frac{\left(1-c_{k}-c_{o}-c_{r}+\delta\right)\left(\Delta_{k}-\eta_{k}\right)^{2}}{4(1+3 \delta)\left(4 \beta_{k}(1+3 \delta)-\left(\Delta_{k}-\eta_{k}\right)^{2}\right)}>0$.

(3) $q_{r}^{M-B-2}-q_{r}^{N-B-2}=\frac{1}{4}\left(\frac{2 \beta_{o}\left(\delta-c_{r}\right)}{2 \delta \beta_{o}-\Delta_{o}^{2}}-\frac{c_{r}\left(2 \beta_{o}-\eta_{o}{ }^{2}\right)+\delta \eta_{o}{ }^{2}-2 \delta \beta_{o}\left(c_{k}+c_{o}\right)+\eta_{o} \Delta_{o}\left(1-c_{k}-c_{o}\right)}{2 \delta \beta_{o}(1-\delta)-\left(\delta \eta_{o}{ }^{2}+2 \delta \eta_{o} \Delta_{o}+\Delta_{o}^{2}\right)}\right)-\frac{\left(1+c_{k}+c_{o}+c_{r}\right) \delta-2 c_{r}-\delta^{2}}{4 \delta(1-\delta)}<0$, $q_{r}^{S-B-2}-q_{r}^{N-B-2}=-\frac{\left(1-c_{k}-c_{o}+c_{r}-\delta\right)\left(\eta_{k}+\Delta_{k}\right)\left(\delta \eta_{k}+(2+\delta) \Delta_{k}\right)}{4 \delta(1-\delta)\left(4 \beta_{k}(1-\delta)-\left(\eta_{k}+\Delta_{k}\right)^{2}\right)}<0 ; \quad q_{r}^{M-C}-q_{r}^{N-C}=\frac{\left(1-c_{k}-c_{o}-c_{r}+\delta\right)\left(\Delta_{o}-\eta_{o}\right)^{2}}{4(1+3 \delta)\left(2 \beta_{o}(1+3 \delta)-\left(\Delta_{o}-\eta_{o}\right)^{2}\right)}>0$, and $q_{r}^{S-C}-q_{r}^{N-C}=\frac{\left(1-c_{k}-c_{o}-c_{r}+\delta\right)\left(\Delta_{k}-\eta_{k}\right)^{2}}{4(1+3 \delta)\left(4 \beta_{k}(1+3 \delta)-\left(\Delta_{k}-\eta_{k}\right)^{2}\right)}>0$.

Result 3 is thus proved. 


\section{Appendix K. Proof of Result 4}

By examining the relevant first order partial derivatives, we confirm the following sensitivity analysis result for the two members' equilibrium profits with respect to $c_{r}$.

$$
\begin{aligned}
& \text { (1) } \frac{\partial \Pi_{S}^{N-A}}{\partial c_{r}}=\frac{\partial \Pi_{M}^{N-A}}{\partial c_{r}}=0, \frac{\partial \Pi_{S}^{M-A}}{\partial c_{r}}=\frac{\partial \Pi_{M}^{M-A}}{\partial c_{r}}=0 \text {, and } \frac{\partial \Pi_{S}^{S-A}}{\partial c_{r}}=\frac{\partial \Pi_{M}^{S-A}}{\partial c_{r}}=0 \text {. } \\
& \text { (2) } \frac{\partial \Pi_{S}^{N-B-1}}{\partial c_{r}}=\frac{\delta\left(1+c_{k}+c_{o}\right)-2 c_{r}}{2 \delta^{2}}>0, \frac{\partial \Pi_{S}^{M-B-1}}{\partial c_{r}}=\frac{\beta_{o}\left(2 c_{r}\left(\eta_{o}^{2}-2 \beta_{o}\right)-2 \eta_{o}^{2} \delta+2 \beta_{o} \delta\left(1+c_{k}+c_{o}\right)-\eta_{o} \Delta_{o}\left(1-c_{k}-c_{o}\right)\right)}{\left(2 \beta_{o} \delta+\eta_{o} \Delta_{o}\right)^{2}}>0 \text {, and } \\
& \frac{\partial \Pi_{S}^{S-B-1}}{\partial c_{r}}=\frac{2 \beta_{k} \delta\left(1+c_{k}+c_{o}\right)-c_{r}\left(4 \beta_{k}-\eta_{k}^{2}\right)-\eta_{k}^{2} \delta-\left(1-c_{k}-c_{o}\right) \eta_{k} \Delta_{k}}{4\left(\beta_{k} \delta^{2}+\Delta_{k}\left(\eta_{k}^{2} \delta+\Delta_{k}\right)\right)}>0 \quad ; \quad \frac{\partial \Pi_{M}^{N-B-1}}{\partial c_{r}}=\frac{c_{r}-\delta}{2 \delta^{2}}<0 \\
& \frac{\partial \Pi_{M}^{M-B-1}}{\partial c_{r}}=-\frac{\beta_{o}\left(2 \beta_{o}-\eta_{o}^{2}\right)\left(\delta-c_{r}\right)}{\left(2 \delta \beta_{o}+\eta_{o} \Delta_{o}\right)^{2}}<0 \text {, and } \frac{\partial \Pi_{M}^{S-B-1}}{\partial c_{r}}=-\frac{\left(2 \beta_{k} \delta+\eta_{k} \Delta_{k}\right)\left(\left(2 \beta_{k} \delta+\eta_{k} \Delta_{k}\right)\left(\delta-c_{r}\right)+\left(1-c_{k}-c_{o}\right) \Delta_{k}{ }^{2}\right)}{8\left(\beta_{k} \delta^{2}+\Delta_{k}\left(\eta_{k}{ }^{2} \delta+\Delta_{k}\right)\right)}<0 . \\
& \text { (3) } \frac{\partial \Pi_{S}^{N-B-2}}{\partial c_{r}}=\frac{1-c_{k}-c_{o}+c_{r}-\delta}{4(1-\delta)}>0, \frac{\partial \Pi_{S}^{M-B-2}}{\partial c_{r}}=\frac{\left(2 \beta_{o} \delta+\eta_{o} \Delta_{o}\right)\left(2 \beta_{o} \delta\left(1-c_{o}-c_{k}+c_{r}-\delta\right)-\Delta_{o}\left(\eta_{o}\left(\delta-c_{r}\right)+\Delta_{o}\left(1-c_{o}-c_{k}\right)\right)\right.}{4\left(2 \beta_{o} \delta-\Delta_{o}^{2}\right)\left(2 \beta_{o} \delta(1-\delta)-\left(\delta \eta_{o}^{2}+2 \delta \eta_{o} \Delta_{o}+\Delta_{o}^{2}\right)\right)}>0 \quad \text {, and } \\
& \frac{\partial \Pi_{S}^{S-B-2}}{\partial c_{r}}=\frac{\beta_{k}\left(1-c_{k}-c_{o}+c_{r}-\delta\right)}{4 \beta_{k}(1-\delta)-\left(\eta_{k}+\Delta_{k}\right)^{2}}>0 ; \quad \frac{\partial \Pi_{M}^{N-B-2}}{\partial c_{r}}=\frac{1}{8}\left(\frac{4 c_{r}}{\delta}-\frac{c_{k}+c_{o}-c_{r}}{1-\delta}-3\right)<0, \quad \frac{\partial \Pi_{M}^{M-B-2}}{\partial c_{r}}=\frac{1}{2}\left(-\frac{\beta_{o}\left(\delta-c_{r}\right)}{2 \beta_{o} \delta-\Delta_{o}^{2}}-\frac{1}{4} q_{r}^{M-B-2}\right)<0, \text { and } \\
& \frac{\partial \Pi_{M}^{S-B-2}}{\partial c_{r}}=-\frac{\left(1-c_{k}-c_{o}\right) \beta_{k}\left(2 \beta_{k}(1-\delta)-\eta_{k}\left(\eta_{k}+\Delta_{k}\right)\right)}{\left(2 \beta_{k}(2-\delta)-\eta_{k}\left(\eta_{k}+\Delta_{k}\right)\right)\left(4 \beta_{k}(1-\delta)-\left(\eta_{k}+\Delta_{k}\right)^{2}\right)}<0 . \\
& \text { (4) } \frac{\partial \Pi_{S}^{N-C}}{\partial c_{r}}=-\frac{1-c_{k}-c_{o}-c_{r}+\delta}{4(1+3 \delta)}<0, \frac{\partial \Pi_{S}^{M-C}}{\partial c_{r}}=-\frac{\beta_{o}\left(1-c_{k}-c_{o}-c_{r}+\delta\right)}{2\left(2 \beta_{o}(1+3 \delta)-\left(\eta_{o}-\Delta_{o}\right)^{2}\right)}<0 \text {, and } \frac{\partial \Pi_{S}^{S-C}}{\partial c_{r}}=-\frac{\beta_{k}\left(1-c_{k}-c_{o}-c_{r}+\delta\right)}{2\left(2 \beta_{k}(1+3 \delta)-\left(\eta_{k}+\Delta_{k}\right)^{2)}\right.}>0 \text {; } \\
& \frac{\partial \Pi_{M}^{N-C}}{\partial c_{r}}=-\frac{1-c_{k}-c_{o}-c_{r}+\delta}{8(1+3 \delta)}<0, \frac{\partial \Pi_{M}^{M-C}}{\partial c_{r}}=-\frac{\beta_{o}\left(1-c_{k}-c_{o}-c_{r}+\delta\right)}{4\left(2 \beta_{o}(1+3 \delta)-\left(\eta_{o}-\Delta_{o}\right)^{2}\right)}<0 \text {, and } \frac{\partial \Pi_{M}^{S-C}}{\partial c_{r}}=-\frac{2 \beta_{k}^{2}\left(1-c_{k}-c_{o}-c_{r}+\delta\right)(1+3 \delta)}{\left(4 \beta_{k}(1+3 \delta)-\left(\eta_{k}-\Delta_{k}\right)^{2}\right)^{2}}<0 \text {. }
\end{aligned}
$$

Result 4 is thus proved.

\section{Appendix L. Proof of Result 5}

By examining the relevant first order partial derivatives, one derives the following sensitivity analysis result for the total environmental impact of both new and remanufactured products with respect to $c_{r}$.

(1) $\frac{\partial E^{N-A}}{\partial c_{r}}=\frac{\partial E^{S-A}}{\partial c_{r}}=\frac{\partial E^{M-A}}{\partial c_{r}}=0 \quad ; \quad \frac{\partial E^{N-B-1}}{\partial c_{r}}=-\frac{e_{n}}{2 \delta}<0 \quad, \quad \frac{\partial E^{S-B-1}}{\partial c_{r}}=-\frac{e_{n}\left(2 \beta_{k} \delta+\eta_{k} \Delta_{k}\right)}{4\left(\beta_{k} \delta^{2}+\Delta_{k}\left(\eta_{k} \delta+\Delta_{k}\right)\right)}<0 \quad$ and $\frac{\partial E^{M-B-1}}{\partial c_{r}}=-\frac{e_{n} \beta_{o}}{2 \beta_{o} \delta+\eta_{o} \Delta_{o}}<0 \quad ; \quad \frac{\partial E^{N-C}}{\partial c_{r}}=-\frac{e_{n}+e_{r}}{4(1+3 \delta)}<0 \quad, \quad \frac{\partial E^{S-C}}{\partial c_{r}}=-\frac{\left(e_{n}+e_{r}\right) \beta_{k}}{4 \beta_{k}(1+3 \delta)-\left(\eta_{k}-\Delta_{k}\right)^{2}}<0 \quad, \quad$ and $\frac{\partial E^{M-C}}{\partial c_{r}}=-\frac{\left(e_{n}+e_{r}\right) \beta_{o}}{2\left(2 \beta_{o}(1+3 \delta)-\left(\eta_{o}-\Delta_{o}\right)^{2}\right)}<0$.

(2)

$$
\begin{aligned}
& \frac{\partial E^{N-B-2}}{\partial c_{r}}=\frac{\delta\left(e_{n}+e_{r}\right)-2 e_{r}}{4 \delta(1-\delta)} \quad, \quad \frac{\partial E^{S-B-2}}{\partial c_{r}}=\frac{2 \delta e_{n} \beta_{k}-e_{r}\left(2 \beta_{k}(2-\delta)-\eta_{k}\left(\eta_{k}+\Delta_{k}\right)\right)}{2 \delta\left(4 \beta_{k}(1-\delta)-\left(\eta_{k}+\Delta_{k}\right)^{2}\right)} \\
& \left.\frac{e_{r}\left(\eta_{o}^{2}-2 \beta_{o}\right)+e_{n}\left(2 \beta_{o} \delta+\eta_{o} \Delta_{o}\right)}{2 \delta \beta_{o}(1+\delta)-\delta \eta_{o}^{2}-2 \delta h_{o} \Delta_{o}-\Delta_{o}{ }^{2}}-\frac{2 e_{r} \beta_{o}}{2 \delta \beta_{o}-\Delta_{o}{ }^{2}}\right) .
\end{aligned}
$$

and

$\frac{\partial E^{M-B-2}}{\partial c_{r}}=\frac{1}{4}\left(\frac{e_{r}\left(\eta_{o}^{2}-2 \beta_{o}\right)+e_{n}\left(2 \beta_{o} \delta+\eta_{o} \Delta_{o}\right)}{2 \delta \beta_{o}(1+\delta)-\delta \eta_{o}^{2}-2 \delta h_{o} \Delta_{o}-\Delta_{o}^{2}}-\frac{2 e_{r} \beta_{o}}{2 \delta \beta_{o}-\Delta_{o}^{2}}\right)$.

When the manufacturer remanufactures part of used products (case $h-B-2)$ under scenario $N$, if $\frac{e_{n}}{e_{r}}>\frac{2-\delta}{\delta}$, $\frac{\partial E^{N-B-2}}{\partial c_{r}}>0$; otherwise, $\frac{\partial E^{N-B-2}}{\partial c_{r}}<0$. Under scenario $S$, if $\frac{e_{n}}{e_{r}}>\frac{2 \beta_{k}(2-\delta)-\eta_{k}\left(\eta_{k}+\Delta_{k}\right)}{2 \beta_{k} \delta}, \frac{\partial E^{S-B-2}}{\partial c_{r}}>0$; otherwise, $\frac{\partial E^{S-B-2}}{\partial c_{r}}<0$. Under scenario $M$, if $\frac{e_{n}}{e_{r}}>\frac{2\left(2 \beta_{o}-\eta_{o}{ }^{2}\right)}{2 \beta_{o} \delta+\eta_{o} \Delta_{o}}-\frac{2 \beta_{o} \delta+\eta_{o} \Delta_{o}}{2 \beta_{o} \delta-\Delta_{o}{ }^{2}}, \frac{\partial E^{M-B-2}}{\partial c_{r}}>0$; otherwise, $\frac{\partial E^{M-B-2}}{\partial c_{r}}<0$. Since 
$\frac{2-\delta}{\delta}>\frac{2 \beta_{k}(2-\delta)-\eta_{k}\left(\eta_{k}+\Delta_{k}\right)}{2 \beta_{k} \delta}$ and $\frac{2-\delta}{\delta}>\frac{2\left(2 \beta_{o}-\eta_{o}{ }^{2}\right)}{2 \beta_{o} \delta+\eta_{o} \Delta_{o}}-\frac{2 \beta_{o} \delta+\eta_{o} \Delta_{o}}{2 \beta_{o} \delta-\Delta_{o}{ }^{2}}$, we can infer that $\frac{\partial E^{h-B-2}}{\partial c_{r}}>0 \quad(h \in\{N, M, S\})$ if $\frac{e_{n}}{e_{r}}>\frac{2-\delta}{\delta}$

Result 5 is thus proved.

\section{Appendix M. Proof of Result 6}

Comparing the total environmental impact under scenario $M$ and $S$ with that under scenario $N$, one can confirm that:

$E^{M-A}-E^{N-A}=\frac{\beta_{o}^{2}\left(1-c_{k}-c_{o}\right) e_{n}}{4\left(2 \beta_{o}-\eta_{o}{ }^{2}\right)}>0 \quad, \quad E^{S-A}-E^{N-A}=\frac{\beta_{k}^{2}\left(1-c_{k}-c_{o}\right) e_{n}}{4\left(4 \beta_{k}-\eta_{k}{ }^{2}\right)}>0 \quad ; \quad E^{M-B-1}-E^{N-B-1}=\frac{\eta_{o} \Delta_{o}\left(c_{r}-\delta\right) e_{n}}{2 \delta\left(2 \delta \beta_{o}+\eta_{o} \Delta_{o}\right)}<0 \quad$, $E^{S-B-1}-E^{N-B-1}=\frac{\left(\Delta_{k}\left(c_{r}\left(\delta \eta_{k}+2 \Delta_{k}\right)-\delta\left(\delta \eta_{k}+\left(1+c_{k}+c_{o}\right) \Delta_{k}\right)\right) e_{n}\right.}{4 \delta\left(\beta_{k} \delta^{2}+\Delta_{k}\left(\eta_{k} \delta+\Delta_{k}\right)\right)}<0 ; E^{M-C}-E^{N-C}=\frac{\left(1-c_{k}-c_{o}-c_{r}+\delta\right)\left(\Delta_{o}-\eta_{o}\right)^{2}\left(e_{n}+e_{r}\right)}{4(1+3 \delta)\left(2 \beta_{o}(1+3 \delta)-\left(\Delta_{o}-\eta_{o}\right)^{2}\right)}>0$, and $E^{S-C}-E^{N-C}=\frac{\left(1-c_{k}-c_{o}-c_{r}+\delta\right)\left(\Delta_{k}-\eta_{k}\right)^{2}\left(e_{n}+e_{r}\right)}{4(1+3 \delta)\left(4 \beta_{k}(1+3 \delta)-\left(\Delta_{k}-\eta_{k}\right)^{2}\right)}>0 \quad ; \quad E^{M-B-2}-E^{N-B-2}=\left(q_{n}^{M-B-2}-q_{n}^{N-B-2}\right) e_{n}+\left(q_{r}^{M-B-2}-q_{r}^{N-B-2}\right) e_{r} \quad$, $E^{S-B-2}-E^{N-B-2}=\frac{\left(1-c_{k}-c_{o}+c_{r}-\delta\right)\left(\eta_{k}+\Delta_{k}\right)\left(e_{n} \delta\left(\eta_{k}+\Delta_{k}\right)-\left((2-\delta) \Delta_{k}+\delta \eta_{k}\right) e_{r}\right)}{4(1-\delta)\left(4 \beta_{k}(1-\delta)-\left(\eta_{k}+\Delta_{k}\right)^{2}\right)}$.

As for $E^{M-B-2}-E^{N-B-2}$, if $\frac{e_{n}}{e_{r}}>\bar{E}_{1}, \quad E^{M-B-2}-E^{N-B-2}>0 ;$ otherwise, $\quad E^{M-B-2}-E^{N-B-2}<0 \quad$ where $\bar{E}_{1}=\frac{(1-\delta)\left(2 \beta_{o} \delta(1-\delta)-\left(\delta \eta_{o}^{2}+2 \delta \eta_{o} \Delta_{o}+\Delta_{o}^{2}\right)\right) \bar{E}_{o}}{\left(\eta_{o}+\Delta_{o}\right)\left(\eta_{o} \delta\left(1-c_{k}+c_{o}+c_{r}-\delta\right)-\Delta_{o}\left(\delta\left(c_{k}+c_{o}\right)-c_{r}\right)\right)}>0$ and $\bar{E}_{o}=1+\frac{\delta\left(c_{k}+c_{o}+c_{r}\right)-2 c_{r}}{(1-\delta) \delta}-\frac{2 \beta_{o}\left(\delta-c_{r}\right)}{2 \beta_{o} \delta-\Delta_{o}^{2}}-\frac{2 \beta_{o}\left(\delta\left(c_{k}+c_{o}\right)-c_{r}\right)-\eta_{o}^{2}\left(\delta-c_{r}\right)-\eta_{o} \Delta_{o}\left(1-c_{k}-c_{o}\right)}{\left(2 \beta_{o} \delta(1-\delta)-\left(\delta \eta_{o}^{2}+2 \delta \eta_{o} \Delta_{o}+\Delta_{o}^{2}\right)\right)}$.

As for $E^{S-B-2}-E^{N-B-2}$, if $\frac{e_{n}}{e_{r}}>\bar{E}_{2}$, then $e_{n} \delta\left(\eta_{k}+\Delta_{k}\right)-\left((2-\delta) \Delta_{k}+\delta \eta_{k}\right) e_{r}>0$, subsequently, $E^{S-B-2}-E^{N-B-2}>0$ can be inferred; otherwise, $E^{S-B-2}-E^{N-B-2}<0$, where $\bar{E}_{2}=\frac{(2-\delta) \Delta_{k}+\delta \eta_{k}}{\delta\left(\eta_{k}+\Delta_{k}\right)}>1$.

In summary, if $\frac{e_{n}}{e_{r}}>\max \left\{\bar{E}_{1}, \bar{E}_{2}\right\}, \quad E^{M-B-2}-E^{N-B-2}>0, \quad E^{S-B-2}-E^{N-B-2}>0 \quad ; \quad$ if $\quad 1<\frac{e_{n}}{e_{r}}<\min \left\{\bar{E}_{1}, \bar{E}_{2}\right\} \quad$, $E^{M-B-2}-E^{N-B-2}<0, \quad E^{S-B-2}-E^{N-B-2}<0$.

Result 6 is thus proved. 